\title{
Cultural Tariffs: Appropriation and the Right to Cross Cultural Boundaries *
}

\author{
Abraham Oshotse $^{\dagger}$, Yael Berda ${ }^{\ddagger}$, Amir Goldberg $^{\dagger}$ \\ ${ }^{\dagger}$ Stanford University, ${ }^{\ddagger}$ The Hebrew University of Jerusalem
}

August 11, 2022

\begin{abstract}
Why are some acts of cultural boundary-crossing considered permissible whereas others are repudiated as cultural appropriation? We argue that perceptions of cultural appropriation have formed in response to the emergence of cultural omnivorousness as a dominant form of high-status consumption, making boundary-crossing a source of cultural capital. Consequently, the right to adopt a practice from a culture that is not one's own is determined on the basis of the costs and benefits one is presumed to accrue. People express disapproval at boundary-crossing if they believe that it devalues or extracts value at the expense of the target culture. We call this process cultural tariffing. We test our theory in a between-subject experimental design, demonstrating that those who enjoy a privileged social position, as inferred from their social identity or socioeconomic status, have less normative latitude to cross cultural boundaries. This is explained by perceptions that these actors are either devaluing or exploiting the target culture. While symbolic boundaries and cultural distinction theories are inconsistent with our results, we find that Americans who are disenchanted about group-based social mobility are the ones most likely to be outraged by cultural boundary-crossing. The imposition of a cultural tariff, we argue, is a form of symbolic redistribution.
\end{abstract}

${ }^{*}$ Nofar Gueta provided invaluable research support, and was central to developing some of the early ideas informing this research. We thank Ashley Martin, Dale Miller, Adina Sterling, Brian Lowery, members of the Computational Culture Lab, and participants of the SCANCOR and HBS Negotiations, Organizations \& Markets seminars for their helpful comments. 


\section{Introduction}

Cultural boundaries are becoming increasingly fluid (Bauman 2000; Brubaker 2016). From names to dress codes, practices that once uniquely identified specific social groups are being widely adopted by outsiders. Not all boundaries are equally traversable, however. Caryn Elaine Johnson, for example, an Academy Award winner African-American actress who is professionally known as Whoopi Goldberg, describes herself as Jewish despite being the daughter of a Baptist clergyman and having no Jewish ancestry. "Goldberg is my name," she professed in a 2011 interview, "it's part of my family, part of my heritage. Just like being Black."1 The public's general indifference to Goldberg's identity fluidity stands in contrast to the allegations directed at Hilaria Baldwin, an entrepreneur who is also the spouse of celebrated actor Alec Baldwin, for disingenuously claiming Spanish heritage. Born Hilary Hayward-Thomas in Boston to parents of English, French and German descent, Baldwin also gave her five children Spanish first names. "Their names are after people who were important to me," she said after being accused of cultural appropriation, "they're not names that we pulled out of a hat." 2

Recent years have seen an influx of scandals in which cultural boundary-crossing-from non-Latino white proprietors selling burritos, ${ }^{3}$ to Italian fashion house Gucci's introduction of an Indian Turban as a haute couture accessory ${ }^{4}$ - is repudiated as cultural appropriation. Why is it that some forms of cultural borrowing are seen as unproblematic while others are rejected as appropriation?

Cultures have been borrowing, imitating and adapting each other's practices - from language through cuisine to religious traditions - for millennia (Bhabha 2012; Henrich 2015). The idea that such cross-cultural adoption is a form of unauthorized taking is a fairly recent historical phenomenon. As Figure 1 illustrates, "cultural appropriation" was only introduced into English discourse three decades ago, and has dramatically increased in prevalence since. ${ }^{5}$

[insert Figure 1 about here]

As the term suggests, an act of boundary-crossing is deemed appropriation when the actor adopting the cultural element is seen to be using something she does not rightfully own. The different reactions to Goldberg's and Baldwin's identity traversals suggest, however, that people are not simply primordially averse to all forms of boundary-crossing. Contra 
conventional sociological wisdom, moreover, the license to cross cultural boundaries is not the prerogative of the privileged.

Dominant sociological and social-psychological theories, from symbolic boundaries (Lamont and Molnár 2002) to social identity theory (Tajfel 1982), cannot explain this historical shift. Instead, we integrate two lines of research - one by cultural sociologists and the other by philosophers of aesthetics and arts - to propose a historically contextualized theory of the conditions under which cultural boundary-crossing is perceived as transgressive. Work by cultural sociologists demonstrates that recent decades have seen a shift towards identity fluidity and cultural omnivorousness, making cultural boundary-crossing a source of cosmopolitan capital (Peterson and Kern 1996; Goldberg, Hannan, and Kovács 2016; Friedman and Reeves 2020). People, we contend, consequently understand cultural practices as valuable currency (Schneider 2003; Büyükokutan 2011). The right to adopt a practice from a culture that is not one's own is therefore determined on the basis of the cost one is presumed to have paid, and the benefits one is presumed to have accumulated. Building on work by philosophers (e.g., Tuvel 2021; Matthes 2016; Ziff and Rao 1997), we further argue that people find an act of cultural boundary-crossing transgressive if they believe that it devalues that target culture, or extracts more value than would have accrued to a member of the target culture for performing the same practice. They react disapprovingly to such acts, thereby imposing a cultural tariff on the transgressor.

We test our theoretical predictions in a (preregistered) between-subject set of experiments, demonstrating that acts of cultural boundary-crossing beget negative reactions when the actor is a member of a higher status group, relative to the group whose culture is being adopted, or has relatively high status within her group. Testing the underlying mechanisms producing these reactions, we further demonstrate that people disapprove of such boundarycrossing because they believe that it devalues the target culture or that it extracts value at the expense of the target group. Consistent with our theory, we find that Americans who are disenchanted about group-based social mobility are the ones most likely to respond with outrage to cultural boundary-crossing scenarios. We argue that this relates to their beliefs about redistributive justice. They impose cultural tariffs on high-status individuals, and grant members of disadvantaged groups more license to cross cultural boundaries, as a form of symbolic redistribution. 


\section{Theory}

\section{What is Cultural Appropriation?}

To appropriate something is to take it without permission or authority. In the cultural realm, such permission is conventionally determined on the basis of group membership. Cultural appropriation is therefore defined as an act of taking from a culture that is not one's own (Ziff and Rao 1997). This seemingly straightforward definition is difficult to apply in practice, however, as group membership, cultural uniqueness or even the act of taking itself are ambiguous concepts (Scafidi 2005). Is a North-African immigrant to France who uses African-American lingo and fashion (Beaman 2017) partaking in cultural appropriation or in permissible cultural borrowing? What about a Jewish Israeli serving Arab hummus to her guests (Hirsch 2011)? Scholars have consequently elaborated on this definition, often conditioning appropriation on the power relations between the groups involved (Ziff and Rao 1997), the harm generated by cultural taking (Matthes 2016; Lalonde 2021; Tuvel 2021) or the social and historical context in which this taking occurs (Schneider 2003).

Our intention is not to add to this discussion. Rather, we are concerned with why certain acts of cultural exchange are conventionally perceived as transgressive, whereas others are not. We therefore use the sociological imagery of cultural boundary-crossing (Lamont and Molnár 2002). We refer to the actor doing the boundary-crossing as the adopter, and to members of the group whose culture is being adopted as the target.

A cultural boundary is crossed when two conditions are met. The first requires the adopter to be an outsider to the target culture. This implies that there exists a boundary delineating the target group, and that the adopter is conventionally understood as being outside that boundary. This is not always a trivial determination. The inclusion of Italian musical elements in Mozart's work, for example, can be seen as appropriative or not depending on whether one sees him as Germanic, or as a member of a broader European musical movement (Young 2010, p. 14). We therefore focus on instances in which the adopter and target are separated by an unambiguous social boundary.

Second, the cultural practice being adopted needs to be conventionally associated with the target culture, but not with the adopter's. Hamburgers, for example, may have originated in Germany, but have since become strongly associated with American culture. An American chef serving a hamburger is not crossing a cultural boundary. If, however, the

chef were to garnish this burger with Kimchi - fermented cabbage that is a staple of Korean 
cuisine - a cultural boundary would be crossed. In what follows below, we focus on practices that are strongly and unambiguously associated with a target group.

Two additional definitions are in order. By "group" we mean a collection of people who share a set of unique cultural practices. Group boundaries are often coextensive with other social and cultural dimensions of difference such as race, religion or ethnicity. In fact, some groups intersect multiple such dimensions. In the U.S., for example, Middle-Easterners are often perceived through religious, genealogical or even racial prisms (Maghbouleh, Schachter, and Flores 2022). But a group need not necessarily be delineated along these lines. Gay and lesbian identities, for example, are associated with consistent and distinct cultural practices (Ghaziani 2015). For our purposes, any identity which is conventionally perceived as having distinctive practices qualifies as a target group. Of course, certain dimensions play particularly important roles in organizing social difference. In the U.S., race is an especially potent source of social cleavage. We will return to this important issue when we discuss our results. For the time being, however, we develop our argument in a manner that is abstracted from the specific dimensions of difference that undergird American society.

Second, by "practice" we mean a symbolic expression of group identity, such as cuisine, language or apparel. This definition specifically narrows our focus to expressions that are visible and transmittable, and excludes characteristics - such as skin tone - that are genetically inheritable. It also leaves out what Young (2010) terms object appropriation, namely the taking of physical objects (such as Egyptian mummies displayed in the British Museum). Our attention, instead, is directed at symbols that are freely reproduced. Young (2010) differentiates between two types of such symbolic boundary-crossing. Content boundarycrossing occurs when the adopter incorporates specific symbols, styles or motifs from the target culture into her own behavior. Subject boundary-crossing, in contrast, happens when the adopter represents the target group, for example, when a painter documents members of a culture she does not belong to. This distinction is potentially consequential, as it may affect observers' attributions of intent to the adopter, and we will return to it in our discussion. For the time being, we will develop our argument in general terms, encompassing both content and subject boundary-crossing.

With this conceptual terminology clarified, we can now offer a definition of cultural appropriation: cultural boundary-crossing that is perceived as transgressive. Note that our definition departs from conventional discussions by philosophers and cultural theorists in that we do not aspire to delineate the objective conditions that make an act of cultural 
boundary-crossing appropriative. Rather, taking a sociological approach (Schoon 2022), our definition hinges on the perception of an observer: An act of boundary-crossing is appropriative if observers generally perceive it as such (even if they do not use the language of appropriation to describe it). As various scholars have pointed out (e.g., Büyükokutan 2011; Tuvel 2021), discussions of cultural appropriation often conflate analytical and normative definitions, with the term sometimes used to neutrally connote boundary-crossing in general and other times to connote wrongful instances of cultural exchange. Our nomenclature obviates this confusion. We use boundary-crossing to denote acts of intergroup cultural exchange in general, and appropriation to denote those that are conventionally perceived as transgressive.

People find an act transgressive, and therefore illegitimate, if they believe that it is inconsistent with the rules of the game (Johnson, Dowd, and Ridgeway 2006). As scholars of cultural appropriation have pointed out, boundary-crossing acts are often considered transgressive if they are seen as harmful to the target group (e.g., Matthes 2016; Lalonde 2021). Some acts are inherently more harmful than others. A non-native speaking a foreign language with a purposefully exaggerated accent, for example, is unmistakably ridiculing the target culture. A child mispronouncing a foreign word while learning at school, in contrast, is not. Such differences in the attributes of cultural boundary-crossing acts, and the contexts in which they are enacted, likely explain a significant proportion of the variance in people's reactions. Our focus, however, is the attributes of the adopter, not the act. As we saw earlier, the rules of the game appear to be applied differently to different actors. Holding the act's attributes constant, we therefore ask: When are people normatively licensed to cross cultural boundaries and when are they not?

\section{Sociological Theories of Cultural Boundary-Crossing}

Sociological theory generally makes two broad predictions about the conditions under which an adopter's boundary-crossing behavior is seen as transgressive or permissible. The first argues that people are innately averse to all forms of boundary-crossing. A large body of research demonstrates that behaviors that defy categorical codes breed confusion and dismay (Zuckerman 1999; Goldberg et al. 2016). In particular, acts that breach distinctions between categories of people are often perceived as threats to social order (Zerubavel 1999; Martin 2000; Smångs 2016). This line of work suggests that people are primordialists 
who see cultural categories as natural entities and who therefore universally resist cultural boundary-crossing.

The vast sociological literature on the penalties associated with atypicality has identified a variety of contingencies to the categorical imperative (e.g., Silver, Childress, Lee, Slez, and Dias 2022). This work demonstrates that variation in people's reactions to categorical mixing often stems from their evaluations of actors' identities. They grant high-status individuals more normative latitude to defy categorical codes than they give those lower on the status distribution (Rao, Monin, and Durand 2005). By extension, this would suggest that primordial objections to cultural boundary-crossing would be relaxed the more the adopter enjoys high status.

A second sociological argument, drawing on Bourdieu's (1986) theory of cultural distinction, maintains that members of groups who benefit from the social status quo are the ones most motivated to protect the symbolic boundaries that sustain it. Indeed, from musical consumption (Accominotti, Khan, and Storer 2018) to child naming (Berger and Le Mens 2009), research consistently finds that those enjoying privileged access to resources employ various tactics to symbolically distinguish themselves from members of less advantaged groups. In some domains, such as immigration (Flores and Schachter 2018) or science (Gieryn 1983), group members can rely on legal or institutional instruments to impose boundaries. In the symbolic realm, however, boundary work is mostly performed normatively. Those resisting boundary-crossing do so predominantly by disparaging, socially penalizing and expressing disapproval at acts of perceived boundary transgression.

Distinction theory would therefore predict that repudiations of cultural boundary-crossing as "appropriation" would mostly originate from high-status actors seeking to protect existing social order, and that outrage will ensue when members of low-status groups adopt higher status culture. Members of lower status groups, in contrast, would, by this rationale, have the strongest incentive to partake in cultural mixing that undercuts the symbolic boundaries that sustain their disadvantage.

The reality of contemporary discourse on cultural appropriation, however, appears to be inconsistent both with universal primordialism and high-status distinction. Acts of cultural boundary-crossing are ubiquitous; they are seldom explicitly rejected as appropriation. When such accusations are invoked, they tend to be most commonly directed at high-status individuals. In fact, in a qualitative analysis of recent cultural appropriation scandals reported in the media, we found that the overwhelming majority of cases was 
focused on prominent individuals, typically borrowing cultural elements associated with minority, especially socioeconomically disadvantaged, groups. The various scandals involving the Kardashian-Jenner family, whose members include celebrity business-people and socialites, are instructive. In 2018, for example, the reality TV show star Kim Kardashian was widely repudiated in the media for wearing cornrows, typically associated with the Fulani people of Africa, to the MTV Awards ceremony. Her sister, Kendall Jenner, has repeatedly been criticized on social media for doing the same. More recently, Jenner was widely chastised for culturally appropriating Mexican culture after launching a tequila brand and starring in a commercial promoting it in 2021.

This was not always the case. When Elvis Presley thrust Rock 'n' Roll into mainstream American consciousness in the mid 1950s, for example, he and other white artists like him were mostly not seen as taking something they do not own. ${ }^{6}$ Many, including some Black Rock 'n' Roll singers, saw this racial boundary-crossing as an opportunity to elevate the status of African-American culture (Cook 2004). Opposition to white artists' adoption of this African-American musical genre came predominantly from segregationists who saw it as a threat to their vision of a racially divided America (Altschuler 2003). Incidentally, the primary racialized controversies Presley endured in his time were accusations of racism that an investigation by the popular Black publication Jet magazine failed to find support for (Guralnick 1995). It was only decades later that Elvis, and the rise of Rock 'n' Roll as the dominant popular musical genre of its time, were critically reassessed as a form of cultural appropriation (e.g., Hall 1997).

\section{Omnivorousness and Cultural Appropriation}

The tendency to see certain types of boundary-crossing as appropriative, we argue, can only be fully understood in the context of broader historical processes. The last half century has seen tectonic shifts in Americans' (and Westerners', more broadly) attitudes about the value of ethnic diversity and the desirability of multicultural exchange (Jiménez 2010; Alba and Nee 2005; Joyner and Kao 2005). This period also gave rise to cultural omnivorousness as a dominant form of high-status cultural consumption (Peterson and Kern 1996). Unlike in Bourdieu's mid-twentieth century France, high-status individuals in contemporary Western and industrialized societies exhibit broad and varied cultural tastes. Rather than adhering to categorical boundaries, these affluent, educated and predominantly (though, not exclusively) 
white consumers actively seek out cultural forms that are associated with other, especially lower status groups (Grazian 2005; Johnston and Baumann 2007; Ocejo 2017).

The rise of cultural omnivorousness has led to two important consequences. First, it catalyzed a new mode of cultural commodification. This was especially the case for cultural practices associated with low-status groups. Hitherto seen as valuable only within their respective cultural traditions, these practices suddenly became desirable objects to outsiders. Their perceived authenticity makes them especially appealing (Grazian 2005; Peterson 1999). As Hahl, Zuckerman, and Kim (2017) demonstrate, cultural practices of low-status groups are seen as authentic precisely because they were historically unrecognized as valuable, enabling their high-status adopters to deflect impressions of instrumental status-seeking.

Second, the blurring of cultural boundaries and the concomitant rise of omnivorousness as a form of high-status display altered the meaning of cultural boundary-crossing, making it a form of cultural capital in and of itself. This cultural capital is often directly transferable into economic advantage. As Koppman (2016) shows, for example, omnivorous tastes lead to better employment prospects in creative industries.

Emerging in Western societies during the post-civil rights and countercultural movements of the late nineteen sixties, omnivorousness is, on the one hand, rooted in a multicultural and inclusive zeitgeist (Fishman and Lizardo 2013). At the same time, however, recent work demonstrates that it embodies a logic of distinction that sees cultural breadth as a strategy to maintain high social status (Ollivier 2008; Khan 2012; Friedman and Reeves 2020). Thus, individuals who borrow cultural elements from other social groups are not necessarily doing so to blur the symbolic boundaries separating them. Rather, those with broad cultural tastes are the ones least receptive of atypical cultural products that subvert categorical distinctions (Goldberg et al. 2016). By projecting openness and carrying a cachet of cosmopolitan sophistication, they are reaffirming their exclusive high status.

\section{Devaluation and Extraction}

At the same time that omnivorousness was emerging as a dominant mode of cultural consumption, philosophers and postcolonial scholars began debating cultural appropriation as a moral problem. Even though some remain skeptical that cultural boundary-crossing can be harmful (Young 2010), others have suggested a variety of ways by which it is. Such 
explanations mostly relate to the properties of the act itself, such as when it misrepresents or misrecognizes the target group (Lalonde 2021; Nicholas and Wylie 2012). When such accounts suggest that harm is contingent on the adopter's attributes - irrespective of the nature of the act - they normally make two types of arguments. The first relates harm to the adopter's disregard toward the target culture (Tuvel 2021). The second contends that cultural boundary-crossing is harmful when there exists a power asymmetry between the adopter and target. Arguments about epistemic injustice, for example, find boundarycrossing objectionable when an adopter from a dominant culture borrows from a target group that is already marginalized in public discourse. Even if the target culture is not misrepresented, its portrayal by a high-status outsider reinforces its silencing and marginalization (Matthes 2016).

The vast majority of Americans are likely unaware of these discussions. Even among academics, debates about cultural appropriation have mostly been conducted on the margins; the topic has received little attention from philosophers, for example (Matthes 2016). Although they do not have an explicit theory of cultural appropriation, most Americans observed the changes ushered by the rise of cultural omnivorousness. They intuit, on the one hand, that practices that were once overlooked have become lucrative, and that this value is unequally accrued by high-status actors. Even if they are not conversant in the language of representation or epistemic injustice, they notice that the traversal of a cultural boundary often serves to reinforce, rather than erode, the symbolic distinction between the groups it separates.

How does the crossing of a boundary serve to reinforce it? Building on work by scholars of cultural appropriation, we argue that cultural boundary-crossing reproduces intergroup inequality when the value associated with a cultural practice is not evenly enjoyed on both sides. This occurs through two complementary mechanisms.

We refer to the first as devaluation. Devaluation occurs when boundary-crossing undermines the prestige or reinforces the low status of the target identity. This can happen when actors intentionally parody the target identity, for example, when they mockingly wear a costume for a Halloween party. Yet, devaluation most commonly occurs without explicit derogatory intentions. When white upper-middle class New Yorkers, for example, display tribal African or Native-American art in their otherwise contemporarily designed homes, these artifacts' juxtaposition to Western aesthetics underscores their "primitivity" (Halle 1996). Even when outsiders are explicitly well-intentioned their actions can still devalue the 
target identity. White participants in Brown-Saracino's (2010) study of gentrification, for example, highly value the original multicultural character of the neighborhoods they move into. However, their attempts to protect the neighborhood's authentic nature exoticizes these target identities as nobly savage (Johnston and Baumann 2007).

Second, boundary-crossing can drive a wedge between groups when members of the adopting group derive value that does not accrue to members of the target group. We refer to this mechanism as extraction. In its most simple form, extraction happens when the adopter's social position provides direct economic advantages that members of the target group do not have, such as when a white gallerist selling indigenous art has exclusive access to affluent buyers. Often, the adopter's benefits are more subtly produced. Extraction regularly occurs because the meaning of a borrowed cultural practice becomes reinterpreted by virtue of its enactment by a member of the adopting group. Consider, for example, the white youth in Rodriquez's (2006) study of a local hip-hop scene. Black men participating in hip-hop culture are often associated with the genre's violent connotations. White men, in contrast, consume hip-hop through a colorblind interpretative frame that neutralizes these racially coded and mostly negative meanings. They can be "cool" without having to carry the burden of being Black (Tate 2003). Extraction similarly occurs when the act of boundarycrossing confers benefits only to the adopting party, because of the cultural connotations associated with that identity. The educated middle-class bartenders and butchers in Ocejo's (2017) ethnography, for example, emphasize their handmade production processes. While they can charge a premium for that authenticity, the products of working-class craftspeople following similar procedures are discounted as unsophisticated.

\section{Cultural Tariffs}

It is against this historical backdrop, we contend, that cultural boundary-crossing came to be popularly understood through the prism of ownership and value. People consequently see cultural boundary-crossing as permissible as long as the adopter does not capture more value than the target.

Our argument rests on two assumptions. First, it builds on a large body of work in sociology and social psychology demonstrating that people are driven by a desire for distributive justice. Because recent historical trends rendered previously unlucrative cultural practices valuable commodities, people expect that value to be distributed equally. An act 
of boundary-crossing is perceived as transgressive when it is seen as distirbutively unjust. Second, we assume that people seek to redress this perceived injustice. They do so by expressing disapproval at transgressive boundary-crossing. Such disapproval exacts a normative cost from the transgressor. We refer to this process as the imposition of a cultural tariff.

Imagine two hypothetical social groups, labeled $A$ (the adopting group) and $T$ (the target group), and their respective typical members $a$ and $t$. An act of cultural boundary-crossing occurs when $a$ enacts a cultural practice that is typically associated with group $T$. We argue that such crossing will be perceived as transgressive if the value generated by $a$ is greater than the value accruing to a similar act by $t$, either because of devaluation, extraction, or both. In other words, if $a$ is perceived to be benefiting more or paying less than $t$ from enacting the same cultural practice, this act will be perceived as transgressive. Such a perception will lead the observer to impose a tariff in the form of expressing disapproval. In summary, we predict that:

$$
D: V(a)>V(t)
$$

where $D$ denotes disapproval, and $V(\cdot)$ is the perception of value accrued. This perception is formed either on the basis of the direct costs and benefits associated with the act, or how the act is believed to affect the group's social standing.

In many cases, such as when the adopter sells the products of her cultural boundarycrossing, the value gained is explicitly monetary. Yet, the perceived value need not necessarily be pecuniary. An act of cultural boundary-crossing can generate status by projecting sophistication without directly translating into financial rewards. We assume that observers see value in both types of acts, and treat these as fungible sources of capital (Bourdieu 2018).

Of course, people are not boundary-crossing accountants. They do not impose a tariff that is directly equivalent to the value imbalance between $a$ and $t$. Nevertheless, we contend that the intensity of their disapproval is proportional to their perception of that imbalance. The greater that perception, the stronger the disapproval. This is, ultimately, a moral response rooted in their beliefs about injustice.

\section{Social Position as a Tariff Gauge}

How do people perform these value calculations? Observers likely use various types of information to infer how much value is captured by the adopter. If they are motivated 
by a desire to remedy distributive injustice, then they are most likely to impose cultural tariffs when they believe that value imbalance is caused by the adopter's advantaged social position. We focus on two dimensions of social position: the adopter's social identity and their status position within their group.

The Intergroup Hypothesis. Because boundary-crossing is rooted in a distinction between insiders and outsiders, the differences between their respective groups are especially salient to observers. Observers therefore make inferences about the adopter's costs and benefits on the basis of their understanding of societal intergroup relations and the privileges and disadvantages associated with various group memberships. The greater the perceived power or status difference between groups $A$ and $T$, the less latitude the adopter has to cross the cultural boundary separating the two groups.

In the American multiethnic and multiracial context, the status ordering of various social groups is not always evident, or consensual. Asian-Americans, for example, have experienced high socioeconomic mobility but are still associated with derogatory stereotypes (Lee 2015). We therefore expect the intergroup tariff accounting to apply especially when the status difference between groups is unambiguous. In the US this difference is most pronounced when the adopter is white and the target is Black. In the analyses below we also consider several other social identities that have been historically disadvantaged in the U.S., including Latinos, Native-Americans and Arabs.

HYPOTHESIS 1 (H1): Acts of cultural boundary-crossing will be perceived as transgressive if there exists an unambiguous status difference between the adopter and target that favors the adopter.

The Intragroup Tariff Hypothesis. Systemic advantages, or disadvantages, are not only a product of group identity. Individuals enjoy varying levels of advantage as a function of their socioeconomic position within their group. If our theory of cultural tariffs is correct, then it is likely that observers draw on within-group status as an additional dimension along which they make inferences about the adopter's costs and benefits.

Consider, for example, the musical careers of Eminem and Macklemore, two white American and commercially successful rappers. While Macklemore was raised in an upper-middle class family in Seattle, Eminem grew up in poverty to a single-parenting mother. The latter's life ordeals are commonly featured in his lyrics. Though both singers have expressed 
unequivocal support for racial equality and identification with the African-American community, Eminem is often seen as having legitimate right to this mode of expression whereas Macklemore has more frequently been disparaged as a cultural appropriator. ${ }^{7}$

This dynamic, we contend, embodies a more general principle. Adopters belonging to a high-status group but who occupy a low-status position within that group have more normative latitude to cross boundaries than those occupying a high-status position. The former are seen as having paid a price -in Eminem's case, analogous to the social and economic burden carried by African-American males (Tate 2003) — and as paying authentic homage to the target culture. The latter, on the other hand, are seen as deriving benefits without having suffered a cost. Their advantaged social position confers benefits that their low-status counterparts do not have.

Consider Macklemore again. Though he is clearly aware of his advantaged social positionin fact, one of his earliest songs is titled "White Privilege"- his detractors express doubt about the authenticity of his intentions. They see his homage to Black culture a form of cheap talk that ultimately devalues hip-hop. Macklemore's success with white audiences traditionally outside the scope of hip-hop fans also highlights to his critics his ability, which they attribute to his high socioeconomic background, to access resources mostly unavailable to African-American musicians. ${ }^{8}$

HYPOTHESIS 2 (H2): Acts of cultural boundary-crossing will be perceived as transgressive the higher the adopter's status within her social group.

\section{Mechanisms}

What explains observers' perceptions of transgression? If people were simply averse to the idea of culture being used as a form social currency, they would have objected to all forms of cultural boundary-crossing. We posit, instead, that observers' concern for distributive injustice makes them resistant to cultural boundary-crossing because they believe that the adopter's benefits come at the expense of the target group. This occurs when boundarycrossing devalues the target object, or extracts value that members of the target group are unable to access.

We test the mechanisms of devaluation and extraction in two ways. First, we expect to find that the effects of social identity and socioeconomic status on disapproval (as specified in 
$\mathrm{H} 1$ and $\mathrm{H} 2$, respectively) are mediated by perceptions of devaluation and extraction. As we explain below, we infer these perceptions by asking respondents whether they see boundarycrossing actions as disrespectful and exploitative. We expect to find that respondents' tendency to disapprove cultural boundary-crossing when it is performed by members of a high-status group (H1) or by socioeconomically privileged individuals (H2) is mediated by their perceptions of disrespect and exploitation. Second, we test these mechanisms directly, as we propose in the following two additional hypotheses.

The Devaluation Hypothesis Devaluation occurs when the act of boundary-crossing portrays the target as inferior. This occurs, we contend, when a boundary-crossing act is seen as disrespectful toward the target culture (Tuvel 2021). As Lamont et al (2016) demonstrate, acts of disrespect are perceived as symbolic assaults on the target's worth. They are acts of devaluation. Most boundary-crossing acts, however, are not unequivocally respectful or disrespectful. People therefore rely on their inference about the actor's intent in determining whether the act is respectful or not.

For example, imagine an adopter wearing a tattoo of a symbol, such as the Hindu God Shiva, that is considered sacred in the target culture. This act can be interpreted as devaluation if the symbol is used in a secular manner, implicitly ridiculing the target culture's valorization of the symbol. Alternatively, etching a symbol on one's skin can also be seen as a genuine and costly tribute to the target culture. We argue that observers make inferences about the adopter's intentions of respect, or lack thereof, on the basis of the adopter's connection to the target culture. Adopters who have a history of connection to the target culture have invested a cost in acquainting themselves with that culture and are therefore seen as acting out of intentions of respect. Those who do not have such a history, in contrast, have not paid a cost and are therefore perceived to be devaluing the target culture.

HYPOTHESIS 3 (H3): Acts of cultural boundary-crossing will be perceived as transgressive the more superficial the adopter's connection to the target culture.

The Extraction Hypothesis A second channel leading to disapproval of cultural boundarycrossing is extraction. Extraction occurs when the adopter $a$ derives more value from the act of cultural boundary-crossing than a typical member of the target group, $t$, would derive. As many have pointed out (e.g. Young 2010), cultural appropriation is often experienced by its targets as a form of theft. This subjective sense is especially exacerbated when the adopter 
is able to derive significant value from the act of cultural boundary-crossing, for example, when target cultural forms are commodified and sold at significant profits. These profits are inaccessible to members of the target identity due to structural constraints limiting their ability to translate cultural capital into economic capital (Tate 2003). Thus, members of the adopting group capture the value that was created, through hard work, by members of the target group. We therefore hypothesize:

HYPOTHESIS 4 (H4): Acts of cultural boundary-crossing will be perceived as transgressive the more value derived by the adopter relative to members of the target group.

\section{Moderators}

The Mobility Disenchantment Hypothesis Why do people impose cultural tariffs? Our theory argues that individuals are offended by cultural boundary-crossing because they believe that adopters are unfairly rewarded for cultural capital they do not rightfully own. The protection of cultural boundaries, in other words, is motivated by a desire to redress perceived distributive injustice. Not all Americans share that motivation with equal commitment, however. Indeed, research shows that Americans vary in their perceptions about the magnitude of inequality and their beliefs about its fundamental causes (McCall, Burk, Laperrière, and Richeson 2017). We therefore expect to find that those who subscribe to a worldview that sees American society as systemically unequal should be most resistant to cultural boundary-crossing. They charge cultural tariffs as a form of symbolic redistributive justice.

Sociological work traditionally explores people's perceptions about inequality and redistributive policies in general, irrespective of their perceptions about ethnic and racial relations. Symbolic redistribution, in contrast, is inherently rooted in these intergroup perceptions. The reason that a commercially successful white hip-hop artist causes more discomfort than an equally successful Black country musician, we contend, is that people interpret these artists' market performances in light of the opportunities their different social locations afford.

As many have pointed out, the belief in social mobility - that is, that rewards to effort and talent are independent of one's social background - is a central cultural tenet in American society. Some Americans are more pessimistic than others in their beliefs about 
mobility (McCall 2013). We focus on a variant of mobility pessimism: the belief that ethnic and racial boundaries are primary blockages to mobility and that members of minority groups are structurally disadvantaged in their ability to translate hard work and talent into success. We refer to this sentiment as group-based mobility disenchantment. If cultural tariffs are a means to remediate social injustice then individuals who are disenchanted about mobility should be especially averse to cultural boundary-crossing that is rooted in value imbalance. They will see such acts as inherently unfair, and will be more likely to interpret them as devaluatory and extractive. We therefore hypothesize:

HYPOTHESIS 5 (H5): Disapproval of cultural boundary-crossing (as specified in Hypotheses 1 to 4 above) is moderated by group-based mobility disenchantment.

\section{Research Design}

We employed a between-subject experimental design to explore the hypotheses stated above. Respondents were presented with stimuli describing a hypothetical cultural boundary-crossing scenario, and were asked to provide their reactions. We created 16 scenarios overall, four scenarios for each of the four first hypotheses. Each scenario includes two stimuli: one testing the transgressive condition and another the permissible condition. Each respondent was randomly presented with four scenarios, one per hypothesis, and was randomly assigned to condition within scenario. Scenarios were presented in random order. Our hypotheses and research design were pre-registered with OSF. ${ }^{9}$

For each scenario, respondents were randomly assigned to one of two experimental conditions in which we manipulate a parameter relating to the adopter, the target, or the context in which boundary-crossing is occurring. This manipulation is designed to affect respondents' perceptions of the extent to which the boundary-crossing act is transgressive (per its corresponding hypothesis). We refer to the condition designed to generate heightened perceptions of transgression as the Transgressive Condition, and to the condition designed to elicit muted perceptions of transgression as the Permissible Condition. ${ }^{10}$ We expect the transgressive conditions to generate more disapproval. Respondents' reactions are measured after each stimulus. Figure 2 provides a schematic overview of the experimental procedure. 
[insert Figure 2 about here]

For example, for the scenarios testing the intragroup hypothesis (H2), the adopters in the permissible and the transgressive conditions are, respectively, white individuals of low and high socioeconomic status. An example of such stimuli is illustrated in Figure 3. This scenario depicts a white female who adopts traditional Indian fashion. This female is of high socioeconomic status in the transgressive condition, and of low socioeconomic status in the permissible condition.

[insert Figure 3 about here]

This research design provides two important advantages, above and beyond the ability to identify causal effects with a randomized controlled trial. First, because each respondent evaluates four different scenarios, we can account for fixed between-person differences in the tendency to express disapproval. Indeed, we find that between-person variability alone accounts for $45 \%$ to $48 \%$ in variance in respondent reactions, suggesting that people vary significantly in the extent to which they disapprove boundary-crossing in general. Second, because each hypothesis is tested using four different scenarios, we can include scenario fixed effects in our models. This enables us to ensure that our results are robust to specific operationalization decisions, as we discuss below.

\section{Stimuli}

\section{Scenarios}

The various stimuli we use directly manipulate attributes related to the hypothesis being tested, while keeping other attributes of the cultural boundary-crossing act constant across conditions. We used four scenarios for each hypothesis (numbered with a digit referencing the hypothesis and a letter referencing their sequential order). These scenarios are summarized in Table 1 and are presented in full in Appendix A.

[insert Table 1 about here] 
Stimuli related to the intergroup hypothesis (H1) manipulate the identity of the target group, the adopter group, or both. In all of these scenarios the high status group is white and the low status group is Black. To test the intragroup hypothesis (H2), we manipulated the relative socioeconomic status of a white person adopting a minority practice. We manipulate the perceived authentic connection of a white adopter to a minority group in the stimuli used to test the devaluation hypothesis (H3). Finally, in the stimuli used to test the extraction hypothesis (H4) we manipulate the value derived to a white adopter. We discuss these different scenarios in detail when reviewing the results.

\section{Variability in Acts of Boundary-Crossing}

Our objective is to test the extent to which some people have more latitude to cross cultural boundaries than others, holding the type of boundary-crossing act constant. Nevertheless, we recognize that different types of boundary-crossing acts might generate more or less disapproval. Though this source of variation is outside the scope of our theory, we vary the types of stimuli that we use. Our purpose is not to identify the effects of that variance on observers' disapproval but rather to account for this variance in testing our hypotheses.

We address four dimensions of variability in acts of boundary-crossing. First, we distinguish between recombinant and mimetic acts. Recombinant acts, such as fusion cuisine, mix elements from multiple cultural identities, whereas mimetic acts, such as a non-Latino proprietor operating a taco truck, copy a cultural practice in its entirety. Recombinant acts cross boundaries through the act's atypicality while mimetic acts do so through the variety of practices adopted by the actor. As Goldberg et al. (2016) demonstrate, the former subvert boundaries, and tend to be resisted especially by high-status individuals.

Second, drawing on Young's (2010) nomenclature, we distinguish between subject boundarycrossing in which the adopter represents the target, and content boundary-crossing in which the adopter adopts elements of the target culture. Subject boundary-crossing maintains a distance between the adopter and target. An adopter engaging in content boundarycrossing, in contrast, often implicitly makes a claim to that identity as (at least partially) her own. Both types of boundary-crossing can result in devaluation or extraction. We therefore see no a-priori reason why one should inherently generate greater disapproval than the other.

Third, we distinguish between ephemeral and permanent acts of boundary-crossing. Wearing a party costume, for example, is ephemeral, while naming a child or bodily modifi- 
cation with a tattoo are permanent (even if reversible). We assume that the latter indicate, ceteris paribus, greater respect for the target culture and should therefore, on average, generate less disapproval.

Finally, it is likely that observers differentiate between instances in which the adopter engages in boundary-crossing for commercial reasons versus instances in which the rewards to boundary-crossing are social. We assume that profit-oriented scenarios are more likely to generate disapproval as they are unequivocally instrumental. Nevertheless, we expect the dynamics of cultural tariffing to operate whether the adopter is explicitly engaged in commercial activity or in behavior that generates social currency. The latter can also vary in instrumentality. A public display of boundary-crossing musical preferences, for example, is more likely to project status than private musical consumption.

We do not hypothesize about or directly test the effects of any of these dimensions on observers' disapproval. Rather, we introduce variation along these dimensions in our scenarios (non-systematically), as detailed in table 1. This allows us to rule out the possibility that the effects reported below are exclusively driven by attributes related to the act rather than the actor.

\section{Variables}

Respondents were asked to provide four types of reactions, each scaled on a 5-point Likert scale, ranging from "Not at all" to "Extremely" (see Appendix A for details).

Disapproval. The first two, which are the study's dependent variables, relate to respondents' general disapproval of the adopter's actions. People approve others' behaviors as long as they comply with the "rules of the game," whether they truly believe in these rules or merely assume they are accepted by most others (Johnson et al. 2006). We therefore asked respondents both about their own sense of dismay, as well as their perception of behavioral appropriateness. First, we asked whether the scenario made the respondent feel upset, gauging their personal emotional reaction. Second, we asked whether the behavior described in the scenario was appropriate, gauging the extent to which they believe the behavior is normatively acceptable. These two assessments are related, but distinct. A person can be upset even if she recognizes that a behavior is generally considered permissible, or find the behavior inoffensive even if the behavior is seen by most as inappropriate. For ease of interpretation we reverse-coded the variable appropriate (and labeled it inappropriate). 
An alternative would have been to ask respondents directly whether they perceive an act as cultural appropriation. We did not take this approach for two reasons. First, the term "cultural appropriation" is non-colloquial and therefore unfamiliar to many participants. Using it would have required providing a clear definition. Second, asking directly about cultural appropriation would have primed our participants to see acts as transgressive. Our approach obviates these two disadvantages.

Mediators. Next, we asked for reactions tapping underlying mechanisms that mediate the relationship between experimental condition and disapproval. To gauge devaluation we asked respondents whether the adopter was being respectful to the target identity. To gauge extraction we asked whether the adopter was taking advantage of the target identity. For ease of interpretation we reverse-coded the variable respectful (and labeled it disrespectful). Moderators. To evaluate respondents' group-based mobility disenchantment, we asked them whether they believed that racial minorities are less likely to be successful, compared to whites, even if they are talented and hard-working. Though hypothesis 5 is about cultural identity more broadly, not specifically race, we ask respondents about race given that it is the most dominant dimension of systemic inequality in American society. Our intention is to gauge the extent to which respondents perceive American society as unequal, and therefore unjust.

In addition to respondents' perceptions about mobility, we collected a host of respondent attributes, including ideological positions and sociodemographics. Full descriptions of the variables used in the analyses that follow are provided in detail in Appendix A.

\section{Data}

We recruited 2,999 respondents through the online survey platform Prolific. Of these, 359 respondents failed to pass post-hoc attention checks. Their responses were excluded from the analysis. This means that roughly 650 respondents evaluated each scenario (with half assigned to the transgressive condition and the other half to the permissible condition). Our set of respondents embodies a comprehensive cross-section of the U.S. population (Table A2). 


\section{Results}

\section{General Results}

We begin our analysis by examining results in the aggregate. Figure 4, Panel A, reports respondents' overall reactions to the experimental manipulation. The diagram plots coefficients (and their 95\% confidence intervals) estimated from four linear models, one per reaction type, where the reaction is regressed on experimental condition and responses are pooled across all scenarios. These coefficients should be interpreted as the magnitude of the effect of being in the transgressive condition, relative to being in the permissible condition, on respondents' reactions across all scenarios. All models include respondent and scenario fixed effects. These account for differences in respondents' general tendency to express disapproval, as well as differences in the general disapproval generated by each scenario irrespective of experimental condition. Standard errors are clustered by respondent to account for within-person collinearities.

As the bottom two rows of the plot illustrate, the transgressive condition systematically generates more disapproval - namely, it is perceived as more upsetting and more inappropriate - than the permissible condition (both results are significant at $p<0.001$ ). The effects are comparable in size - both correspond to roughly 0.4 of a standard deviation in the outcome. The upper two rows plot the effects of the transgressive condition on respondents' assessments that the adopter is behaving in a disrespectful or exploitative manner. These are the theorized mechanisms mediating the relationship between the experimental condition and respondents' disapproval. As the plot illustrates, respondents perceive significantly greater disrespect and exploitation in the transgressive condition (both results are significant at $p<0.001$ ). These effects are comparable in size to the effects on disapproval, suggesting that respondents are more offended by the transgressive condition because, as we theorize, they perceive it to be more disrespectful and exploitative.

Panel B explores this relationship further. It plots the portions of the effect of being in the transgressive condition on disapproval that are mediated by disrespect and exploitation. ${ }^{11}$ Overall, these mediated effects account for $77 \%$ and $81 \%$ of the overall experimental effect on feeling upset or perceptions of inappropriateness, respectively. In other words, the overwhelming majority of the experimental effect is attributable to respondents' sense that the behavior is either disrespectful or exploitative. As the plot illustrates, disrespect and 
exploitation correspond differently to disapproval: whereas being upset is primarily mediated by exploitation, perceptions of inappropriateness are primarily mediated by disrespect. Respondents are more outraged by exploitation, while being more likely to find disrespectful boundary-crossing normatively unacceptable.

[insert Figure 4 about here]

Figure 5 decomposes these effects by hypothesis. Black dots (and their $95 \%$ confidence intervals) correspond to the effect of being in the transgressive condition, relative to the permissible condition, estimated with a linear model that is restricted to one hypothesis. These models include scenario fixed effects to account for variability in disapproval by scenario (we cannot include respondent fixed effects as each respondent only sees one random scenario per hypothesis). As the four plots illustrates, all of these effects are significant (at $p<0.001$ for all models, except the effect for the intragroup hypothesis on inappropriate, for which $p=0.008$ ), indicating strong and robust support for hypotheses 1 to 4 .

The red and blue dots correspond to the portions of these effects that are mediated, respectively, by disrespect and exploitation. All mediation effects are significant, suggesting that respondents' tendency to disapprove the transgressive condition is explained, at least to some extent, by their perceptions that the act is either disrespectful or exploitative. ${ }^{12}$ These mediation effects shed light on the mechanisms driving respondents' reactions. Several patterns are worth highlighting.

First, as Panel A demonstrates, respondents' disapproval in the intergroup hypothesis (H1) is equally driven by perceptions of disrespect and exploitation. Namely, the adopter's higher group status is being implicitly associated both with an intention to devalue the target culture, and with an ability to extract value that is not accessible to members of the target group. These perceptions explain a little under half of the overall experimental effects. ${ }^{13}$ This suggests that respondents' dismay is also driven by perceptions that are not directly related to their value calculations. Further analyses indicate that respondents' sense of discomfort additionally relates to perceptions that members of the target group experience greater psychological harm by boundary-crossing when their group has lower status. $^{14}$

Second, as Panel B illustrates, a greater portion of the experimental effect is mediated by disrespect and exploitation when we test the intragroup hypothesis (H2), compared to 
the intergroup hypothesis (H1) (though the overall main effects are smaller). The adopter's privileged socioeconomic status more directly invokes value calculations than her advantaged social identity. Exploitation is a particularly strong mediator, suggesting that socioeconomic status is seen more as an indication about whether the adopter is taking advantage of the target group than whether she is disrespecting it. This might explain why artists like Macklemore are often accused of appropriation even if they are aware of their privilege and express strong appreciation of the target culture. Observers are outraged not by these boundary crossers' derogatory or unselfconscious intentions but by the fact that they are reaping rewards without appearing to have paid significant social costs, irrespective of their intentions.

Finally, Panels $\mathrm{C}$ and $\mathrm{D}$ demonstrate that the experimental effects for the devaluation (H3) and extraction (H4) hypotheses are fully mediated by perceptions of disrespect and exploitation, respectively. This is not surprising given that the scenarios for these hypotheses were specifically designed to test the devaluation and extraction mechanisms. At the same time, disrespect and exploitation significantly mediate experimental effects for both hypotheses, suggesting that even if devaluation and extraction are analytically distinct mechanisms, they appear to blend into one another in respondents' subjective experiences. For example, a white pottery artist appropriating Native-American art is seen to be more disrespectful of Native-Americans than a less commercially successful white artist doing exactly the same (scenario 4c). In other words, mere economic success appears to be also interpreted, to some extent, as an indication about the adopter's intentions to devalue the target group.

[insert Figure 5 about here]

\section{Decomposition by Hypothesis}

Figure 6 plots the effects of being in the transgressive condition, relative to the permissible condition, for each individual scenario. Effects were estimated using simple linear models, each regressing disapproval - either being upset or finding the act inappropriate - on experimental condition (similar models estimating experimental effects on perceptions of disrespect and exploitation are reported in Table A6). 
As the four panels of Figure 6 illustrate, the overwhelming majority of coefficients are positive and statistically significant. Of the 16 scenarios, 13 exhibit a statistically significant reaction to the experimental condition for both types of disapproval. For the remaining three, only one scenario - in which parents use a distinctively African-American name for their baby son (3a) - is insignificant for both disapproval variables (nevertheless, the transgressive condition for this stimulus leads to a substantial and significant increase in perceptions of disrespect, as reported in Table A6). Overall, despite the variability in scenarios within hypothesis, these results provide robust support for hypotheses 1 to 4 . Differences in effects between scenarios provide additional insights into the mechanisms producing respondents' reactions. We discuss these now.

\section{[insert Figure 6 about here]}

\section{Intergroup Tariff Hypothesis}

In the scenarios for the intergroup hypothesis we test whether members of disadvantaged groups have more latitude to cross cultural boundaries than members of higher-status groups. Effects are equally substantial and significant for the first three scenarios, as illustrated in panel A of Figure 6. The first scenario, "Singer" (1a), in which a white singer releases a hiphop album in the transgressive condition, and a Black singer releases a country album in the permissible condition, simultaneously manipulates the adopter's identity and the target practice.

To ensure that this effect is not driven by the fact that both the actor and act are manipulated in scenario 1a, in the following two scenarios- "Musician" (1b) and "Alter ego" (1c) — we keep the actor constant across conditions. In the "Musician" scenario, a white classical musician from New England either plays Country (permissible condition) or Blues (transgressive condition) in clubs, whereas in the "Alter-Ego" scenario an AsianAmerican college student adopts either a preppy (permissible condition) or gangsta-rap (transgressive condition) persona. Though the actor is white in the former and AsianAmerican in the latter, effects for both stimuli are near identical in size and are comparable to those produced by scenario 1a. This suggests that what is driving these experimental effects is not the identity of the adopter as much as the identity of the target. Consistent 
with our expectations, respondents express greater disapproval when the target identity is Black.

Moreover, the effects for scenarios $1 \mathrm{~b}$ and $1 \mathrm{c}$ are almost identical in magnitude, even though the latter, in which a college student adopts an accentuated alter ego identity, is more derogatory in nature. Interestingly, the "Alter Ego" stimulus generates a strong sense of exploitation, significantly greater than its effect on perceptions of disrespect (Table A6), suggesting that respondents see the adoption of a Black identity as a source of social value to the adopter. Consistent with our theory, an exaggerated depiction of affluent whites is seen as less outrageous than a comparably exaggerated depiction of inner-city Black culture, presumably because of the former's advantaged social position.

The experimental effects for the final scenario, where the target identity (Mexican) remains constant and the identity of the adopter (white or Black male) is manipulated, are significant but weaker than the first three. This, we conjecture, is related to respondents' general perceptions that Latinos are not as disadvantaged as African-Americans. Moreover, it demonstrates that respondents are more forceful in their negative reactions to boundarycrossing acts that target low-status groups than they are in granting members of these groups more license to cross cultural boundaries.

\section{Intragroup Tariff Hypothesis}

As we saw above, observers impose larger tariffs when members of privileged groups borrow elements from disadvantaged cultures. If they do so to redress distributive injustice, then the adopter's social position within her group - above and beyond advantages conferred by group membership - should be related to her latitude to cross cultural boundaries. The intragroup hypothesis scenarios test this proposition.

All four scenarios testing the intragroup hypothesis maintain the boundary-crossing act constant and manipulate the adopter's identity between an affluent (transgressive condition) and working-class (permissible condition) white individual. As illustrated in panel B of Figure 6, respondents' disapproval increases in the transgressive condition for all scenarios, but effects on perceptions of appropriateness are insignificant when the target identity is not Black (scenarios $2 \mathrm{~b}$ and $2 \mathrm{c}$ ). The two latter scenarios - one in which the adopter wears a typical Indian clothing garment (sari, 2b) and the other in which the adopter wears a typical Arab garment (kaffiyeh, 2c) - generate similar levels of upset, but do not affect perceptions of inappropriateness. 
We conjecture that these two scenarios generate less disapproval overall, relative to scenarios in which the target identity is Black, because respondents associate these identities less strongly with systemic disadvantage and therefore see less value imbalance in their targeting. Though a minority, Indian-Americans enjoy, on average, relatively high socioeconomic status in American society. Arab-Americans, in contrast, are more socioeconomically marginalized, but most Americans are ambivalent about this disadvantage. In fact, some research suggests that Arabs are the most dehumanized minority in the U.S. (Kteily, Bruneau, Waytz, and Cotterill 2015). Consequently, respondents are less protective of boundaries separating these groups' identities, compared to Black identity. At the same time, we also find that respondents in the transgressive condition see significantly more exploitation in the behavior of the white man adopting a kaffiyeh, but not in the behavior of the white woman wearing a sari (Table A6). We interpret this as indication that, despite their ambivalence toward Arab culture, most Americans associate it with systemic disadvantage. Consistent with cultural tariff theory, this leads them to see cultural boundary-crossing that targets Arab identity as more exploitative than one that targets Indian identity.

\section{Devaluation Hypothesis}

Why do observers impose tariffs on high-status adopters? We argue that this is because

they believe that their boundary-crossing generates value imbalance between the adopter and target groups. To explore this directly, we test the two mechanisms - devaluation and extraction - that generate value imbalance. The devaluation hypothesis posits that boundary-crossing is seen as transgressive if it undermines the target culture. This occurs when the adopter is perceived to be acting instrumentally rather than because of an authentic appreciation of the target culture.

To test this proposition, the scenarios testing the devaluation hypothesis manipulate the adopter's perceived connection to the target identity. As illustrated in panel C of Figure 6, three of the four stimuli generate greater outrage and perceptions of inappropriateness when the adopter is seen as less authentically connected to the target group. The first scenario, however - in which a white couple names their baby with a distinctively Black namedoes not produce experimental effects on feeling upset or inappropriateness. We interpret this to be mostly related to the type of act featured in this scenario. The permanence and symbolic significance of a parent's naming decision implies appreciation of the target 
identity; this, we conjecture, offsets outrage or perceptions of inappropriateness generated by the experimental manipulation. ${ }^{15}$

Nevertheless, we find that in the transgressive condition, when the parents of the baby are not connected to Black culture, respondents see their naming decision as substantively more disrespectful of that identity (Table A6). Naming a white boy with a distinctively Black name can devalue Black culture if the multicultural cachet associated with such a name reinforces the name's perceived Blackness. Respondents appear to use information about the parents' connection to Black culture as an indication about whether the naming decision reflects a strong appreciation of Black culture or an uncommitted appropriation.

The remaining three scenarios generate comparable effects on being upset and perceptions of inappropriateness. In the second and third scenarios adopters integrate traditional cultural elements - Jewish and Arab in scenarios 3b and 3c respectively - into a wedding ceremony. In the fourth scenario, where a white female adopts Arab dress, we manipulate the adopter's connection to the target culture with marriage rather than personal history. This scenario generates slightly weaker effects than the two preceding scenarios. We interpret this as indication that, consistent with the devaluation hypothesis, respondents express disapproval on the basis of the actor's perceived authentic connection to the target culture more than her formal marriage right to that culture.

\section{Extraction Hypothesis}

The second mechanism generating value imbalance occurs when the adopter reaps greater rewards than typical members of the target group would have reaped for similar actions. The final set of scenarios directly tests this hypothesis. Their experimental effects are illustrated in panel D of Figure 6. We included respondents' perceptions of the actor's competence

as a control for all models testing the extraction hypothesis and reported in panel D (see also Table 1). We do so because, as we discovered when pretesting stimuli, respondents tend to interpret commercial success as an indication of competence. This attenuated their disapproval for scenario 4b ("Store"). Adding competence as a control did not substantively alter results in the remaining three scenarios. Nevertheless, for consistency we included it as a control in all models testing the extraction hypothesis. We provide additional details in Appendix C.

In the first three scenarios testing the extraction hypothesis the actor, a white individual, remains constant while the value derived from the act of boundary-crossing is manipulated 
across experimental conditions. In the ethnic passing (4a) scenario the value accrued to the adopter, who is a job candidate, is manipulated by the type of job she is applying for and the importance that employers put on diversity. This is the scenario that, irrespective of experimental condition, generates the highest level of disapproval relative to all other scenarios used in our study. ${ }^{16}$ In support of the extraction hypothesis, we find that this scenario affects respondents' perception of exploitation $(\mathrm{p}<0.001)$ but not of disrespect (Table A6). Namely, their increased sense of dismay and inappropriateness is attributable to the fact - as the extraction hypothesis suggests - that the act of boundary-crossing generates value for the adopter. This value is seen as unfairly extracted because the actor does not suffer the societal costs associated with being Latina.

The two following stimuli-"Store" (4b) and "White Potter" (4c)-directly manipulate the amount of economic value derived by the white adopter. In the former, success is attributed to the actor's business connections and in the latter, where it is tied to his status as the "only white potter in Navajo Nation," directly to his identity. Consequently, respondents report a greater increase in perceptions of exploitation in the transgressive condition for scenario $4 \mathrm{c}$ than scenario $4 \mathrm{~b}$ (Table A6). They see the adopter's success as especially exploitative because he derives value, without having to bear the disadvantages associated with being Native-American, that members of that target group are unable to access.

In the final scenario, "Interethnic Potter" (4d), we rule out that the effects we have seen are simply a product of respondents' negative reaction to winner-take-all success. Here the success of the actor remains the same, but his identity varies from white (transgressive condition) to Native-American (permissible condition). Respondents express far greater disapproval of the success of an outsider relative to an insider to the target group, even if the latter enjoys rare and disproportionate rewards. In other words, their disapproval is not merely rooted in a general objection to distributive injustice. Rather, it is driven by an aversion to injustice that is anchored in intergroup inequality.

\section{Who is Sensitive to Cultural Boundary-Crossing?}

Why do respondents react more disapprovingly to the transgressive conditions? Results so far indicate, in support of the devaluation and extraction hypotheses, that people are more outraged by cultural boundary-crossing when they perceive this action as debasing 
or exploitative of the target culture. Is that reaction consistent across the board, or are some people more likely than others to see certain acts of cultural boundary-crossing as generating value imbalance? If our theory of cultural tariffs is correct then this tendency should be linked to people's perceptions of systemic injustice. To explore that, we report the results of moderation analyses, testing susceptibility to the experimental manipulation.

Formally, moderation is estimated with the following model:

$$
D_{i s}=\beta_{0}+\beta_{1} A_{i s}+\beta_{2} M_{i}+\alpha M_{i} \times A_{i s}+\beta_{3} S_{s}+\epsilon_{i s}
$$

where $i$ indexes individual respondents and $s$ indexes scenario, $O_{i s}$ is the respondent's level of disapproval, $M_{i}$ is the moderator in question (a fixed individual trait), $A_{i s}$ is an indicator of whether the respondent was assigned to the transgressive condition for a given scenario, and $S_{s}$ is a scenario fixed effect. Standard errors are clustered by respondent. $D_{i s}$ is a composite measure of disapproval, averaging the respondent's reactions upset and inappropriate. ${ }^{17} \mathrm{We}$ include scenario fixed effects to account for variance in general disapproval generated by each scenario. The interaction term $\alpha$ estimates moderation, namely, differences in susceptibility to the experimental manipulation. Where moderators include multiple levels we estimate individual coefficients for each level rather than treating the variable as continuous.

\section{The Disenchantment Hypothesis}

The disenchantment hypothesis (H5) posits that individuals who are pessimistic about intergroup mobility are more likely to react negatively to cultural boundary-crossing. Such individuals believe that social boundaries remain significant barriers to opportunity in American society. We contend that they are therefore more likely to be cynical about the boundaryeroding effects of cross-cultural borrowing. Given that they see less mobility opportunity in cultural boundary-crossing, we expect them to be more protective of cultural boundaries. To test this hypothesis we estimate the moderating effect of disenchantment on disapproval.

The marginal effects estimated by this model are plotted in Panel A of Figure 7 (see Model 1, Table 2 for details). As the plot illustrates, the tendency to disapprove the transgressive condition increases significantly with mobility disenchantment. While respondents who are not disenchanted (namely, who strongly disagree that there exist group-based barriers to mobility) react to transgressive boundary-crossing insignificantly differently from

their reactions to permissible boundary-crossing, others react with growing disapproval as 
their disenchantment increases. These results provide strong support for the disenchantment hypothesis. ${ }^{18}$

Panel A also illustrates that the overall level of disapproval, irrespective of experimental condition, increases with disenchantment (both lines have a positive slope). Individuals who are pessimistic about mobility are more sensitive to cultural boundary-crossing in general, in addition to being more prone to disapproval when this crossing is transgressive.

Nevertheless, those pessimistic about mobility are lenient toward some types of boundarycrossing. This becomes apparent when we examine the moderation effect of mobility disenchantment separately for the scenarios testing the intergroup hypothesis (H1) in Panel B. Unlike the scenarios used to test the other hypotheses, the target group in the permissible condition for all H1 scenarios is white. As Panel B illustrates, while mobility disenchantment is associated with an increase in disapproval in the transgressive condition for $\mathrm{H} 1$ scenarios, it is not associated with such an increase in the permissible condition (the slope of the blue line is flat). In other words, those who are strongly disenchanted are as offended by cultural boundary-crossing when the target is privileged as those not disenchanted at all. They are unfazed by cultural boundary-crossing if the target is white.

We interpret these results as additional support for cultural tariff theory for two reasons. First, the intergroup hypothesis tests reactions to cultural boundary-crossing when respondents can only infer the adopter's costs and benefits on the basis of her social identity. This is where respondents' perceptions about systemic intergroup inequality should matter most. Indeed, as panel B illustrates, the moderation effect of mobility disenchantment is stronger when respondents only know the adopter's social identity. It is roughly twice as large as the equivalent effect when respondents also have information about the adopter's socioeconomic status, connection to the target group or success, as illustrated in panel C.

Second, while the scenarios testing the intergroup hypothesis portray Black adopters in the permissible conditions, all adopters in scenarios for other hypotheses are white, irrespective of experimental condition. Thus, respondents who are strongly disenchanted tend to disapprove the latter adopters' behaviors even if they are in the permissible condition because they perceive these white adopters as beneficiaries of their group's advantaged position. Respondents who are not disenchanted do not believe that social identity is a source of advantage and therefore express less disapproval of their cultural boundary-crossing.

We note that respondents who are overly optimistic about mobility significantly disapprove transgressive boundary-crossing only when testing the intergroup hypothesis (Panel 
B), but not when other hypotheses are tested (Panel C). This effect is overwhelmingly driven by their sense of inappropriateness, not their personal outrage. ${ }^{19}$ They recognize that intergroup boundary-crossing is normatively frowned upon when there is significant power imbalance between the adopter and target groups, but are not personally upset by such crossing. Because they do not see this as a matter of distributive justice, they do not express greater disapproval in scenarios that directly test value imbalance.

\section{[insert Figure 7 about here]}

Panels D and E of Figure 7 shed further light on the mechanisms undergirding cultural tariffs. As panel D illustrates, experimental effects are significantly moderated by political ideology. With the exception of those identifying as strongly conservative, respondents disapprove transgressive cultural boundary-crossing. Their disapproval grows as they become more liberal. Panel E demonstrates that differences in reactions to cultural boundary-crossing are also generational. This is consistent with our contention that the rise of cultural appropriation as a social problem is a fairly recent historical phenomenon. Respondents 40 or younger express significantly greater disapproval at transgressive cultural boundary-crossing than those born earlier. The former came of age in the late 1990s, or later, when omnivorous commodification was already in full sway.

Table 2 explores the relationships between these moderators. It reports the results of OLS models with multiple moderators, pooled across all respondents and scenarios and including both scenario and respondent fixed effects. These models are estimated as follows:

$$
D_{i s}=\beta_{0}+\beta_{1} A_{i s}+\alpha_{1} M 1_{i} \times A_{i s}+\ldots+\alpha_{n} M n_{i} \times A_{i s}+\beta_{3} S_{s}+\beta_{4} R_{i}+\epsilon_{i s}
$$

where $R_{i}$ are respondent fixed effects and standard errors are clustered by respondent. The $\alpha$ coefficients estimate moderation effect.

Model 1 reports the standalone moderation effect of mobility disenchantment. When political ideology is included in model 2, this effect is attenuated but remains significant. Sensitivity to boundary-crossing, in other words, increases with mobility disenchantment above and beyond one's self-professed strength of political liberalism. Though ideological identification and mobility disenchantment are related ( 0.456 rank correlation in our sample) they are not one and the same. Disapproval of cultural boundary-crossing does not appear to be a mere display of political identity. Model 3, which also includes age as a moderator, 
suggests that younger respondents' tendency to disapprove cultural appropriation is rooted in their pessimism about mobility. Once their beliefs about mobility are accounted for, age is no longer a significant moderator of the experimental effect.

Together, these results paint a multilayered picture that is consistent with our theory of cultural tariffs. Opposition to cultural boundary-crossing is ideological, inhering in people's beliefs about systemic intergroup inequality. These beliefs appear to translate into a tendency to see certain types of cultural boundary-crossing as transgressive especially among younger cohorts. These respondents were socialized into a world in which symbolic boundaries between groups were eroding but, at the same time, boundary-spanning cultural consumption was becoming a source of cultural capital.

[insert Table 2 about here]

\section{Alternative Hypotheses}

The analyses above are consistent with cultural tariff theory. They demonstrate that observers find cultural boundary-crossing transgressive when it leads to value imbalance through devaluation or extraction. Nevertheless, it is possible that people's reactions to cultural boundary-crossing are shaped by alternative mechanisms that are not related to value calculations as specified by the theory. We consider two main alternatives, both of which attribute variation in perceptions of transgression to variation in observers' motivations.

The first, drawing on a broad sociological literature, relates cultural preferences to social status. Such an approach would argue that expressions of disapproval at boundary-crossing are simply displays of high status, especially if high-status individuals are motivated to deflect accusations of their seemingly exploitative or inauthentic omnivorous behavior (Hahl et al. 2017; Friedman and Reeves 2020).

Our results do not support such an argument, however. As many have noted, education is increasingly becoming a marker of status difference in the U.S. (Fischer and Hout 2006). As Panel F of Figure 7 illustrates, the tendency to react negatively to appropriation is not moderated by education. Model 4 of Table 2, which includes education together with other moderators, further supports the contention that education is not driving perceptions of transgression: the effects of educational attainment are weak and insignificant. This is also 
inconsistent with the notion that sensitivity to appropriation is a form of moral absolutism inculcated through higher education (Broćić and Miles 2021). Those with a college degree or higher disapprove of transgressive boundary-crossing as strongly as those without one.

A different alternative hailing from psychology — identity threat theory — posits that people seek to maintain a positive image of their group identity and, specifically, are motivated to protect the group's differentiation from other identities. In its most basic form, this theory predicts that individuals are particularly offended by boundary-crossing when it is targeted at their own group, as it undermines their self-image (Tajfel 1982).

\section{[insert Figure 8 about here]}

We find little evidence in support of this prediction. To test it, we estimate the extent to which respondents are likely to express more disapproval when the scenario portrays their group as the target of cultural boundary-crossing, relative to outsiders to that group. We regress our measure of disapproval on an indicator of whether or not the respondent is a member of the target group, and include both respondent and scenario-condition fixed effects. We include scenario-condition fixed effects because our purpose is not to estimate susceptibility to experimental condition. Rather, we seek to evaluate the extent to which, observing the exact same scenario-condition combination, members of the target group express greater disapproval. As Panel A (blue coefficients) of Figure 8 illustrates, the effects of being in the target group are statistically insignificant. Contra the expectations of identity threat theory, respondents are not more sensitive to boundary-crossing when their group is the target. ${ }^{20} \mathrm{~A}$ variant of identity threat theory predicts that perceived threat will be especially heightened for minority groups, whose identity is chronically subject to scrutiny and derogation. Because members of minority groups frame their identity in contrast to the majority culture, they may be especially motivated to maintain their group's cultural distinctiveness (Mosley and Biernat 2020). This implies that members of minority groups should express greater disapproval when their group is the target of cultural boundarycrossing. We find no evidence for that either, as illustrated in Panel A (red coefficients). Namely, models identical to those reported in blue, but in which the indicator for being the target identity is only applied to members of minority groups (defined as not choosing any identity other than white), reproduce similarly insignificant effects. ${ }^{21}$

Alternatively, minorities' experiences of identity threat might sensitize them more to cultural appropriation in general, irrespective of whether theirs is the target group. To test for 
that possibility, we interact ethnic/racial identity with experimental condition in a scenario fixed effects moderation model that predicts disapproval (eq. 2 with multiple moderators). The estimates produced by this model are reported in Panel B of Figure 8. These indicate that certain minorities are indeed slightly more sensitive to cultural transgressive boundarycrossing than others. Counter-intuitively, however, these are not members of the most systematically disadvantaged groups in American society. Rather, members of socioeconomically successful ethnic minorities - East-Asians, South-Asians and Jews - express more disapproval in reaction to transgressive, relative to permissible, boundary-crossing. Disadvantaged and marginalized minorities-Blacks, Native-Americans and Arabs - are not more likely to express disapproval in light of appropriation than white Americans are. Latinos, quite surprisingly, are even slightly less likely to express disapproval. In fact, when we include indicators of high status or disadvantaged minority status in the within-respondent multi-moderation model (eq. 3), we find that those belonging to disadvantaged minority groups are less likely to express disapproval at cultural appropriation than whites (the omitted category in Table 2, Model 4). The reasons for this pattern are not readily evident. We speculate that they may be related to mid-status conformity pressures that socioeconomically successful minorities are subjected to (Phillips and Zuckerman 2001). Regardless, these results are inconsistent with an identity threat explanation.

\section{Discussion}

Some acts of cultural boundary-crossing are met with more resistance than others. As our results demonstrate, Americans - irrespective of their own cultural backgrounds - are particularly protective of cultural identities that are seen as socially disadvantaged. Some have argued that high-status adopters have less latitude to cross cultural boundaries because they are assumed to have prejudiced intentions (Mosley and Biernat 2020). We showed, in contrast, that people's calculus goes beyond essentialized beliefs about group-based entitlements. A socioeconomically disadvantaged individual has more latitude to cross cultural boundaries than a well-to-do counterpart with the same cultural genealogy. As we demonstrated, this inheres in people's perceptions about the value derived and costs incurred by the adopter, relative to the target group. The more boundary-crossing devalues the target identity or provides the adopter access to rewards that are inaccessible to the target group's members, the more our respondents disapprove it. They are not merely granting passage 
to people on the basis of their social identities. Rather, they are imposing tariffs in light of their interpretations of these cultural passengers' costs and benefits.

We do not know how exactly people perform this mental accounting. Yet, we conjecture that these calculations are invariably grounded in people's understandings of systemic barriers to opportunity and historical intergroup relations. Their offense at the appropriation of Native-American art, for example, may be related to their beliefs about the discrimination that Native-Americans experience in contemporary American society or to their perceptions of Native-Americans as victims of a historical injustice. Reactions to cultural boundary-crossing, in other words, are fundamentally dependent on individuals' shared historical narratives about group-based power relations and inequalities. It is very likely that people translate these beliefs into value calculations in divergent ways, and that they often over- or under-weigh certain costs and benefits inconsistently. Regardless, as we showed, the more they are disenchanted about intergroup mobility, the more they disapprove of cultural boundary-crossing. The imposition of a cultural tariff, in other words, is a form of symbolic redistribution.

While scholars and political activists likely have explicit and structured frameworks for distinguishing cultural appropriation from permissible boundary-crossing, we imagine that most people employ lay and intuitive theories of transgressive boundary-crossing. These are implicit and inarticulable belief systems, what Lizardo (2017) calls "nondeclarative" knowledge. Our respondents may not be consciously aware of devaluation and extraction when they express disapproval at transgressive boundary-crossing. Nevertheless, the fact that mobility disenchantment strongly explains the intensity with which they react negatively to what we term cultural appropriation suggests that their behaviors are motivated by a moral resentment for distributive injustice.

Media portrayals of contemporary cultural debates tend to focus on heated and often exaggerated exchanges. Yet, a discomfort with devaluatory or extractive boundary-crossing is not merely the purview of "woke" college students or radicalized social activists. Indeed, those on the far end of ideological liberalism and mobility disenchantment are far more likely to find cultural boundary-crossing transgressive. But the majority of our respondents, including those identifying as conservative or denying the existence of systemic mobility barriers, find devaluatory or extractive cultural boundary-crossing unpalatable. We do not know whether most, if any, of our respondents would use the language of appropriation to describe such acts of cultural borrowing. Regardless, most respondents subscribe, some more 
reservedly than others, to a worldview that finds such acts inappropriate and upsetting.

\section{The Historical Context of Cultural Tariffs}

Would we have received similar results had we conducted this experiment half a century ago? Most likely not. Unfortunately, we cannot go back in time to test that hypothesis. We nevertheless conjecture that offense at boundary-crossing, motivated by redistributive justice rather than high-status distinction, is the product of recent cultural shifts in American society. Fifty years ago, omnivorous consumption as a form of status display was relatively in its infancy. The term "cultural appropriation" was barely being used (Figure 1). This does not mean that transgressive boundary-crossing did not exist prior to the nineteen seventies, or that people invariably saw such crossing as benign. Yet two important changes occurred in the five decades that have passed since.

First, cultural boundary-crossing was reinterpreted as a form of cultural capital. Consider mainstream American culture's fascination with Native-American imagery. As Deloria (1998) demonstrates, "playing Indian" has always been a mundane way for white Americans to construct their own national identities. Native-Americans mostly found such enactments exclusionary and belittling, but seldom expressed their frustration as it was not broadly recognized as legitimate. By the late twentieth century, in contrast, New Age spiritualists began adopting Native-American practices in admiration of their indigenous communitarianism and environmentalism. Nevertheless, the fruits of such boundary-crossing-both symbolic and commercial — were mostly enjoyed by the adopters.

It was during that period that a second shift was taking shape, as American academics began debating cultural appropriation as a philosophical and historical phenomenon. This new label - "appropriation" - construed boundary-crossing as a form of unauthorized taking. What had hitherto been seen as innocuous was suddenly re-understood as harmful. Our

results suggest that this way of seeing symbolic intergroup relations is gradually pervading the American public. As we showed, dismay at boundary-crossing does not appear to be motivated by a primordial resistance to boundary-crossing nor is it only expressed by those who are the targets or symbolic casualties of cultural appropriation. Moreover, negative reactions to cultural appropriation are particularly strong among younger respondents, those who had come of age during the last quarter century. Though these patterns do not provide unequivocal support for our contention that cultural tariffs are a reaction to postwar 
multiculturalism and the rise of cultural omnivorousness, they are strongly consistent with such a historical account.

\section{Is it Just about Race?}

How observers distinguish between different social groups is central to the dynamics of cultural tariffing. In the U.S., race is the most prominent dimension of social cleavage. It therefore stands to reason that people see group differences primarily through a racial prism. Indeed, perceptions of race and status are inherently interrelated in America: as social groups such as Irish and Italians climbed up the socioeconomic status ladder, for example, they also became increasingly seen as white (Fox and Guglielmo 2012). Moreover, given that people impose cultural tariffs to redress distributive injustice, historical oppressions along racial lines - from slavery to the dispossession of Native-Americans - likely play a role in people's cultural tariffing calculus. Race is an especially potent force of social difference because racial identity is permanent and prominently observable.

Nevertheless, our findings demonstrate that people's disapproval of cultural boundarycrossing is not exclusively related to perceptions of racial difference. In several scenarios (e.g. 2c, 3b-3d), observers resist boundary traversals when the target is either Arab or Jewish, two groups that are predominantly understood through ethnic or religious, but not racial, lenses. While Arabs are sometimes seen as non-white (Maghbouleh et al. 2022), Jews are mostly associated with whiteness (Goldstein 2008). More generally, we find that respondents protect cultural boundaries delineating ethnic and religious groups, as long as the adopter and target occupy a shared social space. In pretesting of various scenarios we found, for example, that people object when white outsiders adopt Amish cultural practices - an ultratraditionalist Anabaptist Christian group - but are indifferent when the adopter is a white American and the target is the Maori people of New Zealand.

Race, class and ethnicity in the U.S. are historically intertwined. We do not know exactly how observers translate these complex relationships into value calculations. Nevertheless, our results suggest that they find cultural boundary-crossing transgressive when they believe that it is rewarded, and that these rewards are not equally shared across both sides of the boundary. This suggests two ways by which the theory of cultural tariffs may extend beyond the specifics of race relations in the U.S.

First, it is likely that cultural tariff dynamics apply in other domains of cultural difference in American society. Recent debates on transsexualism, for example, are often con- 
strued through the prism of value and cost (Brubaker 2016). Indeed, some feminist thinkers see female transgenders as appropriators who enjoy hardly-fought protections afforded to women, without having fully experienced the disadvantages of life as a female (Da Costa 2021). Similarly, the gentrifying classes in New York (Ocejo 2017) and London (Gest 2016) are "rediscovering" old crafts while displacing the working class, both economically and geographically. Cultural tariff theory might even extend to domains of knowledge production such as science. An economist popularizing an old anthropological insight, for example, might be perceived as engaging in appropriation if her use of anthropological knowledge carries a cachet of intellectual sophistication while her ability to reach a broad audience is seen as a product of her discipline's high academic status. These various boundaries crosscut one another in complex ways. How these intersections affect cultural tariffs - for example, does a female economist have more latitude to incorporate anthropological ideas than a male counterpart? - is beyond the scope of our discussion.

Second, if cultural tariffing is not inherently about policing racial boundaries, it would seem reasonable that boundary-crossing is also seen as transgressive in non-American contexts wherein race is less prominent a source of social difference. Previous research demonstrates that cultural omnivorousness has become a prevalent mode of consumption throughout the industrial world (Fishman and Lizardo 2013). Cultural boundary-crossing is not a uniquely American form of high-status display (Friedman and Reeves 2020). Nevertheless, it is not at all evident that non-Americans would react to cultural boundary-crossing in similar ways. The U.S. is a uniquely multicultural society. It also has a specific history of colonialism, slavery and mass immigration. Because emergent norms about cultural appropriation are structured on the basis of this shared historical imagination, they likely have different national manifestations. Whereas cultural fault lines are mostly racial and ethnic in the U.S., they are predominantly religious in many Western European countries (Drouhot 2021; Bail 2008). Nevertheless, these countries often adopt different institutional approaches to citizenship and the resolution of multicultural friction (Beaman 2017; Koopmans and Statham 1999). We expect these divergent structural and institutional arrangements to have significant impact on the international diffusion and local interpretations of cultural tariffs as an ideology of symbolic redistribution. We leave this investigation for future work. 


\section{What Else Explains Variance in Perceived Transgression?}

Cultural appropriation is a product of a tripartite relationship: (a) a boundary, separating the adopter and the target, (b) an act, in which a target cultural practice is enacted by the adopter, and (c) an observer, evaluating whether this boundary-crossing is permissible or transgressive. Our experiment was primarily focused on the fist component: demonstrating how variation in the adopter's or the target's attributes affects observers' disapproval. We also exploited variation along the third component, showing that different observers reach different conclusions about whether an act of boundary-crossing is permissible or transgressive. We found that respondents' beliefs about inequality and economic opportunity are predictive of their tendency to see boundary-crossing as transgressive. Contra prevailing wisdom, we did not find evidence that this tendency is a form of high-status distinction or that it is a straightforward product of identity or distinctiveness threat.

Our research design intentionally did not evaluate the second component: the nature of the boundary-crossing act. It is highly conceivable, however, that variation in the act's attributes can affect both perceptions of devaluation and extraction. The adoption of sacred practices, for example, likely leads to greater disapproval, as it implies the adopter's indifference to the target's devaluation. Similarly, practices that are permanent or that require costly investment are seen as indications that the adopter invested a cost that is enough to offset extractive gains. As Young (2005) illustrates, both content and subject boundary-crossing can cause offense. Neither is inherently more or less transgressive. These and other attributes likely influence observers' impressions of the adopter's intentions or their entitlement to the target culture, and are outside the scope of our theory.

Nevertheless, one dimension of variation is worthy of additional discussion. Whether the adopter is crossing cultural boundaries as a producer or a consumer of culture likely affects observers' reactions. Given that cultural tariffing is about remediating value imbalance, it would seem reasonable that observers are especially disapproving of acts in which the adopter is explicitly seeking monetary gain. Indeed, we generally find that respondents react more negatively to the transgressive conditions in scenarios in which the adopter is commercially producing culture, as opposed to consuming it. Because our experiment was not designed to evaluate the different effects of production and consumption on observers' reactions, we consider this suggestive, rather than conclusive evidence in support of cultural tariff theory. ${ }^{22}$ 
We note, moreover, that respondents similarly react disapprovingly, even if not always as fiercely, in scenarios portraying consumers of culture. We interpret this as evidence that they recognize the non-monetary status rewards associated with boundary-crossing. The different consumption scenarios we used also vary in levels of explicit instrumentality. Scenario 2b, for example, in which a woman wears a sari to "stand out," is explicitly status-seeking. In scenario $1 \mathrm{~d}$, in contrast, in which a man hosts a Mexican-themed party, status-seeking is implied. The fact that transgressive boundary-crossing generates disapproval whether instrumentally is explicit or implicit suggests that, consistent with our theory, respondents' reactions are driven by value calculation even if the adopter is not unequivocally crossing cultural boundaries for status gain.

\section{Distinction, Status and Boundary-Crossing}

Our findings shed light on two broad areas of sociological inquiry, above and beyond the specific dynamics of cultural tariffing. The first is the sociological literature on categorical boundary-spanning. Research in that vein consistently demonstrates that actors that defy categorical codes are penalized, but that those enjoying high status have more normative latitude to cross categorical boundaries (Phillips and Zuckerman 2001; Rao et al. 2005). Our results stand in stark opposition to this general principle. We find that high-status adopters, whether due to their group identity or their relative standing within their group, are met with strong reproach when they cross cultural boundaries.

We do not interpret our findings as a fundamental challenge to the idea that high-status actors have, in general, more leeway to defy categorical expectations. Rather, our results highlight an important scope condition to this basic principle. Previous work finds that highstatus actors are granted more freedom to cross cultural boundaries because such behavior is interpreted as a sign of creativity, whereas it is mostly seen as an indication of incompetence when performed by actors who are lower on the status hierarchy (Durand and Kremp 2015; Sgourev and Althuizen 2014). For such behavior to signal competence, however, observers need to assume that it is intrinsically driven. The rise of cultural omnivorousness, against the backdrop of rising inequality in Western societies, has increased public concern that economic and cultural elites are motivated by extrinsic rewards in their pursuit of lower status culture (Friedman and Reeves 2020). This suggest that prestige acts as a doubleedged sword. It provides high-status actors with the protection to defy boundaries, but can also make their boundary-crossing behavior seem instrumental and therefore illegitimate. 
How can high-status individuals navigate this fickleness? Recent work on cultural boundaries and consumption has debated whether the rise of cultural omnivorousness undermines or extends Bourdieu's theory of cultural distinction. The emergent consensus is that breadth, as opposed to exclusivity, has become a dominant mode of high-status distinction (Ollivier 2008; Khan 2012; Friedman and Reeves 2020). In the age of multiculturalism, high status individuals seek to balance openness with subtle forms of exclusion. Cultural tariffs might provide a novel means to achieve that objective. They can both be a reaction to the dispossession brought by cultural omnivorousness and a distinction strategy by those enjoying its fruits. Our results hint at that possibility. Our respondents who are most committed to imposing cultural tariffs are also those strongly committed to a liberal identity, above and beyond their beliefs in systemic inequality. And while disadvantaged minorities, mostly Latinos, are the least sensitive to transgressive boundary-crossing, socioeconomically successful minorities protect it with the greatest zeal. Further work might identify when the imposition of cultural tariffs is motivated by perceptions of injustice and when it is a status play intended at deflecting accusations of instrumentality.

\section{Symbolic Redistribution}

Second, our findings are relevant for broader discussions about the dynamics of ethnoracial relations in the U.S. Recent years have seen a growing cultural emphasis on diversity in American universities, workplaces and institutions (Bell and Hartmann 2007; Berrey 2015). Conversations about race are increasingly shifting from denials of racial difference to its recognition. Such recognition, however, also introduces frictions. While diversity is acknowledged and celebrated, discussions of racial and ethnic inequality are suppressed (Douds 2021).

Cultural tariffs might be an avenue for such frictions to be smoothed. Rejecting colorblind ideology, explicit reference to cultural appropriation acknowledges racial and ethnic difference, indeed protects it. Moreover, it recognizes race- and ethnicity-based inequality. The differential license to cross cultural boundaries is intended to address these inequalities. Yet this remediation is, ultimately, contained to the symbolic realm, where it does not pose direct threats to the material interests of high-status individuals. 


\section{Conclusion}

Symbolic boundaries play a fundamental role in sustaining the structural barriers that separate different groups, reinforcing the material inequalities between them (Lamont and Molnár 2002). Sociological theory would therefore predict that an ideology emphasizing these boundaries serves the maintenance of group-based inequality. Though motivated by a redistributive impetus, cultural tariffs ideology - ironically, perhaps - appears to be doing precisely that. Even though we did not ask our respondents to describe their reactions in their own words, we imagine that those most disapproving of cultural boundary-crossing would have explained that they are not seeking to undo the erosion of these symbolic boundaries. Rather, given their disappointment from what they see as the failed promises of diversity and the inauthentic inclusiveness of cultural omnivores, they believe that those most negatively impacted by injustice should have the greatest license to cross cultural boundaries. It remains to be seen whether cultural tariffs undermine or exacerbate groupbased inequality, if they have any material impact at all.

\section{Notes}

\footnotetext{
${ }^{1}$ https://www.thejc.com/news/uk/whoopi-goldberg-i-m-jewish-and-i-talk-to-god-1.23000

${ }^{2}$ https://www.nytimes.com/2020/12/30/style/hilaria-baldwin-interview.html

${ }^{3}$ https://www.nydailynews.com/news/national/burrito-shop-closes-accusations-cultural-appropriation-article1.3192942

${ }^{4}$ https://www.washingtonpost.com/nation/2019/05/16/nordstroms-indy-full-turban-gucci-draws-sikh-protests /

${ }^{5}$ Historically, the rise of the term occurred against the backdrop of a formal recognition of culture as an exercisable human right, particularly the right to cultural heritage and integrity of indigenous peoples. The international covenant on Economic, Social and Cultural rights entered into force in 1976 and included the right to cultural heritage. Only in 2005, however, UNESCO adopted the first legally binding international instrument on culture, the Convention on the Protection and Promotion of the Diversity of Cultural Expressions. While there is little evidence that international human rights treaties themselves change public perceptions, recent studies show that international treaties affect domestic policy in democracies because prior international obligations effect political climate (Chilton 2014).

${ }^{6}$ It may very well be the case that earlier Rock 'n' Roll artists who did not experience commercial success resented Elvis and other white artists' ability to reach audiences that they had no access to, or that Black audiences more broadly saw Elvis' success as an unfair gain at the expense of African-American artists. However, unlike the present day, this discontent was very rarely, if ever, expressed in public.

${ }^{7}$ https://www.newyorker.com/magazine/2016/03/07/macklemore-the-hip-hop-villain
} 
${ }^{8}$ What matters is perception rather than empirical fact. Despite his disadvantaged starting point, Eminem has been overall far more commercially successful that Macklemore, including among affluent white audiences.

${ }^{9}$ https://osf.io/tvp4q/?view_only=b2e30046ab8c4d21a7cc2045fb4028d8

${ }^{10}$ We assume that perceptions of transgression are a matter of degree. Thus these conditions should be seen as eliciting stronger or weaker perceptions of transgression.

${ }^{11}$ These effects were estimated by a structural equations model that includes both disrespect and exploitation as mediators. This model includes scenario, but not respondent, fixed effects.

${ }^{12}$ All of these mediation effects are significant at the $p<0.001$ level, except for the effect of disrespect on upset $(p=0.006)$ and inappropriate $(p=0.014)$ for the intragroup (H2) hypothesis.

${ }^{13}$ In additional analyses we find that this partial mediation is consistent across all scenarios used to test the intergroup hypothesis.

${ }^{14}$ We also asked respondents whether the adopter is being "culturally insensitive." We find that this perception explains an additional portion of the experimental effects, above and beyond disrespect and exploitation.

${ }^{15}$ Indeed, we find that this scenario is seen as least offensive on average across all scenarios used in our study, irrespective of experimental condition.

${ }^{16}$ Respondents report, on average, being 1.07 points more upset in response to this scenario relative to the mean level of dismay they express at other scenarios.

${ }^{17} \mathrm{We}$ do so for ease of presentation. The results we present below are mostly replicated if applied to each variable independently.

${ }^{18}$ We reproduce significant moderation effects for mobility disenchantment when estimating separate models for each hypothesis, which we do not report due to space limitations. This suggests that perceptions of systemic inequality do not only motivate objections to intergroup boundary-crossing. They also undergird observers' negative reactions to boundary-crossing when it is done by adopters with high within-group status, or when it devalues or extracts value from the target group.

${ }^{19}$ Marginal effect estimates of upset for respondents who strongly disagree with mobility disenchantment are 1.227 (95\% confidence interval 1.066 to 1.388 ) in the permissible condition and 1.498 (1.332 to 1.663$)$ in the transgressive condition. For inappropriate, these estimates are 2.355 (2.147 to 2.564) and 3.063 (2.853 to 3.274$)$, respectively.

${ }^{20}$ Because our experiment was not designed to directly test identity threat theory, these results should not be seen as a rejection of that theory. Nevertheless, these results are quite robust. In fact, when estimating a scenario fixed effects model that controls for experimental condition, we find that being a member of the target group significantly reduces disapproval. This effect is driven by the fact that white respondents are generally less sensitive than other people to boundary-crossing targeting white identity.

${ }^{21}$ In that respect, our findings are inconsistent with Mosley and Biernat's (2020), who find that Black respondents are more likely to see boundary-crossing as transgressive than white respondents. They attribute this finding to minority respondents' tendency to set a lower threshold for what constitutes racism. We speculate that these discrepant findings relate to the fact that, in their experimental paradigm, Mosley and Biernat explicitly ask respondents whether an actor portrayed in a hypothetical scenario is appropriating 
the target culture. Framing the exercise in the language of appropriation, we suspect, primes respondents to think about cultural boundary-crossing as harmful.

${ }^{22}$ In models with respondent fixed effects we find that the interaction term between the transgressive condition and a dummy representing whether the scenario portrays a producer is 0.254 when the dependent variable is upset and 0.344 when it is inappropriate (both at $p<0.001$ ).

\section{References}

Accominotti, Fabien, Shamus R. Khan, and Adam Storer. 2018. "How Cultural Capital Emerged in Gilded Age America: Musical Purification and Cross-Class Inclusion at the New York Philharmonic." American Journal of Sociology 123:1743-1783.

Alba, Richard and Victor Nee. 2005. Remaking the American Mainstream: Assimilation and Contemporary Immigration. Cambridge, Mass.: Harvard University Press, f first paperback edition used edition.

Altschuler, Glenn C. 2003. All Shook Up: How Rock 'n' Roll Changed America. Oxford University Press.

Bail, Christopher A. 2008. "The Configuration of Symbolic Boundaries against Immigrants in Europe." American Sociological Review 73:37-59.

Bauman, Zygmunt. 2000. Liquid Modernity. Cambridge, UK : Malden, MA: Polity, 1st edition edition.

Beaman, Jean. 2017. Citizen Outsider: Children of North African Immigrants in France. Oakland, California: University of California Press, first edition.

Bell, Joyce M. and Douglas Hartmann. 2007. "Diversity in Everyday Discourse: The Cultural Ambiguities and Consequences of "Happy Talk"." American Sociological Review 72:895914.

Berger, Jonah and Gaël Le Mens. 2009. "How Adoption Speed Affects the Abandonment of Cultural Tastes." Proceedings of the National Academy of Sciences 106:8146-8150.

Berrey, Ellen. 2015. The Enigma of Diversity: The Language of Race and the Limits of Racial Justice. University of Chicago Press. 
Bhabha, Homi K. 2012. The Location of Culture. Routledge.

Bourdieu, Pierre. 1986. Distinction: A Social Critique of the Judgement of Taste. London: Routledge, 1 edition edition.

Bourdieu, Pierre. 2018. "The Forms of Capital." In The Sociology of Economic Life, pp. 78-92. Routledge.

Broćić, Miloš and Andrew Miles. 2021. "College and the "Culture War": Assessing Higher Education's Influence on Moral Attitudes." American Sociological Review 86:856-895.

Brown-Saracino, Japonica. 2010. A Neighborhood That Never Changes: Gentrification, Social Preservation, and the Search for Authenticity. Chicago ; London: University of Chicago Press, illustrated edition edition.

Brubaker, Rogers. 2016. Trans: Gender and Race in an Age of Unsettled Identities. Princeton: Princeton University Press.

Büyükokutan, Barış. 2011. "Toward a Theory of Cultural Appropriation: Buddhism, the Vietnam War, and the Field of U.S. Poetry." American Sociological Review 76:620-639.

Chilton, Adam S. 2014. "The Influence of International Human Rights Agreements on Public Opinion: An Experimental Study." Chicago Journal of International Law 15:110-137.

Cook, Jody. 2004. "Graceland National Historic Landmark Nomination." United States Department of the Interior.

Da Costa, Jade Crimson Rose. 2021. "The "New" White Feminism: Trans-Exclusionary Radical Feminism and the Problem of Biological Determinism in Western Feminist Theory." In TransNarratives: Scholarly and Creative Works on Transgender Experience, edited by Kristi Carter and James Brunton, pp. 317-334. Canadian Scholars' Press.

Deloria, Philip Joseph. 1998. Playing Indian. Yale University Press.

Douds, Kiara Wyndham. 2021. "The Diversity Contract: Constructing Racial Harmony in a Diverse American Suburb." American Journal of Sociology 126:1347-1388.

Drouhot, Lucas G. 2021. "Cracks in the Melting Pot? Religiosity and Assimilation among the Diverse Muslim Population in France." American Journal of Sociology 126:795-851. 
Durand, Rodolphe and Pierre-Antoine Kremp. 2015. "Classical Deviation: Organizational and Individual Status as Antecedents of Conformity." Academy of Management Journal 59:65-89.

Fischer, Claude S. and Michael Hout. 2006. Century of Difference: How America Changed in the Last One Hundred Years. Russell Sage Foundation.

Fishman, Robert M. and Omar Lizardo. 2013. "How Macro-Historical Change Shapes Cultural Taste: Legacies of Democratization in Spain and Portugal." American Sociological Review 78:213-239.

Flores, René D. and Ariela Schachter. 2018. "Who Are the "Illegals"? The Social Construction of Illegality in the United States." American Sociological Review 83:839-868.

Fox, Cybelle and Thomas A. Guglielmo. 2012. "Defining America's Racial Boundaries: Blacks, Mexicans, and European Immigrants, 1890-1945." American Journal of Sociology 118:327-379.

Friedman, Sam and Aaron Reeves. 2020. "From Aristocratic to Ordinary: Shifting Modes of Elite Distinction." American Sociological Review 85:323-350.

Gest, Justin. 2016. The New Minority. New York, NY: Oxford University Press, 1 edition edition.

Ghaziani, Amin. 2015. There Goes the Gayborhood? Princeton University Press, reprint edition edition.

Gieryn, Thomas F. 1983. "Boundary-Work and the Demarcation of Science from NonScience: Strains and Interests in Professional Ideologies of Scientists." American Sociological Review 48:781-795.

Goldberg, Amir, Michael T. Hannan, and Balázs Kovács. 2016. "What Does It Mean to Span Cultural Boundaries? Variety and Atypicality in Cultural Consumption." American Sociological Review 81:215-241.

Goldstein, Eric L. 2008. The Price of Whiteness: Jews, Race, and American Identity. Princeton: Princeton University Press. 
Grazian, David. 2005. Blue Chicago: The Search for Authenticity in Urban Blues Clubs. University of Chicago Press.

Guralnick, Peter. 1995. Last Train to Memphis: The Rise of Elvis Presley. Boston: Back Bay Books.

Hahl, Oliver, Ezra W. Zuckerman, and Minjae Kim. 2017. "Why Elites Love Authentic Lowbrow Culture: Overcoming High-Status Denigration with Outsider Art." American Sociological Review 82:828-856.

Hall, Perry A. 1997. "African-American Music: Dynamics of Appropriation and Innovation." In Borrowed Power: Essays on Cultural Appropriation, edited by Bruce H. Ziff and Pratima V. Rao, pp. 31-51. Rutgers University Press.

Halle, David. 1996. Inside Culture: Art and Class in the American Home. Chicago; London: University of Chicago Press, 1st edition edition.

Henrich, Joseph. 2015. The Secret of Our Success: How Culture Is Driving Human Evolution, Domesticating Our Species, and Making Us Smarter. Princeton: Princeton University Press.

Hirsch, Dafna. 2011. "Hummus Is Best When It Is Fresh and Made by Arabs": The Gourmetization of Hummus in Israel and the Return of the Repressed Arab." American Ethnologist 38:617-630.

Jiménez, Tomás R. 2010. "Affiliative Ethnic Identity: A More Elastic Link between Ethnic Ancestry and Culture." Ethnic and Racial Studies 33:1756-1775.

Johnson, Cathryn, Timothy J. Dowd, and Cecilia L. Ridgeway. 2006. "Legitimacy as a Social Process." Annual Review of Sociology 32:53-78.

Johnston, Josée and Shyon Baumann. 2007. "Democracy versus Distinction: A Study of Omnivorousness in Gourmet Food Writing." American Journal of Sociology 113:165-204.

Joyner, Kara and Grace Kao. 2005. "Interracial Relationships and the Transition to Adulthood." American Sociological Review 70:563-581.

Khan, Shamus Rahman. 2012. Privilege: The Making of an Adolescent Elite at St. Paul's School. Princeton, NJ: Princeton University Press. 
Koopmans, Ruud and Paul Statham. 1999. "Challenging the Liberal Nation-State? Postnationalism, Multiculturalism, and the Collective Claims Making of Migrants and Ethnic Minorities in Britain and Germany." American Journal of Sociology 105:652-696.

Koppman, Sharon. 2016. "Different Like Me: Why Cultural Omnivores Get Creative Jobs." Administrative Science Quarterly 61:291-331.

Kteily, Nour, Emile Bruneau, Adam Waytz, and Sarah Cotterill. 2015. "The Ascent of Man: Theoretical and Empirical Evidence for Blatant Dehumanization." Journal of Personality and Social Psychology 109:901.

Lalonde, Dianne. 2021. "Does Cultural Appropriation Cause Harm?" Politics, Groups, and Identities 9:329-346.

Lamont, Michèle and and Virág Molnár. 2002. "The Study of Boundaries in the Social Sciences." Annual Review of Sociology 28:167-195.

Lamont, Michèle, Graziella Moraes Silva, Jessica Welburn, Joshua Guetzkow, Nissim Mizrachi, Hanna Herzog, and Elisa Reis. 2016. Getting Respect: Responding to Stigma and Discrimination in the United States, Brazil, and Israel. Princeton University Press.

Lee, Jennifer. 2015. "From Undesirable to Marriageable: Hyper-Selectivity and the Racial Mobility of Asian Americans." The ANNALS of the American Academy of Political and Social Science 662:79-93.

Lizardo, Omar. 2017. "Improving Cultural Analysis: Considering Personal Culture in Its Declarative and Nondeclarative Modes." American Sociological Review 82:88-115.

Maghbouleh, Neda, Ariela Schachter, and René D. Flores. 2022. "Middle Eastern and North African Americans May Not Be Perceived, nor Perceive Themselves, to Be White." Proceedings of the National Academy of Sciences 119:e2117940119.

Martin, John Levi. 2000. "What Do Animals Do All Day?: The Division of Labor, Class Bodies, and Totemic Thinking in the Popular Imagination." Poetics 27:195-231.

Matthes, Erich Hatala. 2016. "Cultural Appropriation Without Cultural Essentialism?" Social Theory and Practice 42:343-366. 
McCall, Leslie. 2013. The Undeserving Rich: American Beliefs about Inequality, Opportunity, and Redistribution. Cambridge University Press.

McCall, Leslie, Derek Burk, Marie Laperrière, and Jennifer A. Richeson. 2017. "Exposure to Rising Inequality Shapes Americans' Opportunity Beliefs and Policy Support." Proceedings of the National Academy of Sciences 114:9593-9598.

Mosley, Ariel J. and Monica Biernat. 2020. "The New Identity Theft: Perceptions of Cultural Appropriation in Intergroup Contexts." Journal of Personality and Social Psychology pp. No Pagination Specified-No Pagination Specified.

Nicholas, George P. and Alison Wylie. 2012. "“Do Not Do unto Others...": Cultural Misrecognition and the Harms of Appropriation in an Open-Source World." In Appropriating the Past: Philosophical Perspectives on the Practice of Archaeology, edited by Geoffrey Scarre and Robin Coningham, pp. 195-221. Cambridge: Cambridge University Press.

Ocejo, Richard E. 2017. Masters of Craft: Old Jobs in the New Urban Economy. Princeton University Press.

Ollivier, Michèle. 2008. "Modes of Openness to Cultural Diversity: Humanist, Populist, Practical, and Indifferent." Poetics 36:120-147.

Peterson, Richard A. 1999. Creating Country Music: Fabricating Authenticity. University of Chicago Press.

Peterson, Richard A. and Roger M. Kern. 1996. "Changing Highbrow Taste: From Snob to Omnivore." American Sociological Review 61:900-907.

Phillips, Damon J. and Ezra W. Zuckerman. 2001. "Middle-Status Conformity: Theoretical Restatement and Empirical Demonstration in Two Markets." American Journal of Sociology 107:379-429.

Rao, Hayagreeva, Philippe Monin, and Rodolphe Durand. 2005. "Border Crossing: Bricolage and the Erosion of Categorical Boundaries in French Gastronomy." American Sociological Review 70:968-991.

Rodriquez, Jason. 2006. "Color-Blind Ideology and the Cultural Appropriation of Hip-Hop." Journal of Contemporary Ethnography 35:645-668. 
Scafidi, Susan. 2005. Who Owns Culture?: Appropriation and Authenticity in American Law. New Brunswick, N.J: Rutgers University Press, none ed. edition edition.

Schneider, Arnd. 2003. "On 'Appropriation'. A Critical Reappraisal of the Concept and Its Application in Global Art Practices." Social Anthropology 11:215-229.

Schoon, Eric W. 2022. "Operationalizing Legitimacy." American Sociological Review 87:478503.

Sgourev, Stoyan V. and Niek Althuizen. 2014. "“Notable" or "Not Able": When Are Acts of Inconsistency Rewarded?" American Sociological Review 79:282-302.

Silver, Daniel, Clayton Childress, Monica Lee, Adam Slez, and Fabio Dias. 2022. "Balancing Categorical Conventionality in Music." American Journal of Sociology 128:224-286.

Smångs, Mattias. 2016. "Doing Violence, Making Race: Southern Lynching and White Racial Group Formation." American Journal of Sociology 121:1329-1374.

Tajfel, H. 1982. "Social Psychology of Intergroup Relations." Annual Review of Psychology 33:1-39.

Tate, Greg. 2003. Everything But the Burden: What White People Are Taking from Black Culture. Crown.

Tuvel, Rebecca. 2021. "Putting the Appropriator Back in Cultural Appropriation." The British Journal of Aesthetics 61:353-372.

Young, James O. 2005. "Profound Offense and Cultural Appropriation." The Journal of Aesthetics and Art Criticism 63:135-146.

Young, James O. 2010. Cultural Appropriation and the Arts. John Wiley \& Sons.

Zerubavel, Eviatar. 1999. Social Mindscapes: An Invitation to Cognitive Sociology. Cambridge, MA: Harvard University Press, reprint edition edition.

Ziff, Bruce H. and Pratima V. Rao. 1997. Introduction to Cultural Appropriation: A Framework for Analysis, pp. 1-27. Rutgers University Press.

Zuckerman, Ezra W. 1999. "The Categorical Imperative: Securities Analysts and the Illegitimacy Discount." American Journal of Sociology 104:1398-1438. 
Tables 


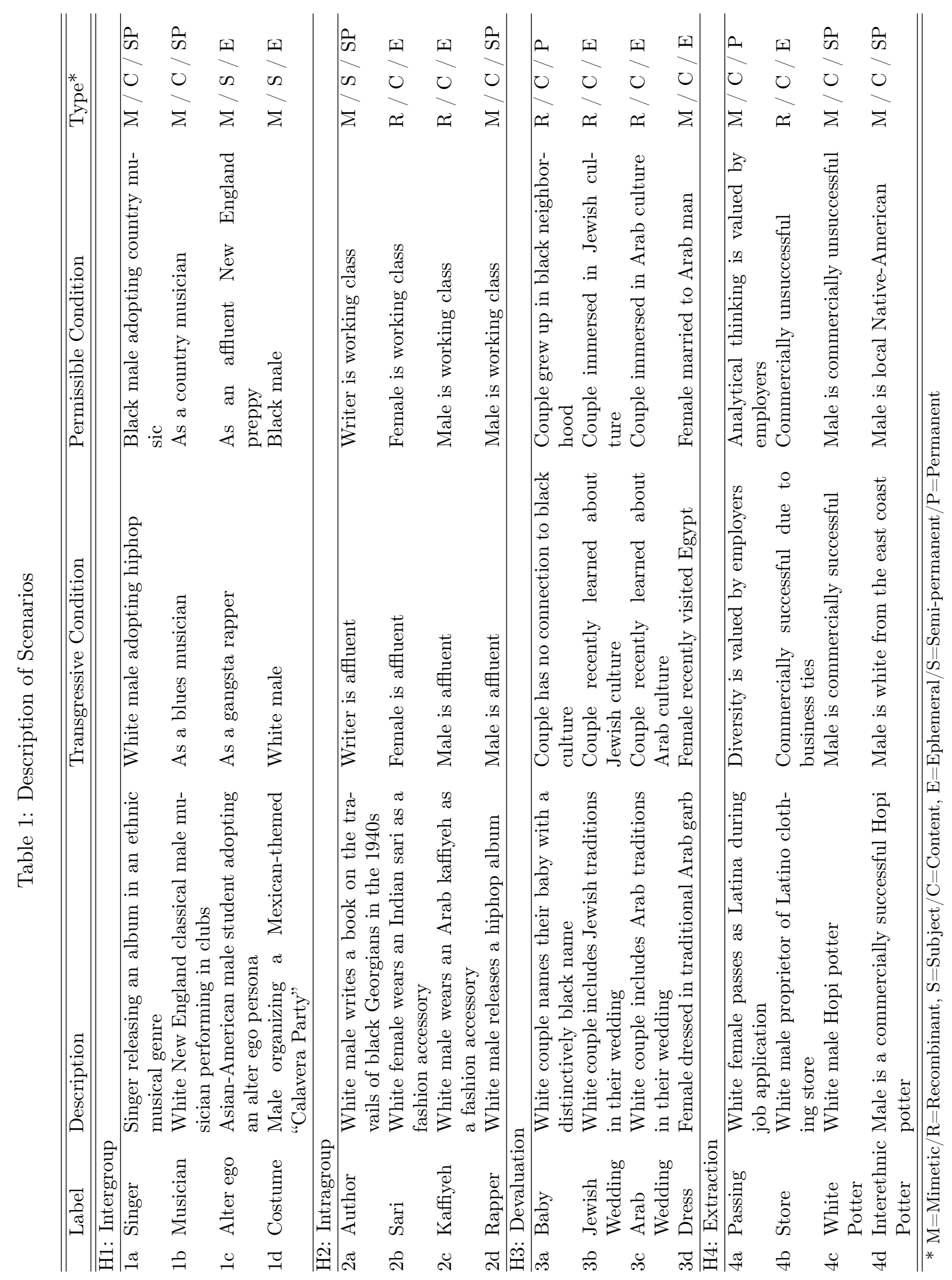


Table 2: OLS Models of Disapproval with Experimental Effect Moderators

\begin{tabular}{|c|c|c|c|c|c|c|c|c|}
\hline & \multicolumn{2}{|c|}{ (1) } & \multicolumn{2}{|c|}{$(2)$} & \multicolumn{2}{|c|}{$(3)$} & \multicolumn{2}{|c|}{$(4)$} \\
\hline Transgressive & $0.247^{* * *}$ & $(0.000)$ & 0.0665 & $(0.683)$ & 0.107 & $(0.514)$ & 0.0818 & $(0.720)$ \\
\hline \multicolumn{9}{|c|}{ Moderators (interactions with transgressive condition) } \\
\hline Disenchantment: & & & & & & & & \\
\hline Disagree & -0.0591 & $(0.466)$ & -0.0828 & $(0.291)$ & -0.0831 & $(0.290)$ & -0.0880 & $(0.262)$ \\
\hline Neither & 0.100 & $(0.219)$ & 0.0525 & $(0.513)$ & 0.0487 & $(0.545)$ & 0.0364 & $(0.651)$ \\
\hline Agree & $0.242^{* *}$ & $(0.001)$ & $0.145^{+}$ & $(0.050)$ & $0.141^{+}$ & $(0.058)$ & $0.124^{+}$ & $(0.095)$ \\
\hline Strongly Agree & $0.466^{* * *}$ & $(0.000)$ & $0.300^{* * *}$ & $(0.001)$ & $0.293^{* * *}$ & $(0.001)$ & $0.275^{* *}$ & $(0.002)$ \\
\hline \multicolumn{9}{|l|}{ Ideology: } \\
\hline Conservative & & & 0.146 & $(0.358)$ & 0.139 & $(0.379)$ & 0.135 & $(0.384)$ \\
\hline Weak Conservative & & & 0.189 & $(0.236)$ & 0.186 & $(0.244)$ & 0.178 & $(0.258)$ \\
\hline Neither & & & 0.159 & $(0.289)$ & 0.145 & $(0.338)$ & 0.145 & $(0.328)$ \\
\hline Weak Liberal & & & 0.215 & $(0.156)$ & 0.206 & $(0.176)$ & 0.207 & $(0.166)$ \\
\hline Liberal & & & $0.291^{+}$ & $(0.051)$ & $0.279^{+}$ & $(0.061)$ & $0.253^{+}$ & $(0.084)$ \\
\hline Strong Liberal & & & $0.466^{* *}$ & $(0.002)$ & $0.455^{* *}$ & $(0.003)$ & $0.424^{* *}$ & $(0.005)$ \\
\hline \multicolumn{9}{|l|}{ Age: } \\
\hline 31 to 40 & & & & & -0.0654 & $(0.137)$ & -0.0658 & $(0.145)$ \\
\hline 41 to 50 & & & & & -0.0636 & $(0.294)$ & -0.0786 & $(0.198)$ \\
\hline 51 to 60 & & & & & 0.00778 & $(0.911)$ & 0.00135 & $(0.985)$ \\
\hline$>60$ & & & & & -0.0874 & $(0.265)$ & -0.113 & $(0.165)$ \\
\hline \multicolumn{9}{|l|}{ Education: } \\
\hline High School & & & & & & & -0.0873 & $(0.591)$ \\
\hline Some College & & & & & & & -0.0301 & $(0.849)$ \\
\hline Bachelors & & & & & & & 0.0118 & $(0.941)$ \\
\hline Graduate & & & & & & & -0.0259 & $(0.872)$ \\
\hline \multicolumn{9}{|l|}{ Minority: } \\
\hline High Status & & & & & & & 0.0683 & $(0.140)$ \\
\hline Disadvantaged & & & & & & & $-0.117^{*}$ & $(0.012)$ \\
\hline \multicolumn{9}{|l|}{ Gender: } \\
\hline Female & & & & & & & $0.162^{* * *}$ & $(0.000)$ \\
\hline Other & & & & & & & $0.406^{* *}$ & $(0.008)$ \\
\hline Constant & $2.296^{* * *}$ & $(0.000)$ & $2.297^{* * *}$ & $(0.000)$ & $2.297^{* * *}$ & $(0.000)$ & $2.296^{* * *}$ & $(0.000)$ \\
\hline Observations & 10183 & & 10183 & & 10183 & & 10183 & \\
\hline Adjusted $R^{2}$ & 0.459 & & 0.461 & & 0.461 & & 0.464 & \\
\hline
\end{tabular}

p-values in parentheses. ${ }^{+} p<0.10,{ }^{*} p<0.05,{ }^{* *} p<0.01,{ }^{* * *} p<0.001$

All models include scenario and respondent fixed effects. Standard errors clustered by respondent. 


\section{Figures}

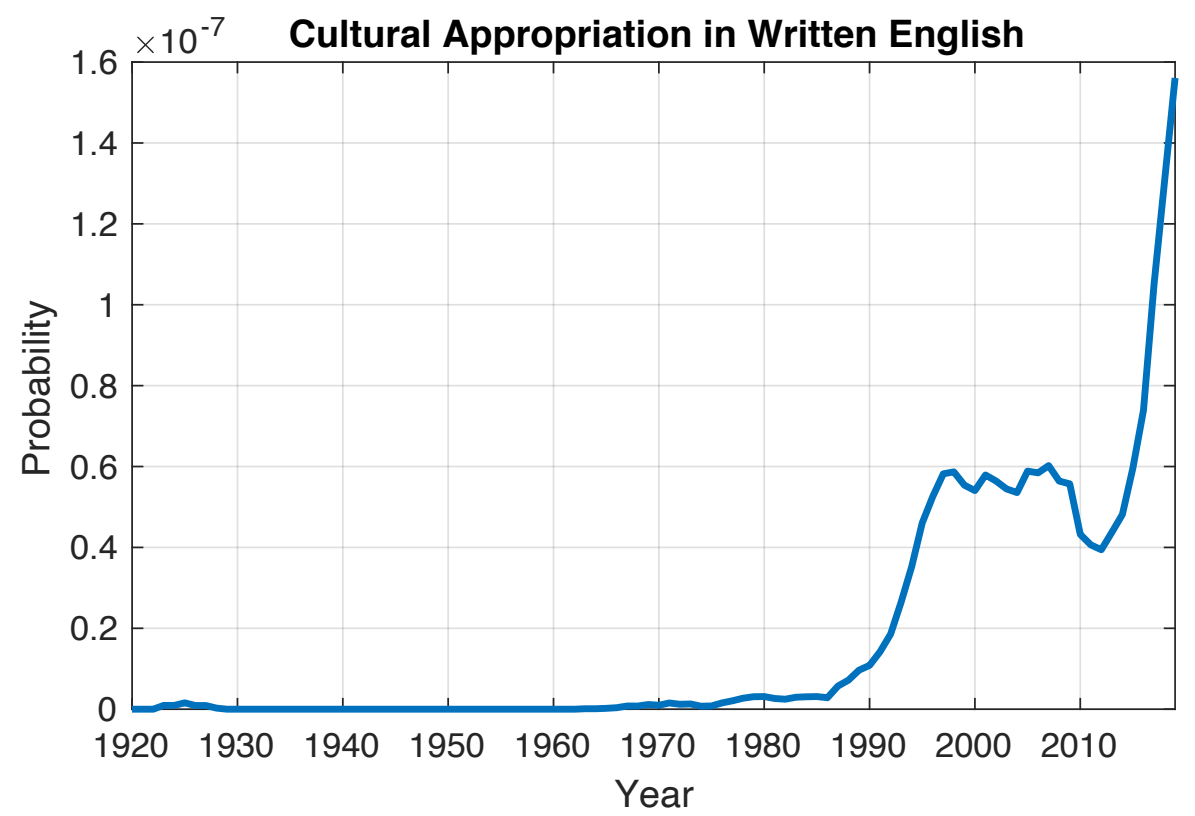

Figure 1: The probability, by year, of the bigram "Cultural Appropriation" as indexed by the Google Ngram Viewer project. The probability is calculated as the likelihood of the term appearing in a document during that year. The Google Ngram Viewer is the most comprehensive collection of texts published in the English language, containing upwards of 8 million books. 


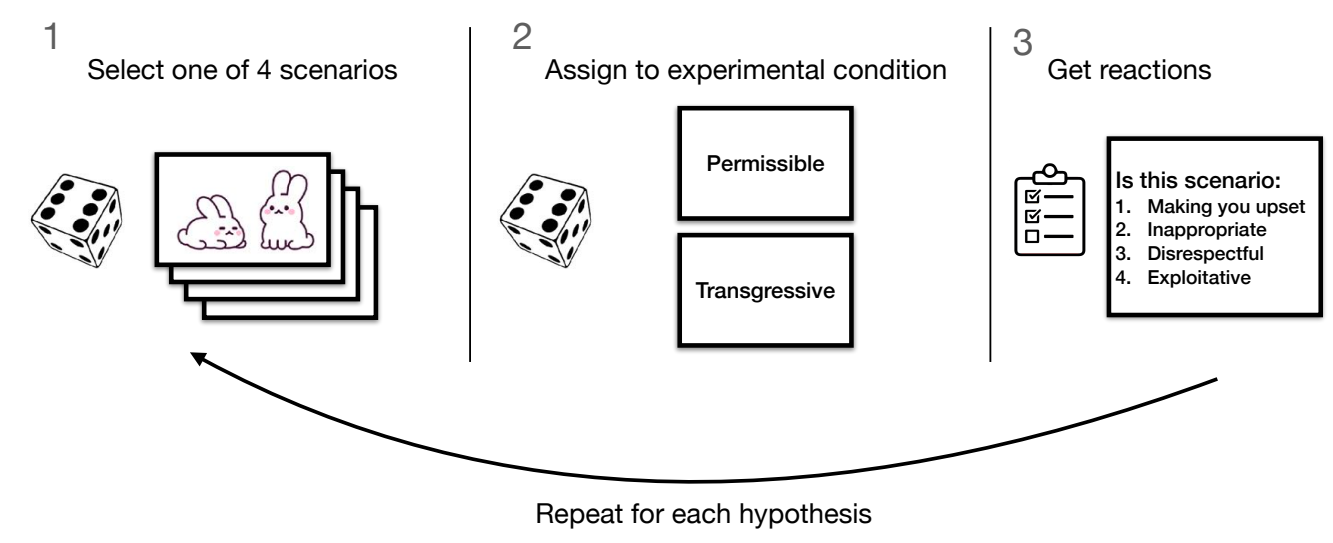

Figure 2: A schematic overview of the experimental procedure.

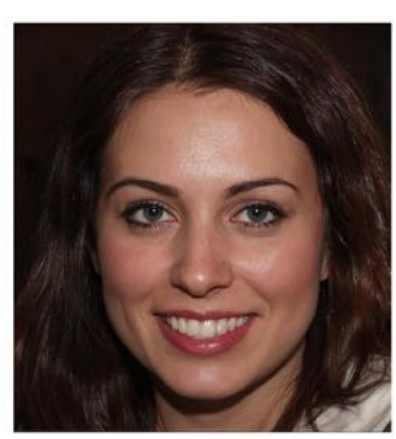

\section{Permissible}

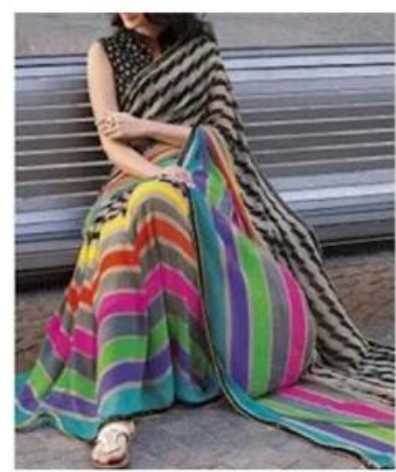

Lauren Anderson, 28, works as a cashier at a car wash facility in Los Angeles. She rents a modest apartment with her three housemates. Lauren has recently discovered Indian fashion, and has begun incorporating traditional Indian saris into her regular outfits. She especially likes to wear these at work, where she feels they make her "stand out."

\section{Transgressive}

Lauren Anderson, 28, works in her family's boutique real estate agency that caters to some of the wealthiest residents of Los Angeles. She lives in a beachfront house owned by her parents. Lauren has recently discovered Indian fashion, and has begun incorporating traditional Indian saris into her regular outfits. She especially likes to wear these at work, where she feels they make her "stand out."

Figure 3: Example of a scenario (2c) testing the intragroup hypothesis (H2). Subjects in both conditions are presented with the same two images and slightly modified accompanying texts. The differences between the texts presented in each condition are highlighted. 


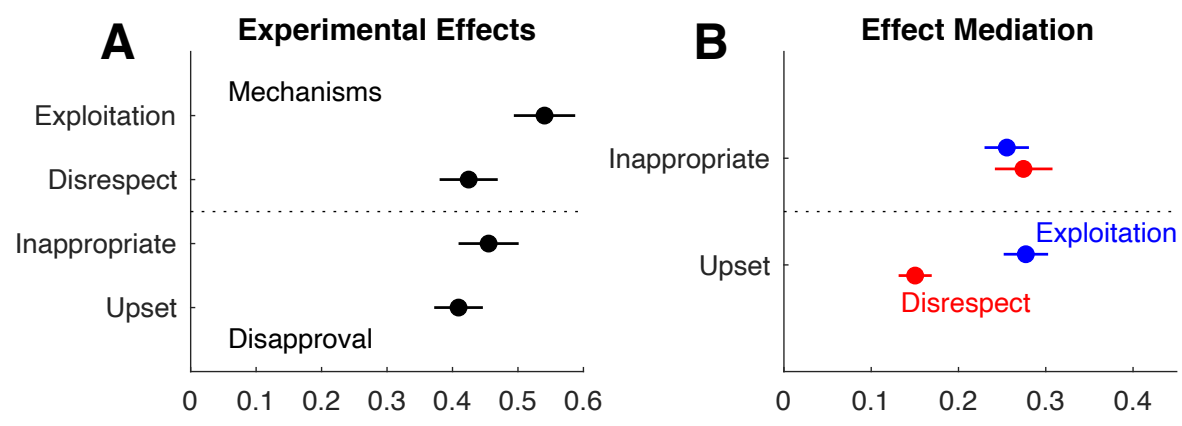

Figure 4: (A) The effect of being in the transgressive condition (relative to the permissible condition) on respondents' reactions. Coefficients were estimated using linear models, pooled across all scenarios, with respondent and scenario fixed effects (standard errors clustered by respondent). (B) Indirect effects estimated from a mediation model with disapproval (either upset or inappropriate) as the dependent variable and disrespect and exploitation as mediators (with scenario fixed effects). 

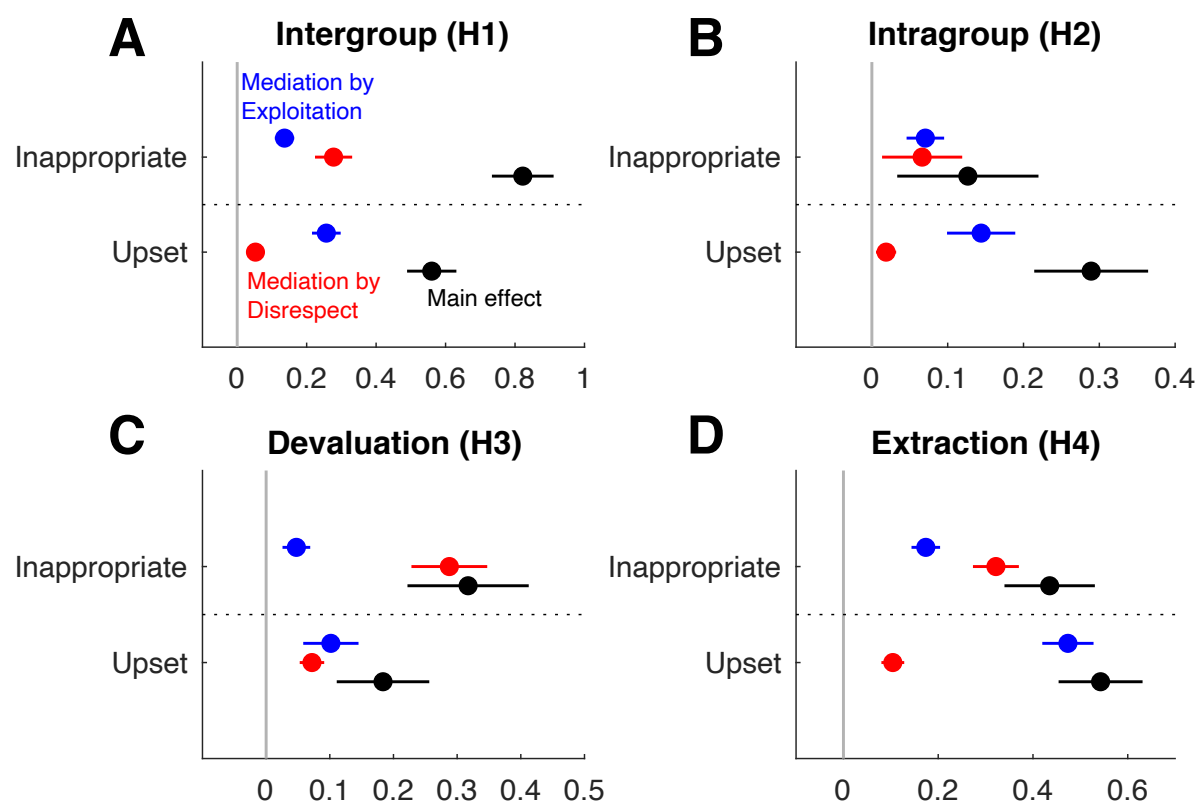

Figure 5: Experimental effects on disapproval by hypothesis. Each panel plots coefficients estimated by a linear model with scenario fixed effects (with $95 \%$ confidence intervals). Black dots correspond to the main effect of being in the transgressive condition. Blue and red dots correspond, respectively, to the indirect effect mediated by disrespect and exploitation. 

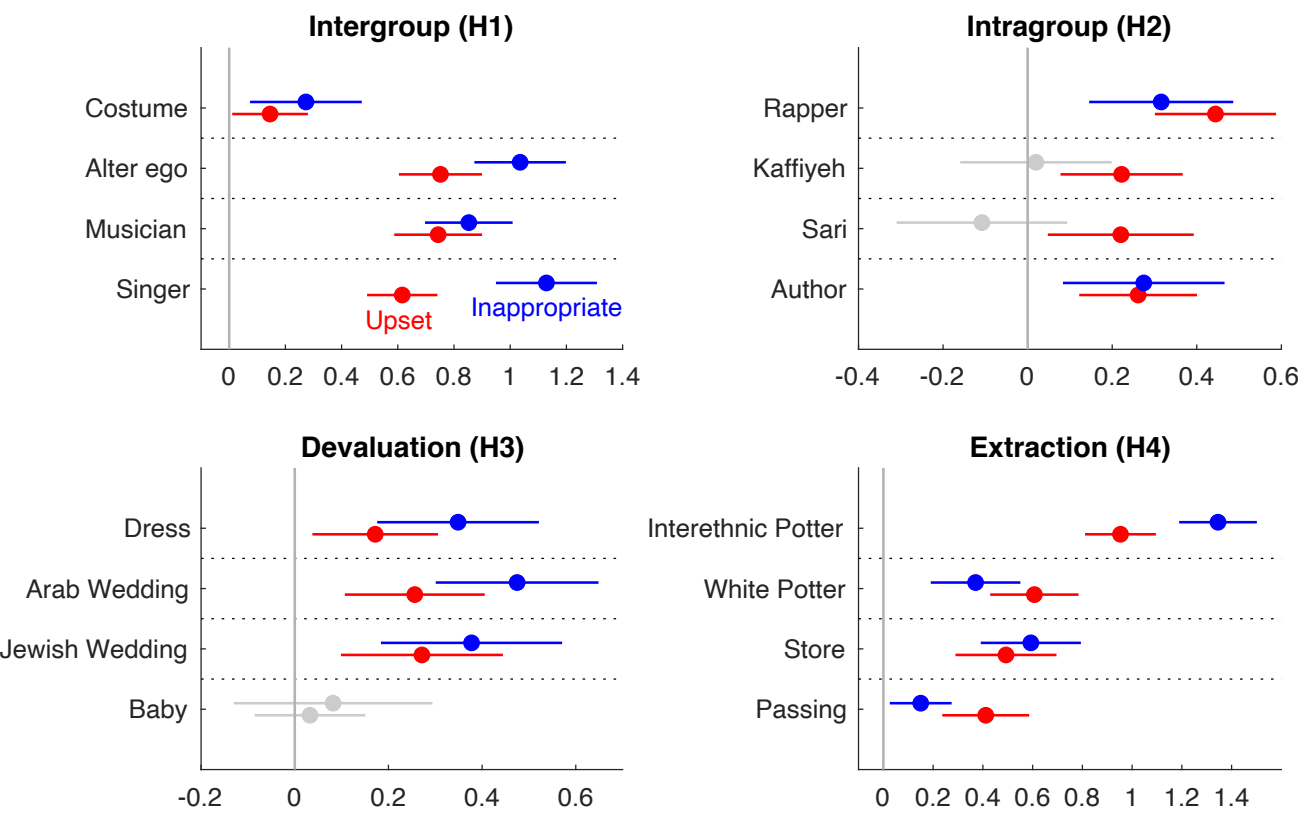

Figure 6: Disapproval by stimulus. The effect of being in the transgressive condition, relative to the permissible condition, on disapproval (upset in red and inappropriate in blue), by individual scenario (with 95\% confidence intervals). Grayed coefficients are insignificant. 



Figure 7: Moderation models. Each panel plots marginal effects (with 95\% confidence intervals) of an individual trait's moderation of the experimental effect on disapproval. Moderators are estimated independently. All models include scenario fixed effects. (A) Intergroup mobility disenchantment. (B) Intergroup mobility disenchantment (applied only to scenarios testing H1). (C) Intergroup mobility disenchantment (applied only to scenarios not testing H1). (D) Ideological identification. (E) Age. (F) Educational attainment. 

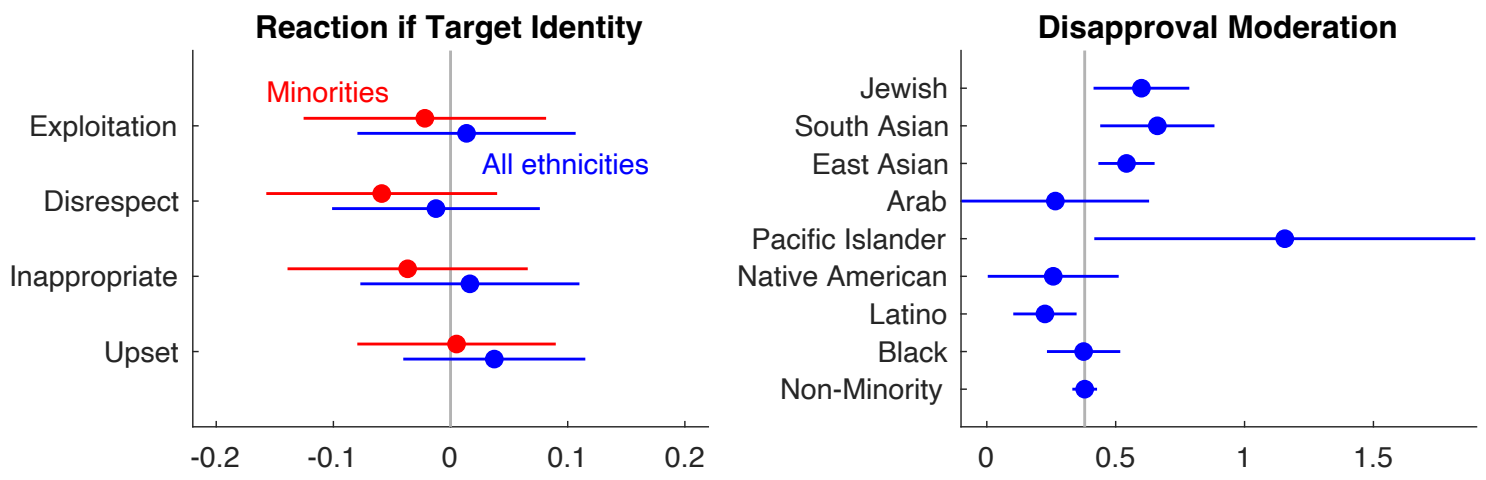

Figure 8: Sensitivity to cultural boundary-crossing by race/ethnicity. (A) The effect of having the target identity on reactions for all respondents (blue) or minority respondents only (red), as estimated by a model with respondent and scenario-condition fixed effects. (B) The moderation effect of being in the transgressive condition on disapproval, moderated by identity, estimated by a model with scenario fixed effects. The gray line corresponds to the main moderation effect of the omitted category (self-reported race is White or none). 


\section{A Variables and Descriptive Statistics}

\section{Experiment Variables}

Respondents were presented four vignettes, each depicting one scenario. After each vignette they were asked to provide their reactions to the following questions:

Upset: "Does [adopter's] behavior make you upset?"

Inappropriate: "Is [adopter's] behavior appropriate?" (reverse coded)

Disrespect: "Does [adopter] seem respectful of [target] culture to you?" (reverse coded)

Exploitation: "Do you think [adopter] is taking advantage of [target] culture?"

All reactions are scaled on a 5-point Likert scale which included the options "Not at all", "A little", "Moderately", "Quite a bit", and "Extremely". Reactions were randomly ordered for each respondent. Descriptive statistics and rank correlations are reported in Table A1.

For each vignette we altered the specific wordings of the questions in order to appropriately match the scenario contained within the vignette. For instance, for the Alter Ego stimulus, which features an adopter named Nick who light-heartedly dresses up as stereotypes of different cultural/ethnic groups, the question prompting upset is phrased as "Does Nick's behavior make you feel upset?". In the Passing scenario, where white adopter Mary ethnically "passes" as a Latina job applicant "Maria," we phrase the disrespect and exploitation questions as "Does Mary seem respectful of Hispanic culture to you?" and "Is Mary taking advantage of Hispanic culture?".

Competence: In addition to these reactions we also asked respondents about their perception of the adopter's competence for stimuli testing the extraction hypothesis (H4). These were worded as follows:

4a: "Does Mary seem like a capable worker?"

4b: "Does David seem like a smart businessman to you?"

4c and 4d: "Do you think [Daniel/Nolan] is a talented craftsman?"

The variable Disapproval is constructed by averaging upset and inappropriate. 
Table A1: Descriptive Statistics and Rank Correlations (Reactions)

\begin{tabular}{lcccccccc}
\hline \hline Variables & & & & & & & & \\
& $(1)$ & $(2)$ & $(3)$ & $\mathrm{N}$ & Min & Max & Mean & SD \\
\hline (1) Upset & 1.00 & & & 10,471 & 1 & 5 & 1.737 & 1.078 \\
(2) Inappropriate & 0.58 & 1.00 & & 10,266 & 1 & 5 & 3.286 & 1.329 \\
(3) Disrespect & 0.52 & 0.74 & 1.00 & 10,236 & 1 & 5 & 3.265 & 1.313 \\
(4) Exploitation & 0.67 & 0.58 & 0.54 & 10,354 & 1 & 5 & 2.259 & 1.321 \\
\hline \hline
\end{tabular}

\section{Respondent Demographics}

We collected a variety of demographic variables. Their descriptive statistics and correlations are presented in Table A2. These variables were collected and constructed as follows:

Age: Age in years.

Gender: Male, Female, Other (please specify).

Race: "What is your race?". Options include: White/Caucasian, Black/African descent, American Indian or Alaskan Native, Native Hawaiian or Pacific Islander, East Asian, South Asian, Other (please specify). Multiple selection permitted.

Latino: "Are you of Hispanic, Latino, or Spanish origin?" Yes/No.

Identity: "Do you self identify as" Arab or Middle-Eastern, Jewish. Multiple selection permitted.

Education: Some high-school or less, High-school, Some college or Associate's degree, Bachelor's degree, Graduate degree.

Ideology: 7-point Likert scale of ideological identification, including "Extremely Liberal", "Liberal", "Slightly Liberal", "Moderate or middle of the road", "Slightly Conservative", "Conservative", "Extremely Conservative", and "Something else".

Disenchantment: Respondent were asked how much they agree with the statement "It is less likely that an American youth who is a racial minority will go on to become a successful individual compared to the typical White youth, even if he or she is talented and hard-working." Agreement was measured using a 5-point Likert scale which includes the options "Strongly Disagree", "Disagree", "Neither agree nor disagree", "Agree", and "Strongly Agree".

The race, Latino and identity variables were transformed into dummy variables (given that options are not mutually exclusive). When respondents selected "Other" and specified a racial identity it was coded as one of those dummies if applicable (for example, "Mexican" 
was coded as Latino). Respondents identifying as Black, Native-American, Latino or Arab were coded as Disadvantaged Minority. All other minorities were coded as High-Status Minority. 
Table A2: Descriptive Statistics and Rank Correlations (Demographics)

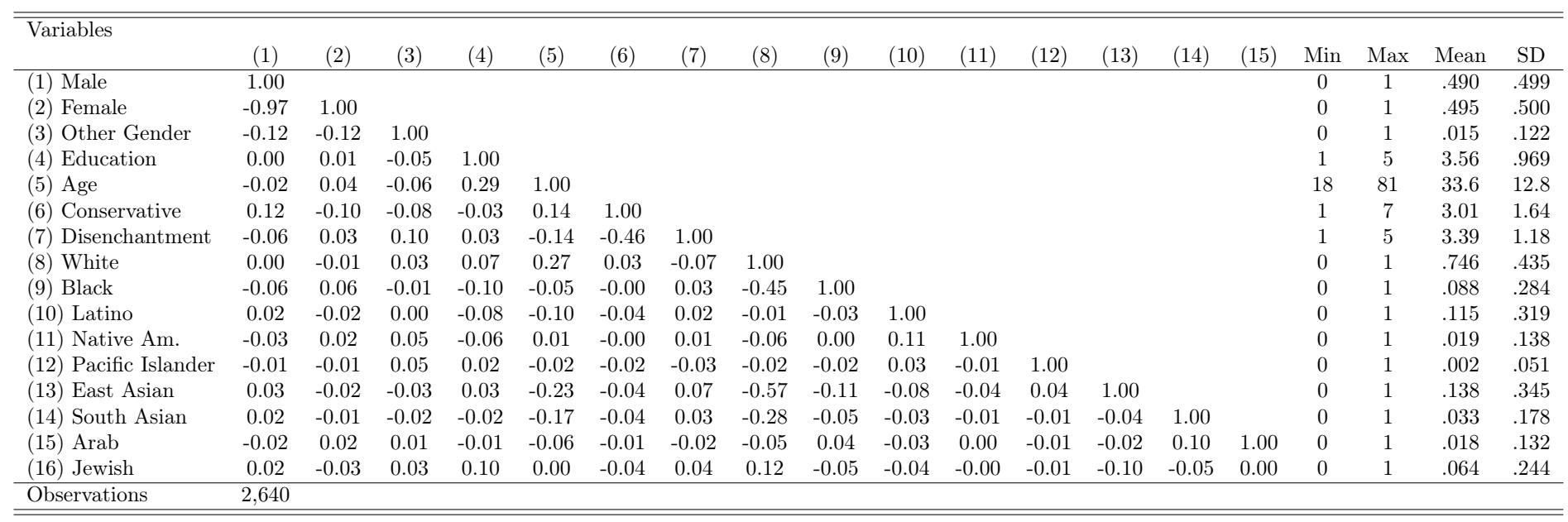




\section{B Analyses}

In this section we provide the full regression tables of results reported in graphical form in the main text, as well as additional results referred to in the main text but not presented in graphical form.

Table A3: Pooled Experimental Effect with Scenario and Respondent Fixed Effects

\begin{tabular}{lcccc}
\hline \hline & $(1)$ & $(2)$ & $(3)$ & $(4)$ \\
& Upset & Inappropriate & Disrespect & Exploitation \\
\hline Transgressive & $0.409^{* * *}$ & $0.455^{* * *}$ & $0.425^{* * *}$ & $0.541^{* * *}$ \\
& $(0.000)$ & $(0.000)$ & $(0.000)$ & $(0.000)$ \\
& & & & \\
Constant & $1.532^{* * *}$ & $3.054^{* * *}$ & $3.049^{* * *}$ & $1.989^{* * *}$ \\
& $(0.000)$ & $(0.000)$ & $(0.000)$ & $(0.000)$ \\
\hline Scenario FE & Yes & Yes & Yes & Yes \\
Respondent FE & Yes & Yes & Yes & Yes \\
Observations & 10471 & 10264 & 10231 & 10344 \\
$R^{2}$ & 0.581 & 0.578 & 0.581 & 0.559 \\
\hline \hline
\end{tabular}

$p$-values in parentheses, ${ }^{*} p<0.05,{ }^{* *} p<0.01,{ }^{* * *} p<0.001$

Standard errors clustered by respondent, Results reported in Figure 4 
Table A4: Experimental Effect by Hypothesis with Scenario Fixed Effects

\begin{tabular}{|c|c|c|c|c|c|c|c|c|}
\hline & \multicolumn{2}{|c|}{$\mathrm{H} 1$} & \multicolumn{2}{|c|}{$\mathrm{H} 2$} & \multicolumn{2}{|c|}{ H3 } & \multicolumn{2}{|c|}{$\mathrm{H} 4$} \\
\hline & (1) & $(2)$ & (3) & (4) & $(5)$ & (6) & $(7)$ & (8) \\
\hline & Upset & Inapp. & Upset & Inapp. & Upset & Inapp. & Upset & Inapp. \\
\hline Transgressive & $\begin{array}{c}0.560^{* * *} \\
(0.000)\end{array}$ & $\begin{array}{c}0.822^{* * *} \\
(0.000)\end{array}$ & $\begin{array}{c}0.289^{* * *} \\
(0.000)\end{array}$ & $\begin{array}{c}0.127^{* *} \\
(0.007)\end{array}$ & $\begin{array}{c}0.184^{* * *} \\
(0.000)\end{array}$ & $\begin{array}{c}0.317^{* * *} \\
(0.000)\end{array}$ & $\begin{array}{c}0.543^{* * *} \\
(0.000)\end{array}$ & $\begin{array}{c}0.435^{* * *} \\
(0.000)\end{array}$ \\
\hline Constant & $\begin{array}{c}1.318^{* * *} \\
(0.000)\end{array}$ & $\begin{array}{c}2.813^{* * *} \\
(0.000)\end{array}$ & $\begin{array}{c}1.487^{* * *} \\
(0.000)\end{array}$ & $\begin{array}{c}3.155^{* * *} \\
(0.000)\end{array}$ & $\begin{array}{c}1.530^{* * *} \\
(0.000)\end{array}$ & $\begin{array}{c}3.153^{* * *} \\
(0.000)\end{array}$ & $\begin{array}{c}1.823^{* * *} \\
(0.000)\end{array}$ & $\begin{array}{c}3.159^{* * *} \\
(0.000)\end{array}$ \\
\hline Scenario FE & Yes & Yes & Yes & Yes & Yes & Yes & Yes & Yes \\
\hline Observations & 2593 & 2593 & 2633 & 2564 & 2613 & 2496 & 2632 & 2613 \\
\hline$R^{2}$ & 0.111 & 0.230 & 0.0349 & 0.0610 & 0.0450 & 0.127 & 0.154 & 0.266 \\
\hline
\end{tabular}

$p$-values in parentheses, ${ }^{*} p<0.05,{ }^{* *} p<0.01,{ }^{* * *} p<0.001$, Standard errors clustered by respondent

Results reported in Figure 5 
Table A5: Experimental Effect on Disapproval, by Scenario

\begin{tabular}{|c|c|c|c|c|c|c|c|c|}
\hline & \multicolumn{8}{|c|}{ Intergroup Hypothesis (H1) } \\
\hline & \multicolumn{2}{|c|}{ Singer } & \multicolumn{2}{|c|}{ Musician } & \multicolumn{2}{|c|}{ Alter Ego } & \multicolumn{2}{|c|}{ Costume } \\
\hline & Upset & Inapp. & Upset & Inapp. & Upset & Inapp. & Upset & Inapp. \\
\hline \multirow[t]{2}{*}{ Transgressive } & $0.616^{* * *}$ & $1.129^{* * *}$ & $0.744^{* * *}$ & $0.853^{* * *}$ & $0.752^{* * *}$ & $1.035^{* * *}$ & $0.145^{*}$ & $0.273^{* *}$ \\
\hline & $(0.000)$ & $(0.000)$ & $(0.000)$ & $(0.000)$ & $(0.000)$ & $(0.000)$ & $(0.034)$ & $(0.007)$ \\
\hline \multirow[t]{2}{*}{ Constant } & $1.139^{* * *}$ & $2.161^{* * *}$ & $1.469^{* * *}$ & $3.349^{* * *}$ & $1.320^{* * *}$ & $3.121^{* * *}$ & $1.361^{* * *}$ & $2.673^{* * *}$ \\
\hline & $(0.000)$ & $(0.000)$ & $(0.000)$ & $(0.000)$ & $(0.000)$ & $(0.000)$ & $(0.000)$ & $(0.000)$ \\
\hline Observations & 665 & 665 & 602 & 602 & 664 & 664 & 662 & 662 \\
\hline \multirow[t]{4}{*}{$R^{2}$} & 0.123 & 0.187 & 0.127 & 0.161 & 0.131 & 0.191 & 0.007 & 0.011 \\
\hline & \multicolumn{8}{|c|}{ Intragroup Hypothesis (H2) } \\
\hline & \multicolumn{2}{|c|}{ Author } & \multicolumn{2}{|c|}{ Sari } & \multicolumn{2}{|c|}{ Kaffiyeh } & \multicolumn{2}{|c|}{ Rapper } \\
\hline & Upset & Inapp. & Upset & Inapp. & Upset & Inapp. & Upset & Inapp. \\
\hline \multirow{2}{*}{ Transgressive } & $0.261^{* * *}$ & $0.275^{* *}$ & $0.220^{*}$ & -0.108 & $0.222^{* *}$ & 0.0196 & $0.444^{* * *}$ & $0.316^{* * *}$ \\
\hline & $(0.000)$ & $(0.005)$ & $(0.012)$ & $(0.293)$ & $(0.003)$ & $(0.830)$ & $(0.000)$ & $(0.000)$ \\
\hline \multirow[t]{2}{*}{ Constant } & $1.363^{* * *}$ & $2.584^{* * *}$ & $1.714^{* * *}$ & $3.413^{* * *}$ & $1.497^{* * *}$ & $3.500^{* * *}$ & $1.378^{* * *}$ & $3.132^{* * *}$ \\
\hline & $(0.000)$ & $(0.000)$ & $(0.000)$ & $(0.000)$ & $(0.000)$ & $(0.000)$ & $(0.000)$ & $(0.000)$ \\
\hline Observations & 647 & 647 & 650 & 650 & 649 & 617 & 687 & 650 \\
\hline \multirow[t]{4}{*}{$R^{2}$} & 0.021 & 0.012 & 0.010 & 0.002 & 0.014 & 0.000 & 0.051 & 0.020 \\
\hline & \multicolumn{8}{|c|}{ Devaluation Hypothesis (H3) } \\
\hline & \multicolumn{2}{|c|}{ Baby } & \multicolumn{2}{|c|}{ Jewish Wedding } & \multicolumn{2}{|c|}{ Arab Wedding } & \multicolumn{2}{|c|}{ Dress } \\
\hline & Upset & Inapp. & Upset & Inapp. & Upset & Inapp. & Upset & Inapp. \\
\hline \multirow[t]{2}{*}{ Transgressive } & 0.0325 & 0.0818 & $0.272^{* *}$ & $0.377^{* * *}$ & $0.256^{* * *}$ & $0.475^{* * *}$ & $0.172^{*}$ & $0.349^{* * *}$ \\
\hline & $(0.589)$ & $(0.449)$ & $(0.002)$ & $(0.000)$ & $(0.001)$ & $(0.000)$ & $(0.012)$ & $(0.000)$ \\
\hline \multirow[t]{2}{*}{ Constant } & $1.341^{* * *}$ & $2.563^{* * *}$ & $1.713^{* * *}$ & $3.439^{* * *}$ & $1.589^{* * *}$ & $3.420^{* * *}$ & $1.472^{* * *}$ & $3.204^{* * *}$ \\
\hline & $(0.000)$ & $(0.000)$ & $(0.000)$ & $(0.000)$ & $(0.000)$ & $(0.000)$ & $(0.000)$ & $(0.000)$ \\
\hline Observations & 650 & 650 & 667 & 667 & 649 & 602 & 647 & 577 \\
\hline \multirow[t]{4}{*}{$R^{2}$} & 0.000 & 0.001 & 0.014 & 0.022 & 0.017 & 0.046 & 0.010 & 0.027 \\
\hline & \multicolumn{8}{|c|}{ Extraction Hypothesis (H4) } \\
\hline & \multicolumn{2}{|c|}{ Passing } & & & White & Potter & Intereth & c Potter \\
\hline & Upset & Inapp. & Upset & Inapp. & Upset & Inapp. & Upset & Inapp. \\
\hline Transgressive & $0.411^{* * *}$ & $0.150^{*}$ & $0.493^{* * *}$ & $0.593^{* * *}$ & $0.607^{* * *}$ & $0.371^{* * *}$ & $0.953^{* * *}$ & $1.345^{* * *}$ \\
\hline & $(0.000)$ & $(0.018)$ & $(0.000)$ & $(0.000)$ & $(0.000)$ & $(0.000)$ & $(0.000)$ & $(0.000)$ \\
\hline Competence & Yes & Yes & Yes & Yes & Yes & Yes & Yes & Yes \\
\hline Constant & $3.396^{* * *}$ & $4.837^{* * *}$ & $2.026^{* * *}$ & $4.312^{* * *}$ & $3.117^{* * *}$ & $4.360^{* * *}$ & $1.741^{* * *}$ & $3.231^{* * *}$ \\
\hline & $(0.000)$ & $(0.000)$ & $(0.000)$ & $(0.000)$ & $(0.000)$ & $(0.000)$ & $(0.000)$ & $(0.000)$ \\
\hline Observations & 686 & 686 & 627 & 610 & 650 & 650 & 660 & 660 \\
\hline$R^{2}$ & 0.234 & 0.395 & 0.096 & 0.347 & 0.157 & 0.253 & 0.338 & 0.521 \\
\hline
\end{tabular}

$p$-values in parentheses, ${ }^{*} p<0.05,{ }^{* *} p<0.01,{ }^{* * *} p<0.001$, Results reported in Figure 6 
Table A6: Experimental Effect on Mediators, by Scenario

\begin{tabular}{|c|c|c|c|c|c|c|c|c|}
\hline & \multicolumn{8}{|c|}{ Intergroup Hypothesis (H1) } \\
\hline & \multicolumn{2}{|c|}{ Singer } & \multicolumn{2}{|c|}{ Musician } & \multicolumn{2}{|c|}{ Alter Ego } & \multicolumn{2}{|c|}{ Costume } \\
\hline & Dis. & Exp. & Dis. & Exp. & Dis. & Exp. & Dis. & Exp. \\
\hline \multirow[t]{2}{*}{ Transgressive } & $0.926^{* * *}$ & $0.954^{* * *}$ & $0.451^{* * *}$ & $0.371^{* * *}$ & $0.639^{* * *}$ & $1.091^{* * *}$ & 0.101 & 0.0675 \\
\hline & $(0.000)$ & $(0.000)$ & $(0.000)$ & $(0.000)$ & $(0.000)$ & $(0.000)$ & $(0.313)$ & $(0.445)$ \\
\hline \multirow[t]{2}{*}{ Constant } & $2.327^{* * *}$ & $1.488^{* * *}$ & $3.593^{* * *}$ & $2.329^{* * *}$ & $3.553^{* * *}$ & $1.903^{* * *}$ & $2.673^{* * *}$ & $1.791^{* * *}$ \\
\hline & $(0.000)$ & $(0.000)$ & $(0.000)$ & $(0.000)$ & $(0.000)$ & $(0.000)$ & $(0.000)$ & $(0.000)$ \\
\hline Observations & 665 & 665 & 611 & 552 & 664 & 664 & 662 & 662 \\
\hline \multirow[t]{4}{*}{$R^{2}$} & 0.134 & 0.153 & 0.046 & 0.027 & 0.074 & 0.165 & 0.002 & 0.001 \\
\hline & \multicolumn{8}{|c|}{ Intragroup Hypothesis (H2) } \\
\hline & \multicolumn{2}{|c|}{ Author } & \multicolumn{2}{|c|}{ Sari } & \multicolumn{2}{|c|}{ Kaffiyeh } & \multicolumn{2}{|c|}{ Rapper } \\
\hline & Dis. & Exp. & Dis. & Exp. & Dis. & Exp. & Dis. & Exp. \\
\hline \multirow[t]{2}{*}{ Transgressive } & $0.270^{* *}$ & $0.330^{* * *}$ & -0.101 & 0.120 & 0.0567 & $0.331^{* * *}$ & $0.261^{* *}$ & $0.566^{* * *}$ \\
\hline & $(0.004)$ & $(0.000)$ & $(0.331)$ & $(0.262)$ & $(0.520)$ & $(0.000)$ & $(0.002)$ & $(0.000)$ \\
\hline \multirow{2}{*}{ Constant } & $2.469^{* * *}$ & $1.863^{* * *}$ & $3.453^{* * *}$ & $2.447^{* * *}$ & $3.605^{* * *}$ & $1.971^{* * *}$ & $3.348^{* * *}$ & $1.839^{* * *}$ \\
\hline & $(0.000)$ & $(0.000)$ & $(0.000)$ & $(0.000)$ & $(0.000)$ & $(0.000)$ & $(0.000)$ & $(0.000)$ \\
\hline Observations & 647 & 647 & 650 & 650 & 617 & 641 & 667 & 684 \\
\hline \multirow[t]{4}{*}{$R^{2}$} & 0.013 & 0.021 & 0.001 & 0.002 & 0.001 & 0.020 & 0.014 & 0.055 \\
\hline & \multicolumn{8}{|c|}{ Devaluation Hypothesis (H3) } \\
\hline & \multicolumn{2}{|c|}{ Baby } & \multicolumn{2}{|c|}{ Jewish Wedding } & \multicolumn{2}{|c|}{ Arab Wedding } & \multicolumn{2}{|c|}{ Dress } \\
\hline & Dis. & Exp. & Dis. & Exp. & Dis. & Exp. & Dis. & Exp. \\
\hline \multirow[t]{2}{*}{ Transgressive } & $0.515^{* * *}$ & 0.00236 & $0.446^{* * *}$ & $0.250^{*}$ & $0.676^{* * *}$ & $0.416^{* * *}$ & $0.372^{* * *}$ & $0.240^{* *}$ \\
\hline & $(0.000)$ & $(0.975)$ & $(0.000)$ & $(0.018)$ & $(0.000)$ & $(0.000)$ & $(0.000)$ & $(0.002)$ \\
\hline \multirow[t]{2}{*}{ Constant } & $2.663^{* * *}$ & $1.557^{* * *}$ & $3.039^{* * *}$ & $2.361^{* * *}$ & $3.037^{* * *}$ & $1.833^{* * *}$ & $3.084^{* * *}$ & $1.722^{* * *}$ \\
\hline & $(0.000)$ & $(0.000)$ & $(0.000)$ & $(0.000)$ & $(0.000)$ & $(0.000)$ & $(0.000)$ & $(0.000)$ \\
\hline$\overline{\text { Observations }}$ & 650 & 650 & 667 & 667 & 579 & 605 & 557 & 632 \\
\hline \multirow[t]{4}{*}{$R^{2}$} & 0.039 & 0.000 & 0.029 & 0.008 & 0.092 & 0.035 & 0.032 & 0.014 \\
\hline & \multicolumn{8}{|c|}{ Extraction Hypothesis (H4) } \\
\hline & \multicolumn{2}{|c|}{ Passing } & & & White & Potter & Intereth & c Potter \\
\hline & Dis. & Exp. & Dis. & Exp. & Dis. & Exp. & Dis. & Exp. \\
\hline Transgressive & 0.0789 & $0.433^{* * *}$ & $0.526^{* * *}$ & $0.725^{* * *}$ & $0.530^{* * *}$ & $1.116^{* * *}$ & $1.199^{* * *}$ & $1.389^{* * *}$ \\
\hline & $(0.234)$ & $(0.000)$ & $(0.000)$ & $(0.000)$ & $(0.000)$ & $(0.000)$ & $(0.000)$ & $(0.000)$ \\
\hline Competence & Yes & Yes & Yes & Yes & Yes & Yes & Yes & Yes \\
\hline Constant & $4.844^{* * *}$ & $4.197^{* * *}$ & $4.205^{* * *}$ & $2.026^{* * *}$ & $4.486^{* * *}$ & $3.213^{* * *}$ & $3.315^{* * *}$ & $2.063^{* * *}$ \\
\hline & $(0.000)$ & $(0.000)$ & $(0.000)$ & $(0.000)$ & $(0.000)$ & $(0.000)$ & $(0.000)$ & $(0.000)$ \\
\hline Observations & 686 & 686 & 601 & 621 & 650 & 650 & 660 & 660 \\
\hline$R^{2}$ & 0.390 & 0.259 & 0.314 & 0.092 & 0.297 & 0.208 & 0.511 & 0.341 \\
\hline
\end{tabular}


Table A7: Moderation of Experimental Effect on Disapproval by Mobility Disenchantment

\begin{tabular}{|c|c|c|c|c|c|c|}
\hline & $\begin{array}{c}(1) \\
\text { All stimuli }\end{array}$ & & $\begin{array}{c}(2) \\
\text { H1 Only }\end{array}$ & & $\begin{array}{c}(3) \\
\text { Not H1 }\end{array}$ & \\
\hline Transgressive & $0.209^{* *}$ & $(0.004)$ & $0.481^{* * *}$ & $(0.000)$ & 0.115 & $(0.176)$ \\
\hline \multicolumn{7}{|l|}{ Disenchantment } \\
\hline Disagree & $0.135^{*}$ & $(0.037)$ & $0.187^{*}$ & $(0.042)$ & 0.113 & $(0.125)$ \\
\hline Neither & $0.238^{* * *}$ & $(0.000)$ & $0.268^{* *}$ & $(0.003)$ & $0.226^{* *}$ & $(0.003)$ \\
\hline Agree & $0.373^{* * *}$ & $(0.000)$ & $0.357^{* * *}$ & $(0.000)$ & $0.372^{* * *}$ & $(0.000)$ \\
\hline Strongly Agree & $0.460^{* * *}$ & $(0.000)$ & $0.253^{*}$ & $(0.012)$ & $0.524^{* * *}$ & $(0.000)$ \\
\hline \multicolumn{7}{|l|}{ Disenchantment $\times$ Transgressive } \\
\hline Disagree $\times$ Transgressive & -0.000270 & $(0.997)$ & -0.0467 & $(0.742)$ & 0.0204 & $(0.836)$ \\
\hline Neither $\times$ Transgressive & 0.131 & $(0.120)$ & 0.0639 & $(0.657)$ & 0.157 & $(0.114)$ \\
\hline Agree $\times$ Transgressive & $0.242^{* *}$ & $(0.002)$ & 0.217 & $(0.095)$ & $0.255^{* *}$ & $(0.005)$ \\
\hline Strongly Agree $\times$ Transgressive & $0.467^{* * *}$ & $(0.000)$ & $0.632^{* * *}$ & $(0.000)$ & $0.416^{* * *}$ & $(0.000)$ \\
\hline Constant & $2.013^{* * *}$ & $(0.000)$ & $1.797^{* * *}$ & $(0.000)$ & $2.091^{* * *}$ & $(0.000)$ \\
\hline Stimulus FE & Yes & & Yes & & Yes & \\
\hline Observations & 10185 & & 2551 & & 7634 & \\
\hline$R^{2}$ & 0.218 & & 0.256 & & 0.212 & \\
\hline
\end{tabular}

$p$-values in parentheses, ${ }^{*} p<0.05,{ }^{* *} p<0.01,{ }^{* * *} p<0.001$, Standard errors clustered by respondent

Results reported in Figure 7A-C 
Table A8: Moderation of Experimental Effect on Disapproval by Ideology, Age and Education

\begin{tabular}{|c|c|c|c|c|c|c|}
\hline & \multicolumn{2}{|c|}{ 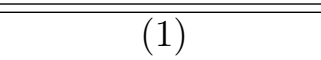 } & \multicolumn{2}{|c|}{ 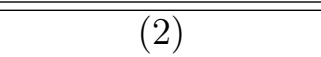 } & \multicolumn{2}{|c|}{ 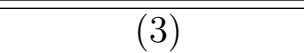 } \\
\hline Transgressive & $0.669^{* * *}$ & $(0.000)$ & $0.481^{* * *}$ & $(0.000)$ & $0.533^{* * *}$ & $(0.001)$ \\
\hline \multicolumn{7}{|l|}{ Political Ideology } \\
\hline Liberal & $-0.118^{* *}$ & $(0.009)$ & & & & \\
\hline Weak Liberal & $-0.189^{* * *}$ & $(0.000)$ & & & & \\
\hline Neither & $-0.251^{* * *}$ & $(0.000)$ & & & & \\
\hline Weak Conservative & $-0.343^{* * *}$ & $(0.000)$ & & & & \\
\hline Conservative & $-0.341^{* * *}$ & $(0.000)$ & & & & \\
\hline Strong Conservative & $-0.286^{*}$ & $(0.017)$ & & & & \\
\hline Liberal $\times$ Transgressive & $-0.203^{* * *}$ & $(0.000)$ & & & & \\
\hline Weak Liberal $\times$ Transgressive & $-0.296^{* * *}$ & $(0.000)$ & & & & \\
\hline Neither $\times$ Transgressive & $-0.392^{* * *}$ & $(0.000)$ & & & & \\
\hline Weak Conservative $\times$ Transgressive & $-0.448^{* * *}$ & $(0.000)$ & & & & \\
\hline Conservative $\times$ Transgressive & $-0.426^{* * *}$ & $(0.000)$ & & & & \\
\hline Strong Conservative $\times$ Transgressive & $-0.624^{* * *}$ & $(0.000)$ & & & & \\
\hline \multicolumn{7}{|l|}{ Age } \\
\hline 31 to 40 & & & -0.0573 & $(0.117)$ & & \\
\hline 41 to 50 & & & $-0.129^{* *}$ & $(0.007)$ & & \\
\hline 51 to 60 & & & $-0.145^{* *}$ & $(0.007)$ & & \\
\hline Above 60 & & & $-0.209^{* * *}$ & $(0.000)$ & & \\
\hline 31 to $40 \times$ Transgressive & & & $-0.127^{* *}$ & $(0.008)$ & & \\
\hline 41 to $50 \times$ Transgressive & & & $-0.195^{* *}$ & $(0.002)$ & & \\
\hline 51 to $60 \times$ Transgressive & & & $-0.158^{*}$ & $(0.030)$ & & \\
\hline Above $60 \times$ Transgressive & & & $-0.215^{* *}$ & $(0.004)$ & & \\
\hline \multicolumn{7}{|l|}{ Education } \\
\hline High School & & & & & 0.0268 & $(0.863)$ \\
\hline Some College & & & & & 0.0636 & $(0.677)$ \\
\hline Bachelors & & & & & 0.0776 & $(0.610)$ \\
\hline Graduate & & & & & 0.0699 & $(0.651)$ \\
\hline High School $\times$ Transgressive & & & & & -0.162 & $(0.330)$ \\
\hline Some College $\times$ Transgressive & & & & & -0.123 & $(0.445)$ \\
\hline Bachelors $\times$ Transgressive & & & & & -0.0907 & $(0.571)$ \\
\hline Graduate $\times$ Transgressive & & & & & -0.198 & $(0.225)$ \\
\hline Constant & $2.478^{* * *}$ & $(0.000)$ & $2.359^{* * *}$ & $(0.000)$ & $2.245^{* * *}$ & $(0.000)$ \\
\hline Stimulus FE & Yes & & Yes & & Yes & \\
\hline Observations & 10185 & & 10185 & & 10185 & \\
\hline$R^{2}$ & 0.212 & & 0.184 & & 0.175 & \\
\hline
\end{tabular}

$p$-values in parentheses, ${ }^{*} p<0.05,{ }^{* *} p<0.01,{ }^{* * *} p<0.001$, Standard errors clustered by respondent Results reported in Figure 7D-F 
Table A9: Effect of Having the Target Identity on Disapproval

\begin{tabular}{lcccccccc}
\hline \hline & $(1)$ & $(2)$ & $(3)$ & $(4)$ & $(5)$ & $(6)$ & $(7)$ & $(8)$ \\
& Upset & Inapp. & Dis. & Exp. & Upset & Inapp. & Dis. & Exp. \\
\hline Target & 0.0373 & 0.0165 & -0.0124 & 0.0136 & & & & \\
& $(0.346)$ & $(0.729)$ & $(0.783)$ & $(0.774)$ & & & & \\
Minority Target & & & & & & & & \\
& & & & & 0.00512 & -0.0366 & -0.0587 & -0.0219 \\
& & & & & $(0.906)$ & $(0.484)$ & $(0.242)$ & $(0.678)$ \\
Constant & $1.732^{* * *}$ & $3.284^{* * *}$ & $3.267^{* * *}$ & $2.257^{* * *}$ & $1.737^{* * *}$ & $3.289^{* * *}$ & $3.269^{* * *}$ & $2.261^{* * *}$ \\
& $(0.000)$ & $(0.000)$ & $(0.000)$ & $(0.000)$ & $(0.000)$ & $(0.000)$ & $(0.000)$ & $(0.000)$ \\
\hline Scen.-Condition FE & Yes & Yes & Yes & Yes & Yes & Yes & Yes & Yes \\
Respondent FE & Yes & Yes & Yes & Yes & Yes & Yes & Yes & Yes \\
Observations & 10471 & 10264 & 10231 & 10344 & 10471 & 10264 & 10231 & 10344 \\
$R^{2}$ & 0.593 & 0.601 & 0.597 & 0.576 & 0.593 & 0.601 & 0.597 & 0.576 \\
\hline \hline
\end{tabular}

$p$-values in parentheses, ${ }^{*} p<0.05,{ }^{* *} p<0.01,{ }^{* * *} p<0.001$, Standard errors clustered by respondent

Results reported in Figure 8A 
Table A10: Moderation of Experimental Effect on Disapproval by Racial/Ethnic Identity

\begin{tabular}{lcc}
\hline \hline & $(1)$ & \\
& Offense & $(0.000)$ \\
\hline Transgressive & $0.380^{* * *}$ & $(0.700)$ \\
Black & 0.0202 & $(0.954)$ \\
Transgressive $\times$ Black & -0.00422 & $(0.026)$ \\
Latino & $0.108^{*}$ & $(0.014)$ \\
Transgressive $\times$ Latino & $-0.154^{*}$ & $(0.177)$ \\
Native-American & 0.155 & $(0.346)$ \\
Transgressive $\times$ Native-American & -0.122 & $(0.069)$ \\
East Asian & 0.0745 & $(0.004)$ \\
Transgressive $\times$ East Asian & $0.162^{* *}$ & $(0.239)$ \\
South Asian & 0.0952 & $(0.013)$ \\
Transgressive $\times$ South Asian & $0.282^{*}$ & $(0.470)$ \\
Pacific Islander & -0.148 & $(0.040)$ \\
Transgressive $\times$ Pacific Islander & $0.776^{*}$ & $(0.777)$ \\
Jewish & 0.0196 & $(0.020)$ \\
Transgressive $\times$ Jewish & $0.220^{*}$ & $(0.455)$ \\
Arab & 0.0892 & $(0.539)$ \\
Transgressive $\times$ Arab & -0.114 & $(0.000)$ \\
Constant & $2.276^{* * *}$ & \\
\hline Scenario FE & Yes & \\
Observations & 10185 & \\
$R^{2}$ & 0.181 & \\
\hline \hline$p$-values in parentheses ${ }^{*} p<0.05,{ }^{* *} p<0.01,{ }^{* * *} p<0.001$, Standard errors clustered by respondent \\
Results reported in Figure $8 \mathrm{~B}$ & & \\
& & \\
\hline
\end{tabular}




\section{Competence}

In this section we provide further justification for our decision to include the adopter's perceived competence as a control in models testing the extraction hypothesis (H4). As Tables A11 and A12 demonstrate, the inclusion of this control in the model only affects results for stimulus 4b ("Store"). Once the perceived business competence of the store owner is accounted for, experimental effects are in the expected direction. Other stimuli are not affected.

Table A11: Experimental Effect on Upset by Scenario, Extraction Hypothesis (H4)

\begin{tabular}{|c|c|c|c|c|c|c|c|c|}
\hline & \multicolumn{2}{|c|}{ Passing } & \multicolumn{2}{|c|}{ Store } & \multicolumn{2}{|c|}{ "White Potter } & \multicolumn{2}{|c|}{ Interethnic Potter } \\
\hline & (1) & $(2)$ & $(3)$ & (4) & $(5)$ & $(6)$ & $(7)$ & $(8)$ \\
\hline Transgressive & $\begin{array}{c}0.503^{* * *} \\
(0.000)\end{array}$ & $\begin{array}{c}0.411^{\text {*** }} \\
(0.000)\end{array}$ & $\begin{array}{c}0.00483 \\
(0.955)\end{array}$ & $\begin{array}{c}0.493^{* * *} \\
(0.000)\end{array}$ & $\begin{array}{c}0.529^{* * *} \\
(0.000)\end{array}$ & $\begin{array}{c}0.607^{* * *} \\
(0.000)\end{array}$ & $\begin{array}{c}1.116^{* * *} \\
(0.000)\end{array}$ & $\begin{array}{c}0.953^{* * *} \\
(0.000)\end{array}$ \\
\hline Competent & & & & & & & & \\
\hline A little & & $\begin{array}{c}-0.669^{* * *} \\
(0.000)\end{array}$ & & $\begin{array}{c}-0.0988 \\
(0.430)\end{array}$ & & $\begin{array}{l}-0.821^{*} \\
(0.013)\end{array}$ & & $\begin{array}{c}0.354 \\
(0.340)\end{array}$ \\
\hline Moderately & & $\begin{array}{c}-1.015^{* * *} \\
(0.000)\end{array}$ & & $\begin{array}{c}-0.373^{* *} \\
(0.004)\end{array}$ & & $\begin{array}{c}-0.899^{* *} \\
(0.004)\end{array}$ & & $\begin{array}{c}-0.107 \\
(0.759)\end{array}$ \\
\hline Quite a bit & & $\begin{array}{c}-1.751^{* * *} \\
(0.000)\end{array}$ & & $\begin{array}{c}-0.810^{* * *} \\
(0.000)\end{array}$ & & $\begin{array}{c}-1.484^{* * *} \\
(0.000)\end{array}$ & & $\begin{array}{c}-0.584 \\
(0.088)\end{array}$ \\
\hline Extremely & & $\begin{array}{c}-1.951^{* * *} \\
(0.000)\end{array}$ & & $\begin{array}{c}-1.272^{\text {*** }} \\
(0.000)\end{array}$ & & $\begin{array}{c}-1.823^{* * *} \\
(0.000)\end{array}$ & & $\begin{array}{c}-0.796^{*} \\
(0.020)\end{array}$ \\
\hline Constant & $\begin{array}{c}2.491^{* * *} \\
(0.000)\end{array}$ & $\begin{array}{c}3.396^{* * *} \\
(0.000)\end{array}$ & $\begin{array}{c}1.864^{* * *} \\
(0.000)\end{array}$ & $\begin{array}{c}2.026^{* * *} \\
(0.000)\end{array}$ & $\begin{array}{c}1.818^{* * *} \\
(0.000)\end{array}$ & $\begin{array}{c}3.117^{* * *} \\
(0.000)\end{array}$ & $\begin{array}{c}1.097^{* * *} \\
(0.000)\end{array}$ & $\begin{array}{c}1.741^{* * *} \\
(0.000)\end{array}$ \\
\hline Observations & 686 & 686 & 636 & 627 & 650 & 650 & 660 & 660 \\
\hline Adjusted $R^{2}$ & 0.035 & 0.229 & -0.002 & 0.089 & 0.044 & 0.150 & 0.261 & 0.333 \\
\hline
\end{tabular}

$p$-values in parentheses

${ }^{*} p<0.05,{ }^{* *} p<0.01,{ }^{* * *} p<0.001$ 
Table A12: Experimental Effect on Inappropriate by Scenario, Extraction Hypothesis (H4)

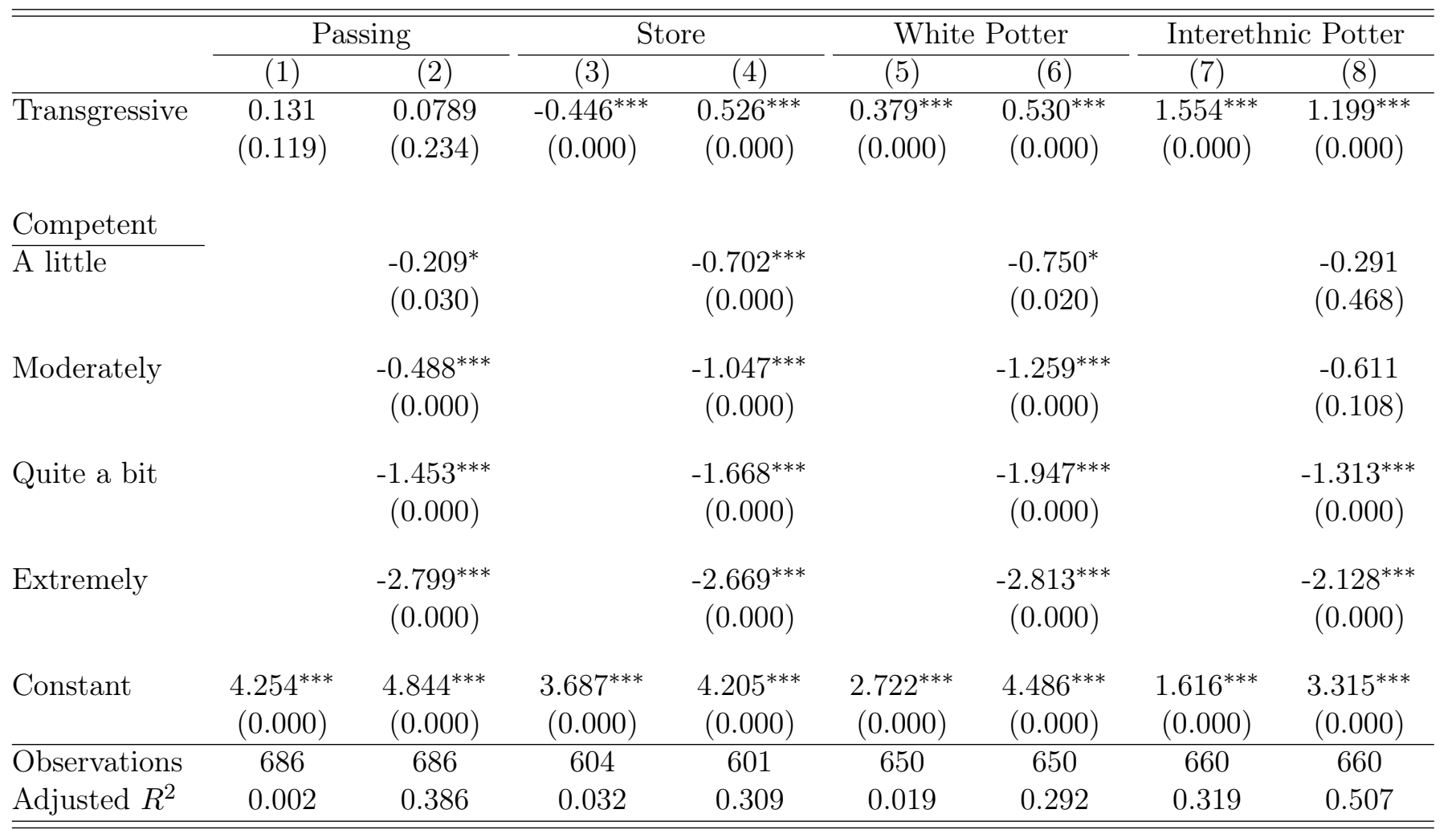

$p$-values in parentheses

${ }^{*} p<0.05,{ }^{* *} p<0.01,{ }^{* * *} p<0.001$ 


\section{Stimuli}

The following tables provide full descriptions of all the stimuli used in the experiment. 


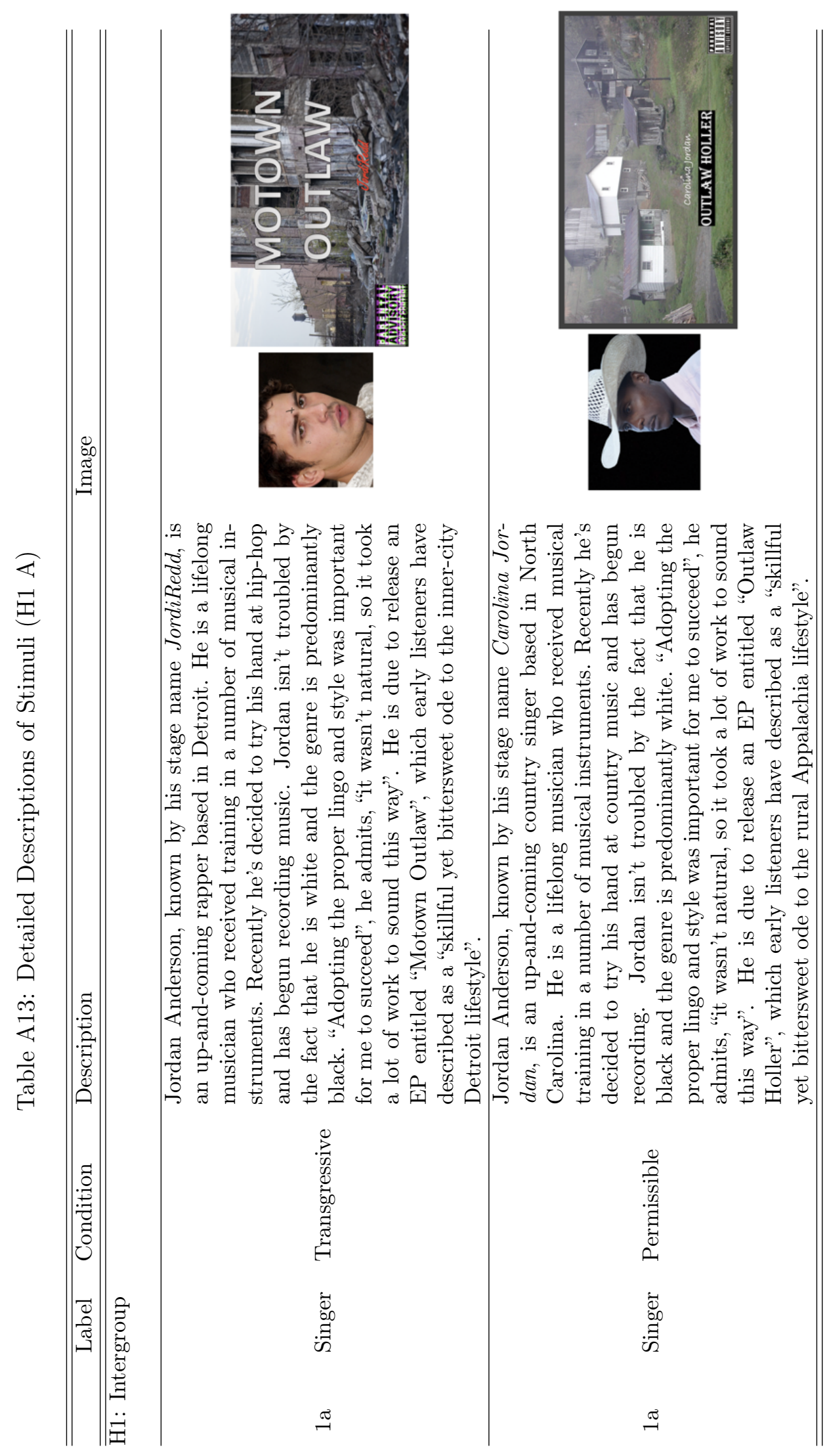




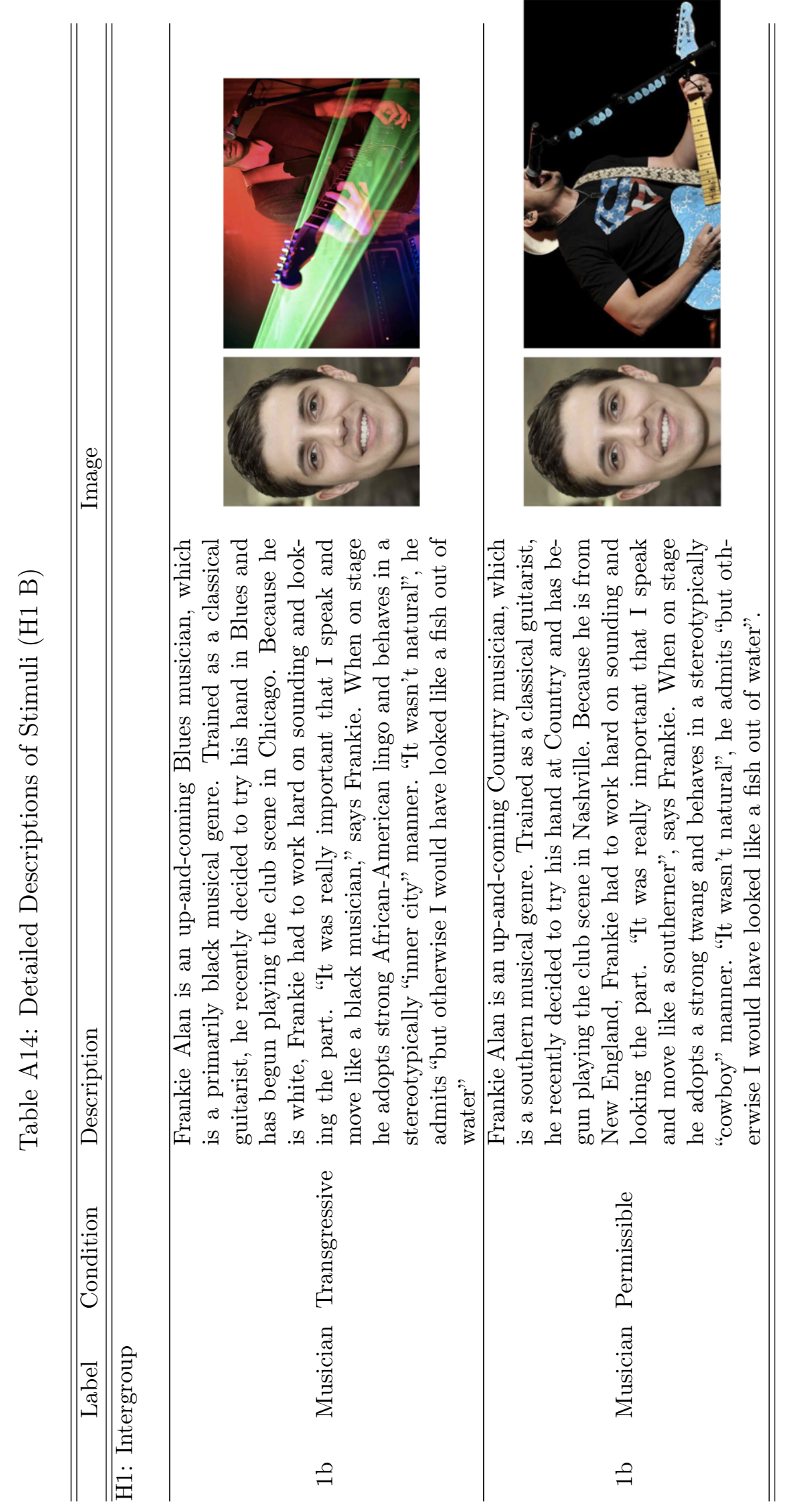




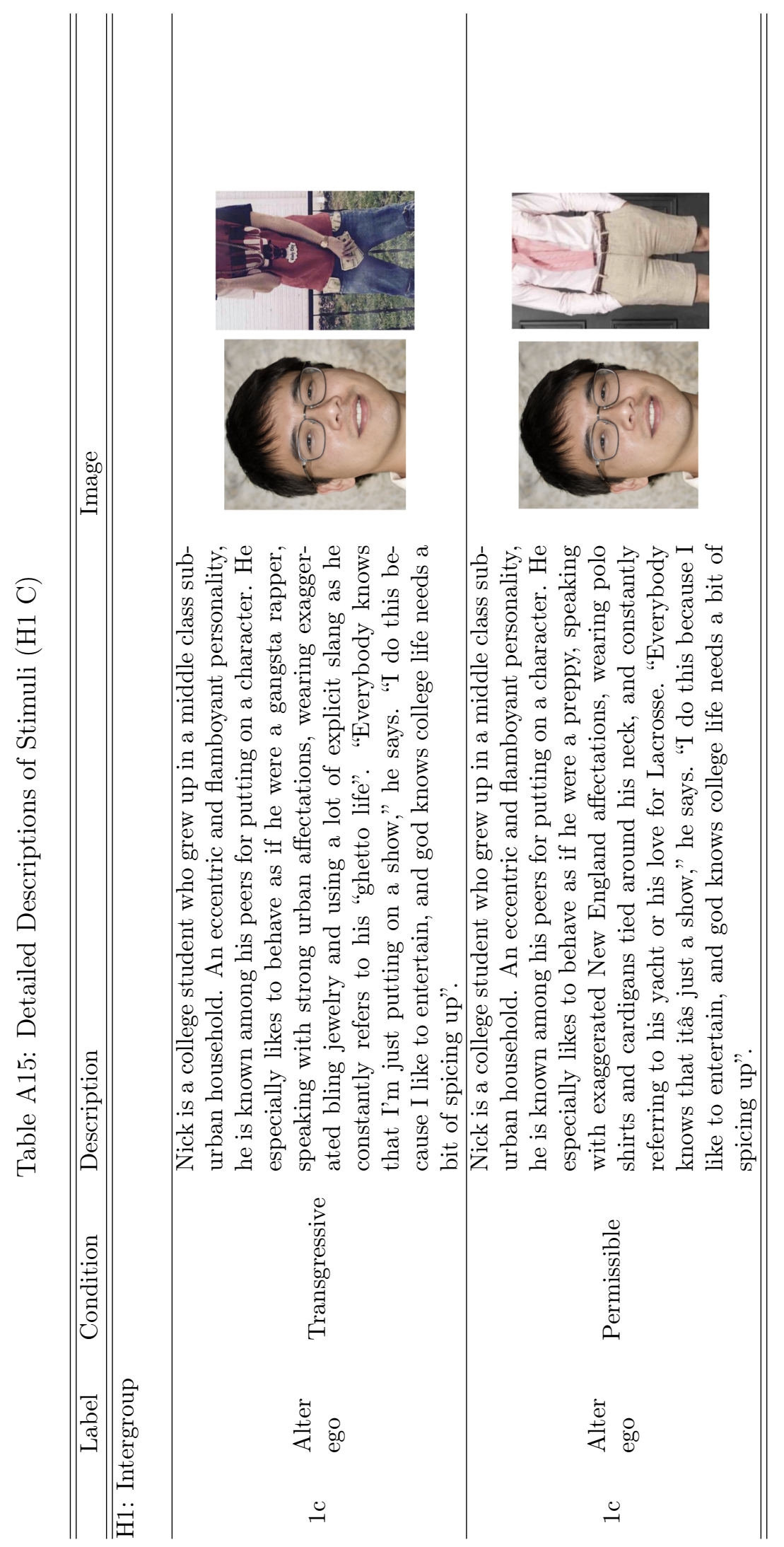




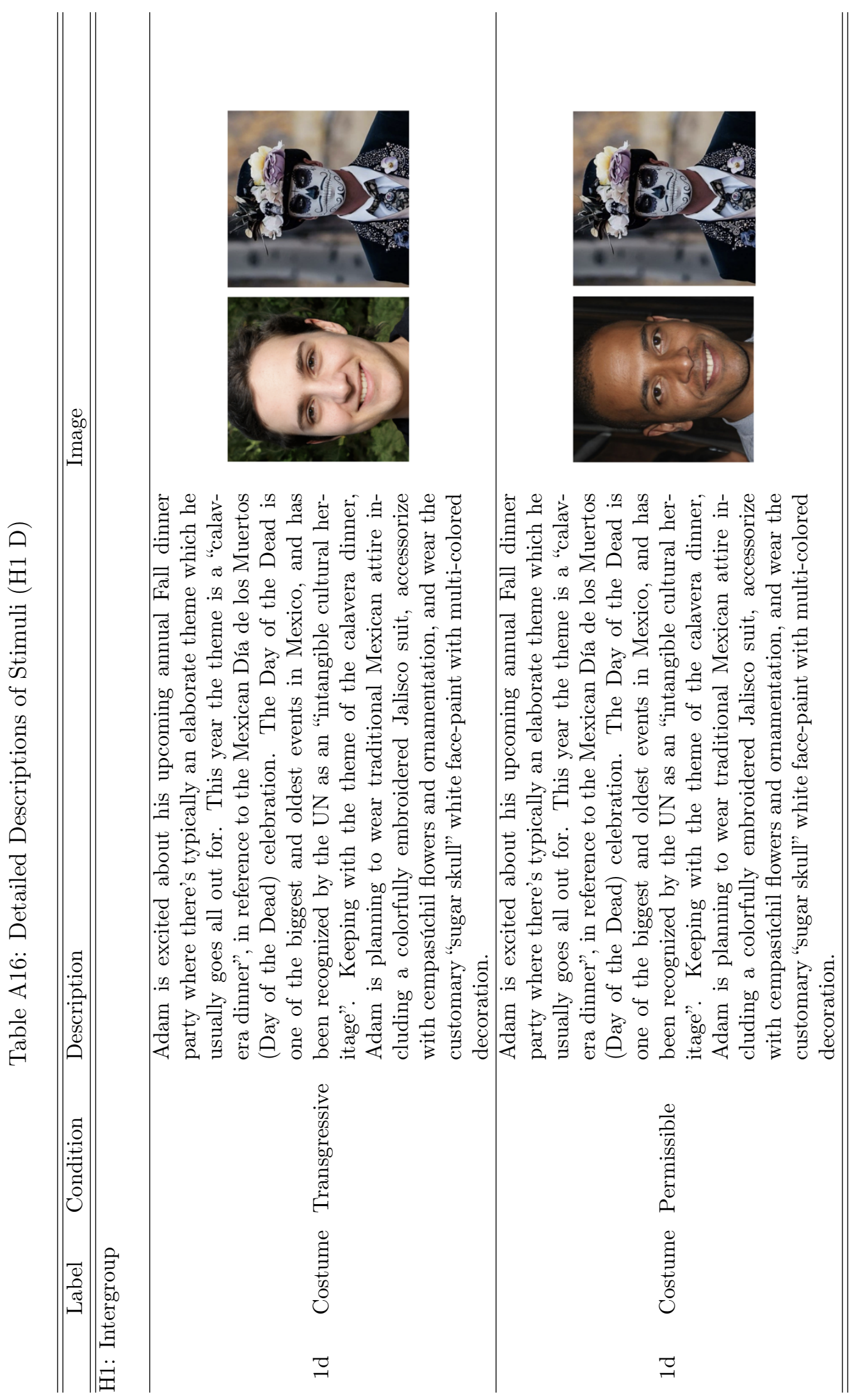




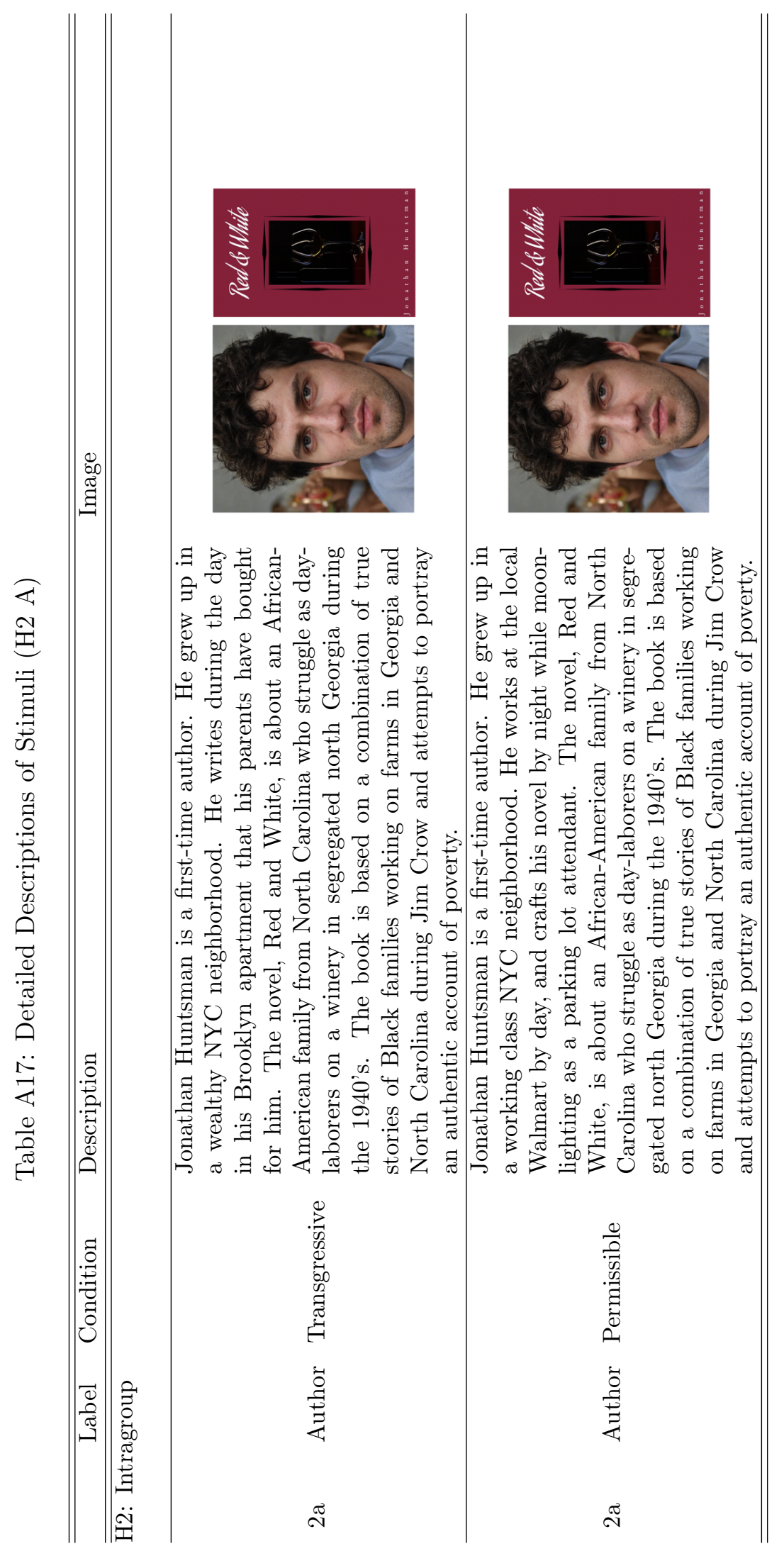




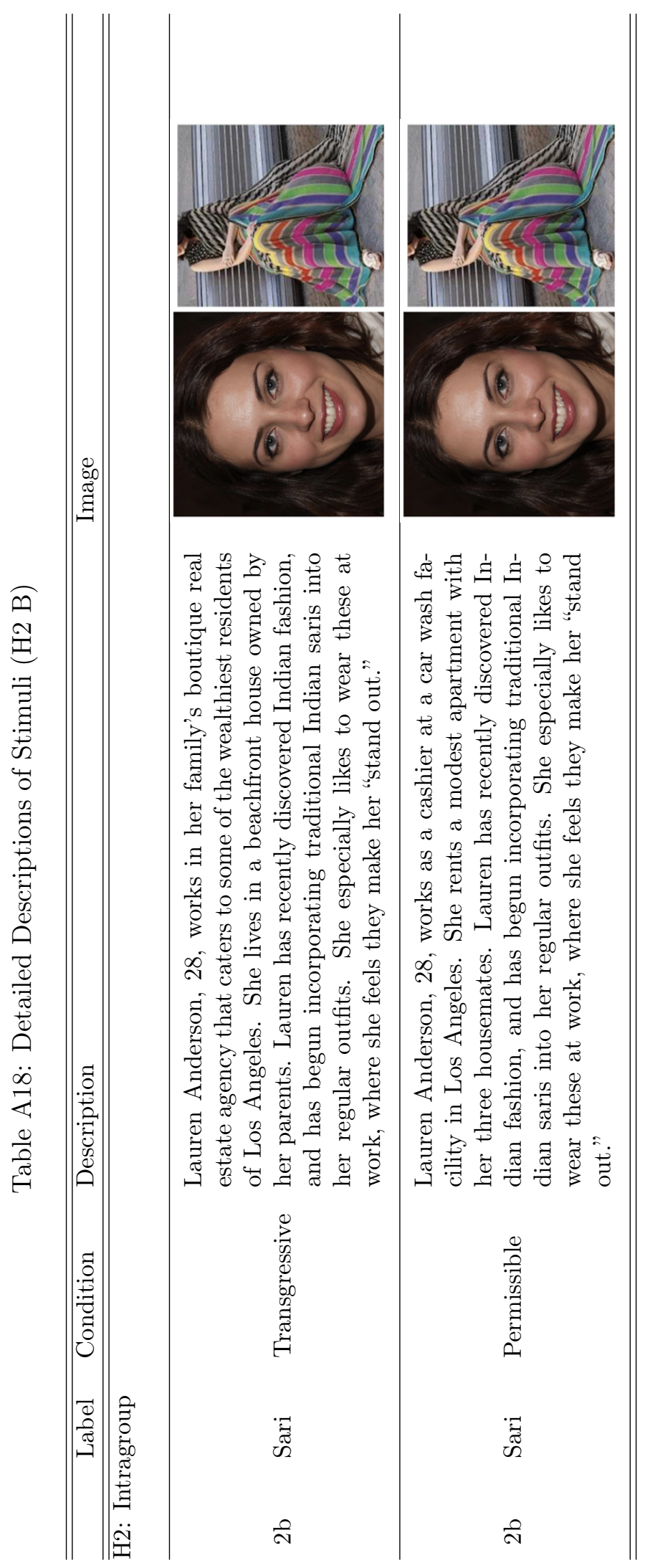




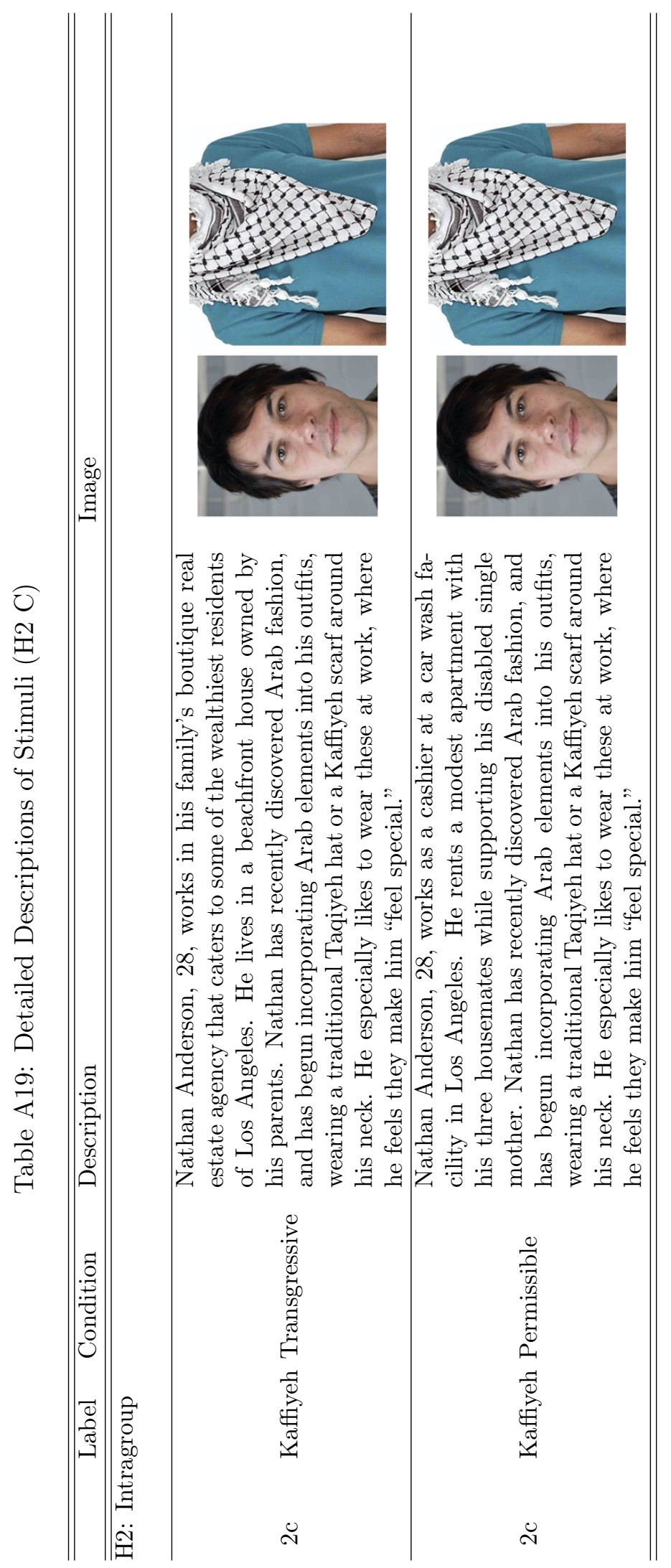









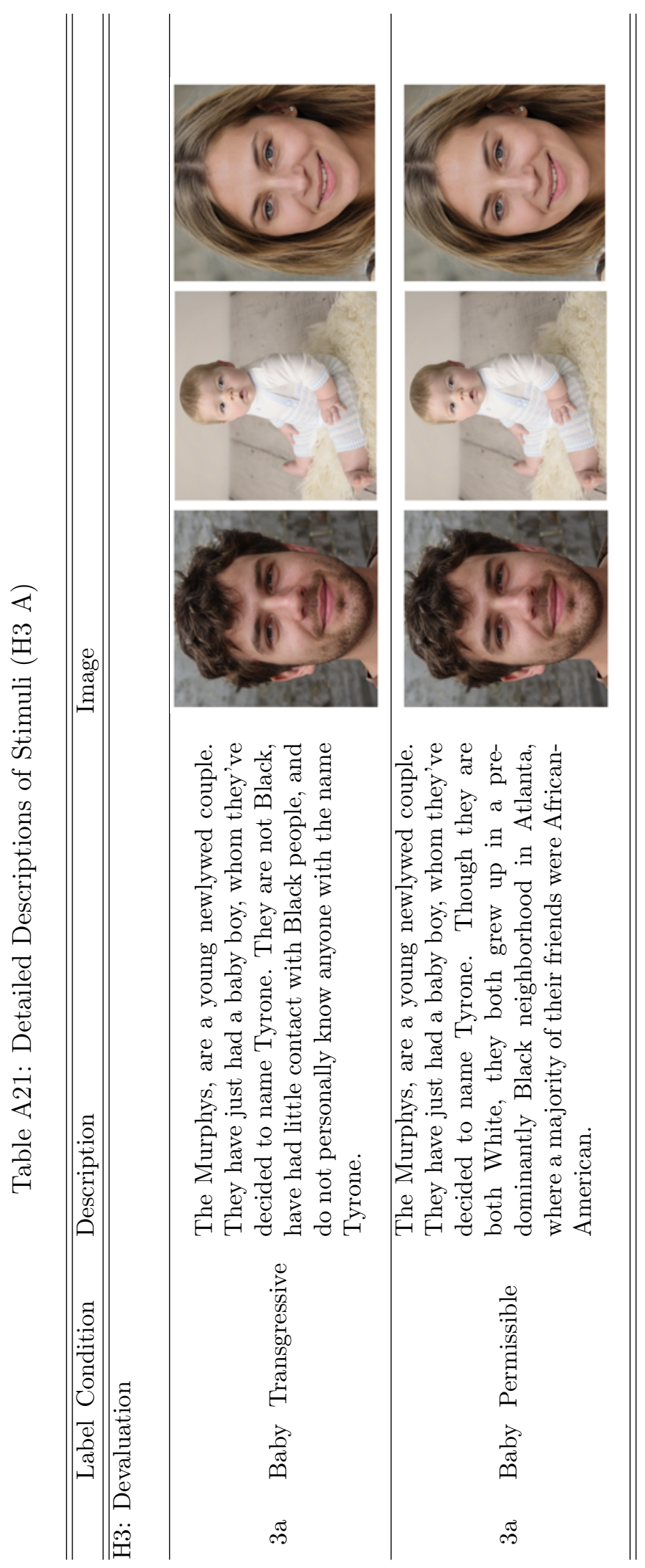




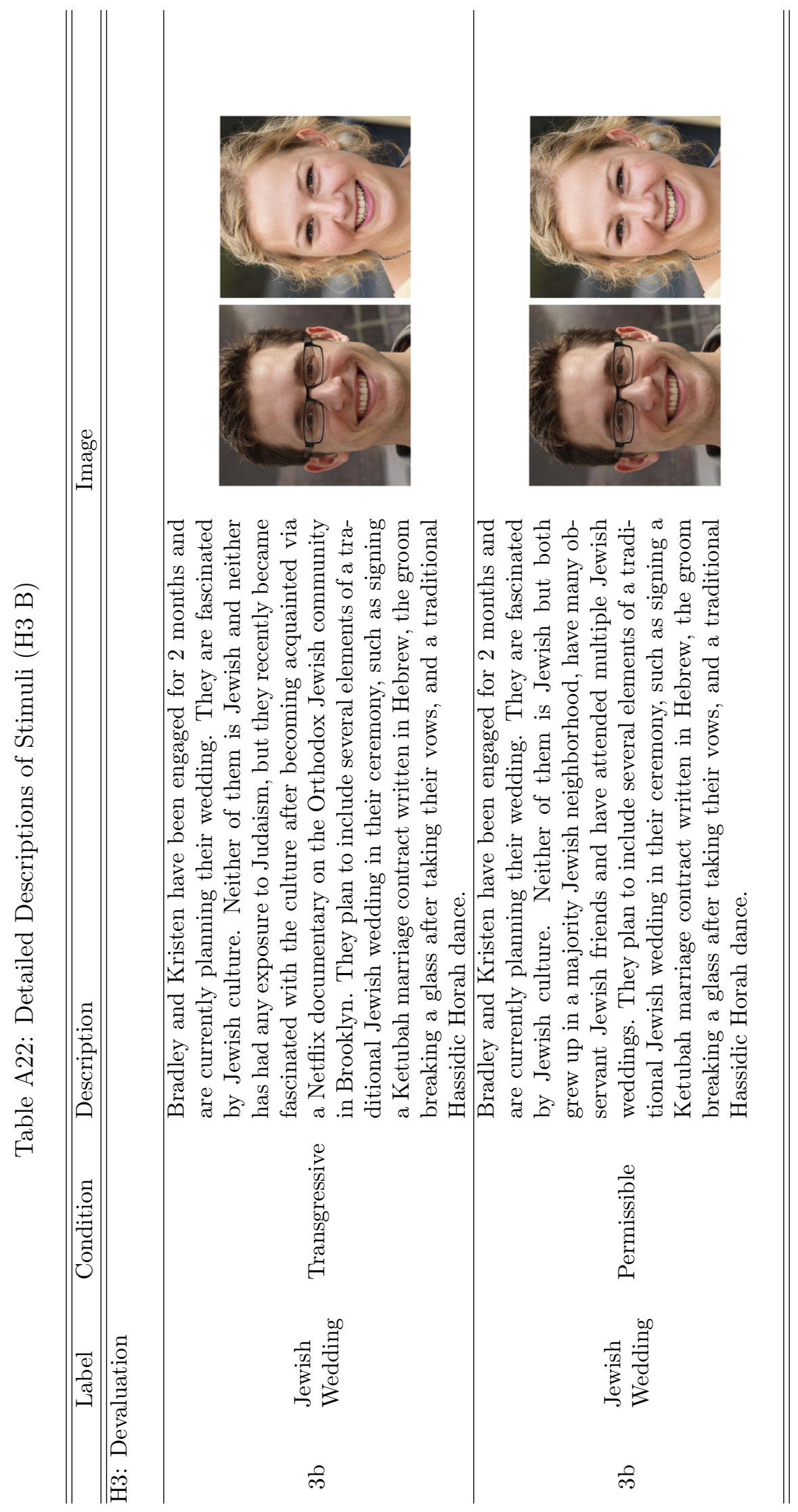




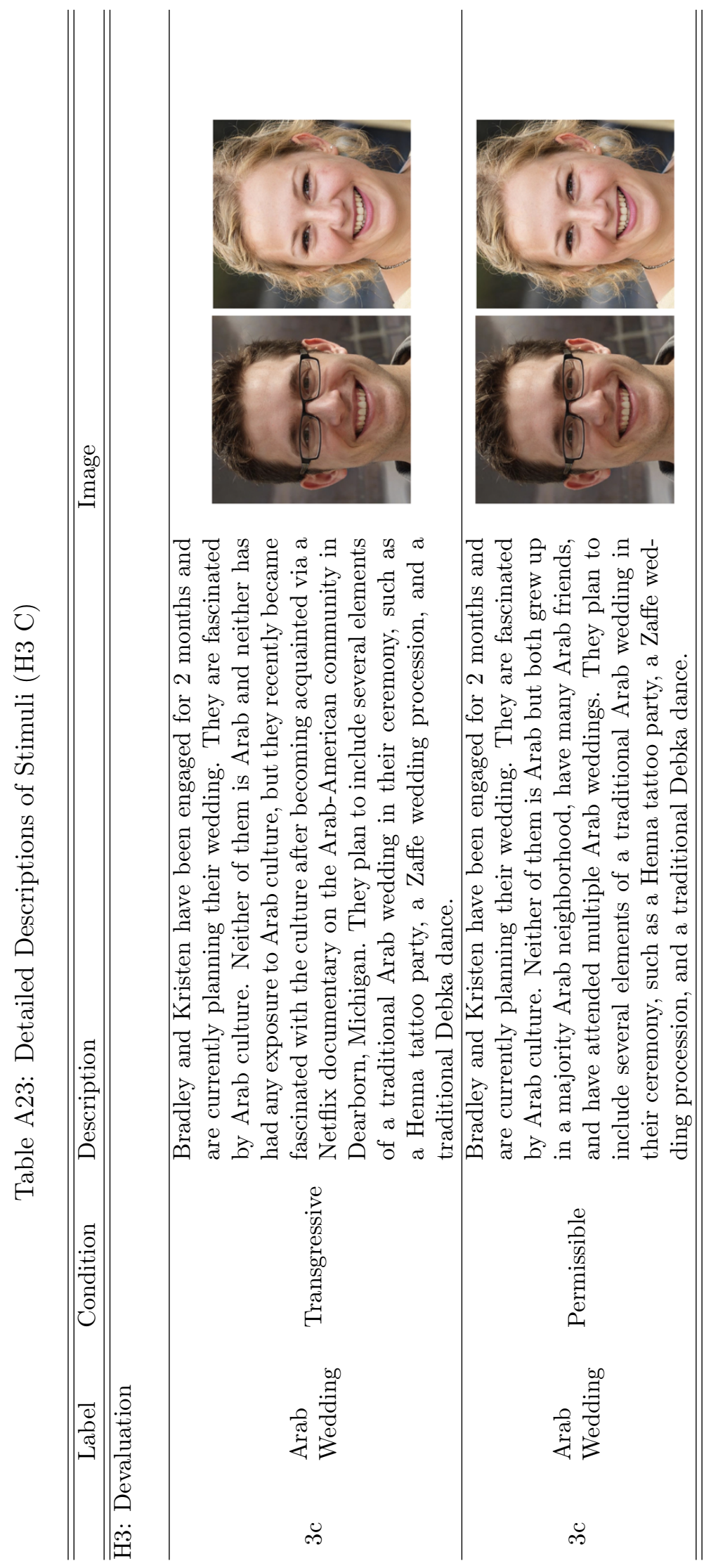




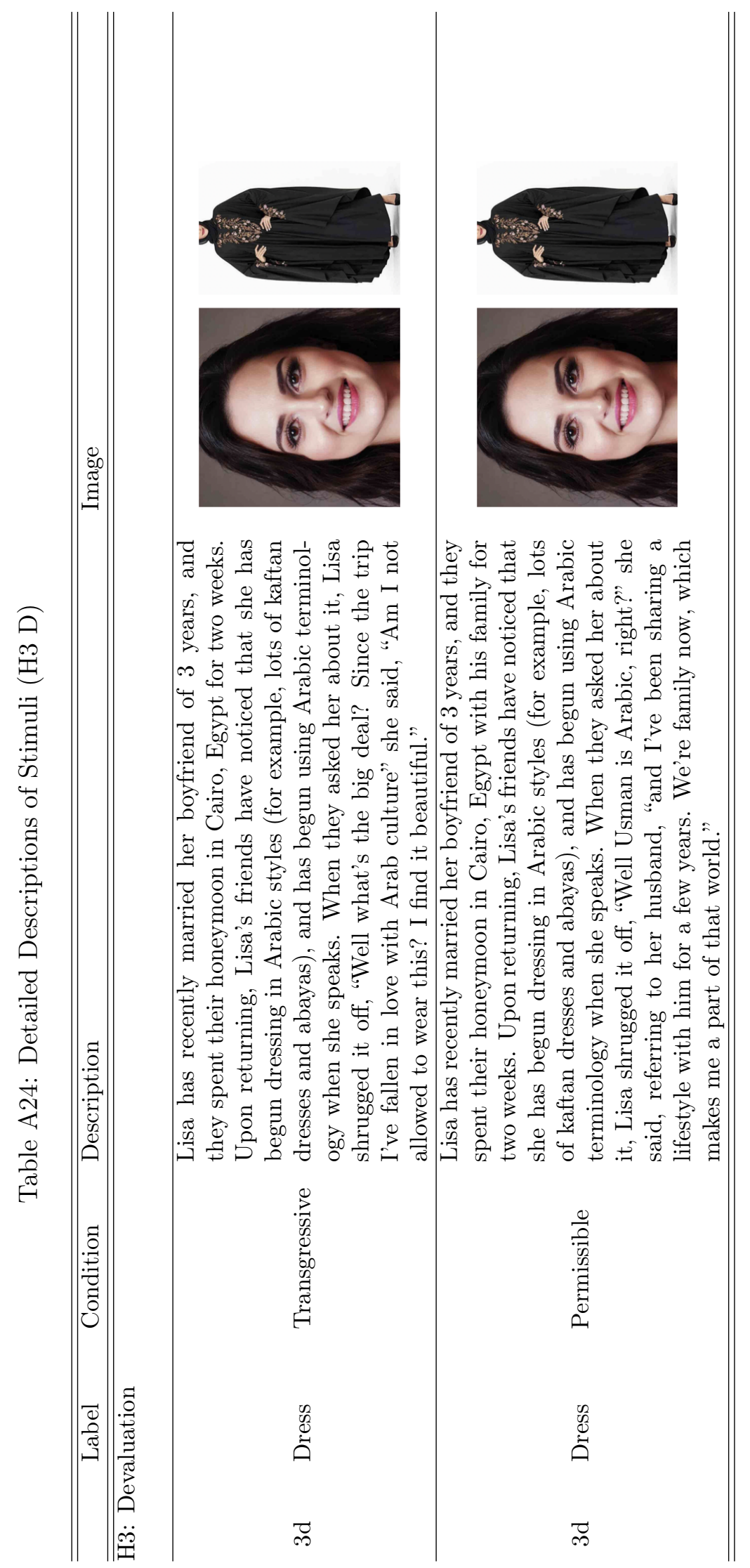




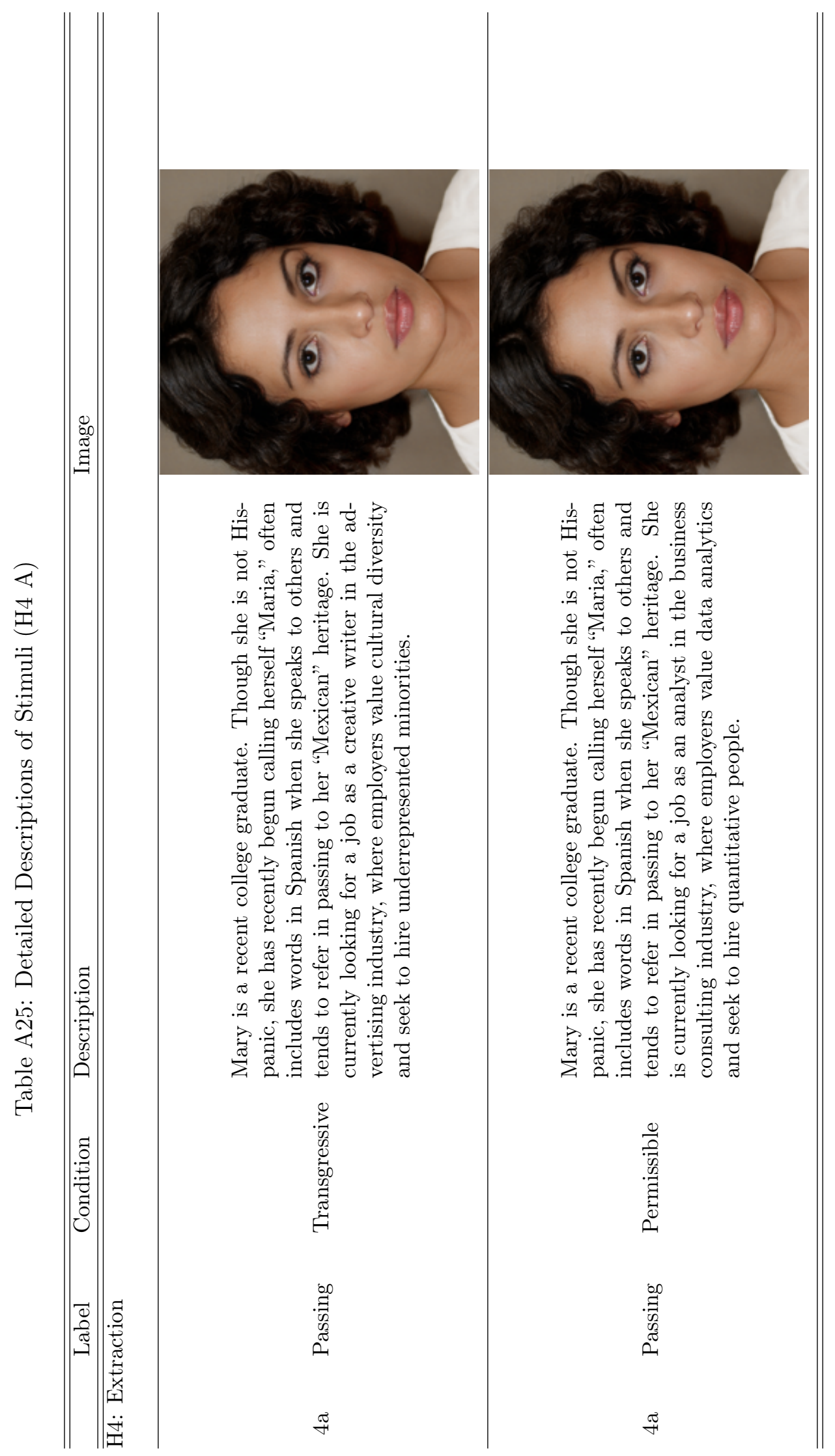




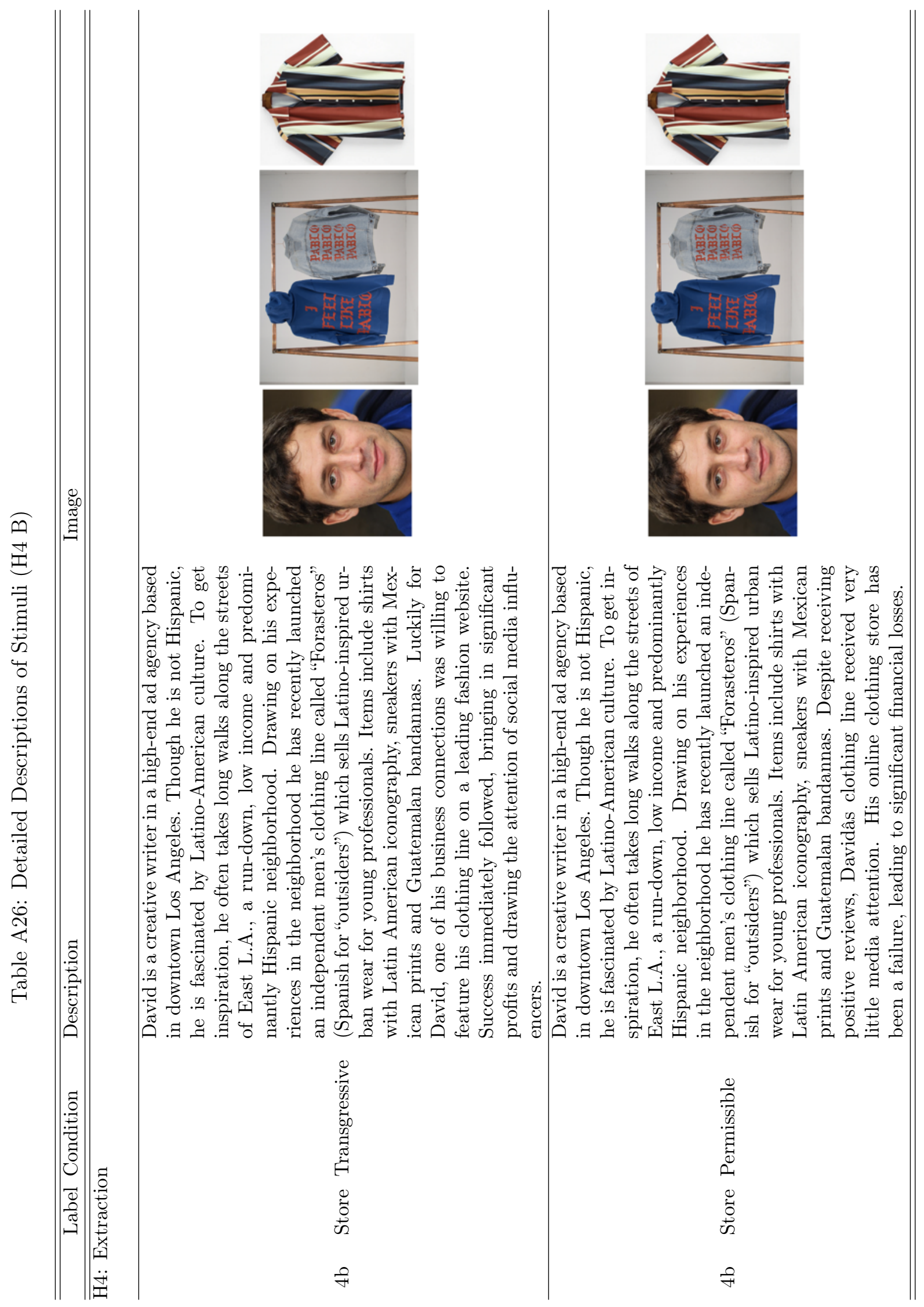




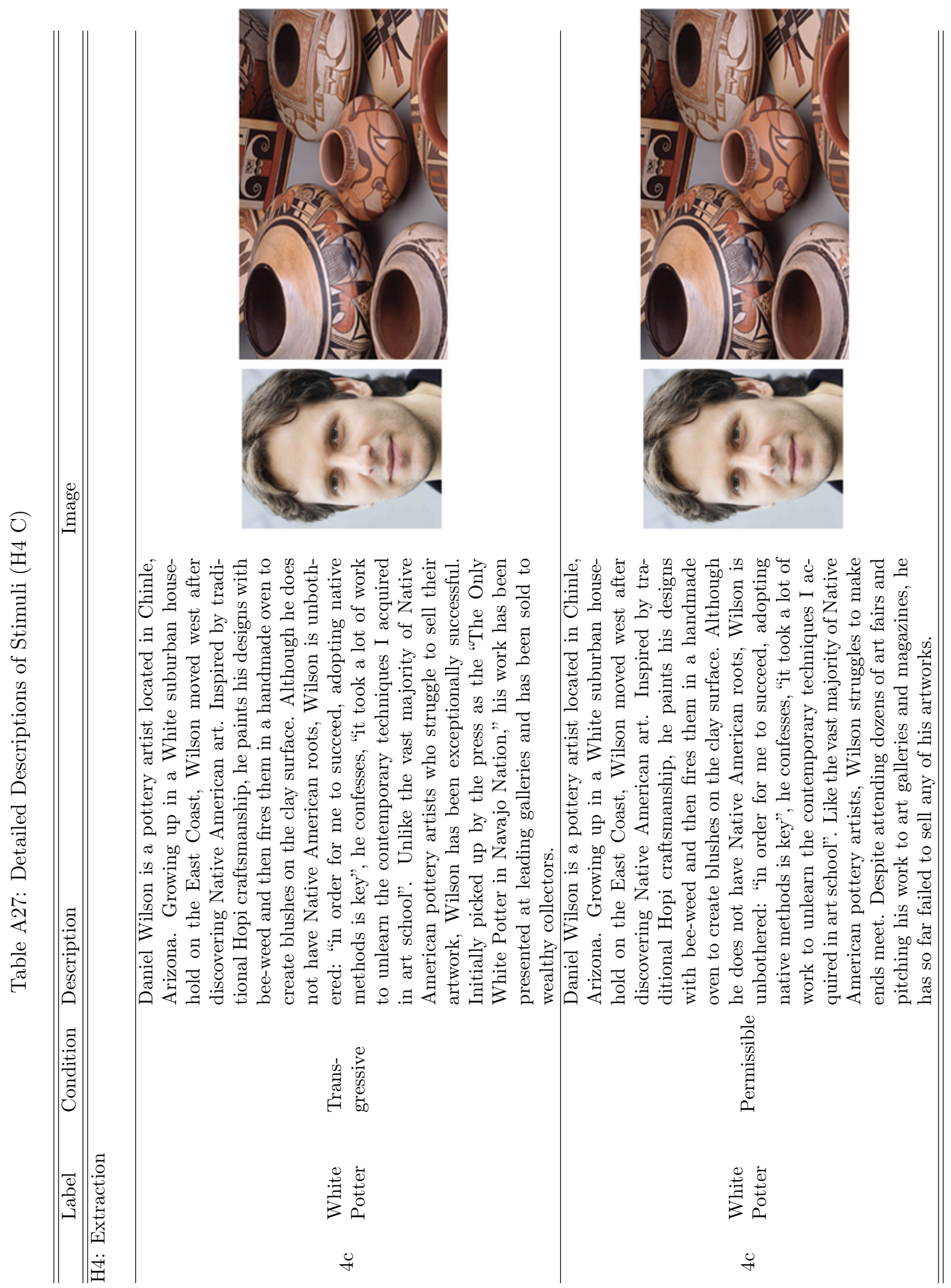




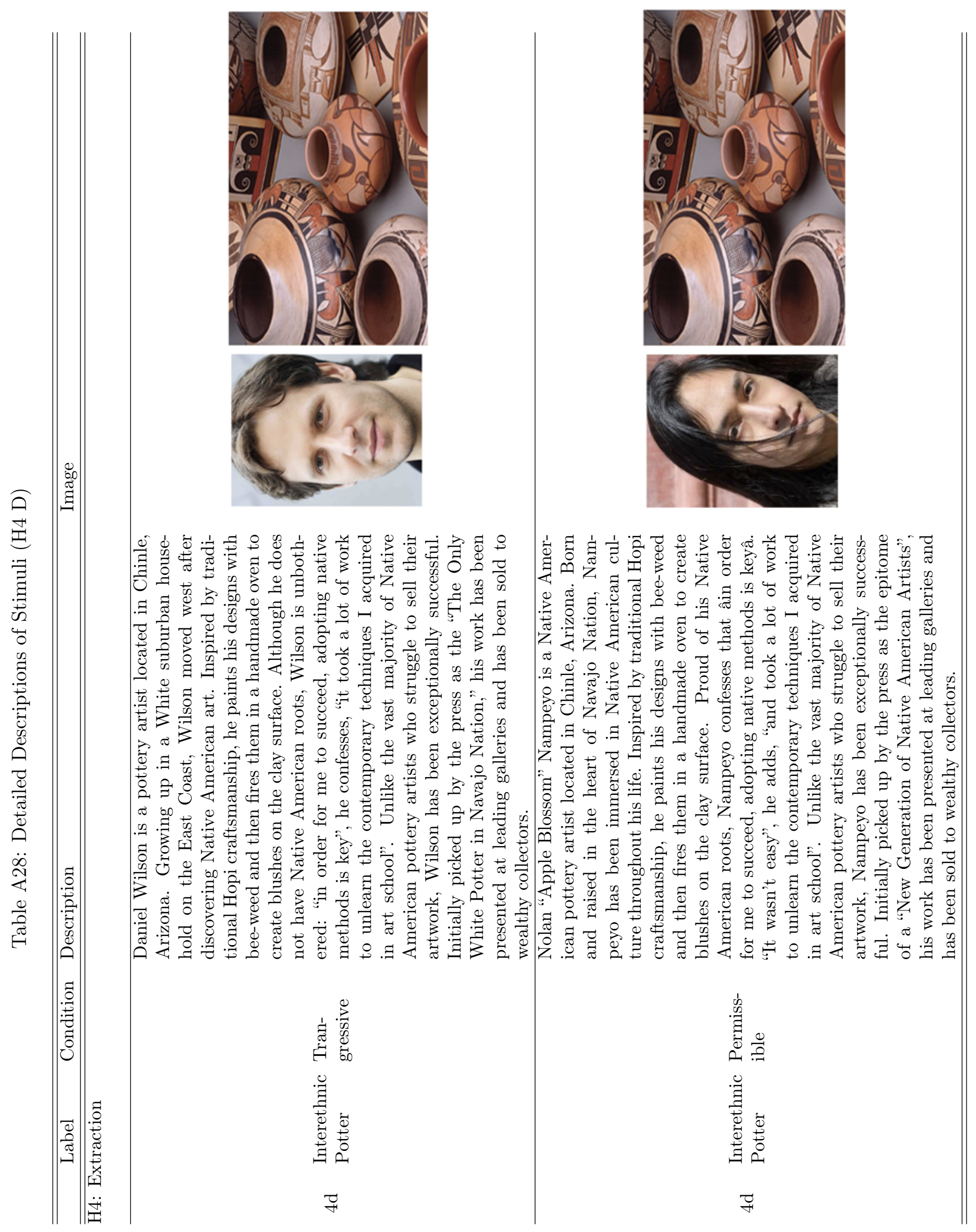

